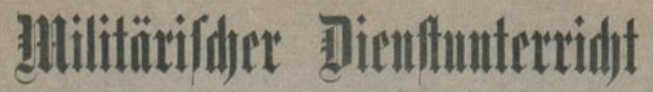

fïr

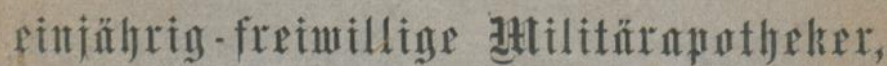

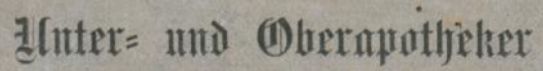
Des

- Itentrijen fieexes.

$\because$

Bearbeitet

yon

Dr. ม. Thüıtร,

Rintigl. Skerawotheter ber Rejerbe.

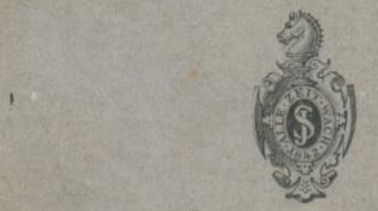

fierlitt.

Berlag von วัน lius Springer. 1889. 

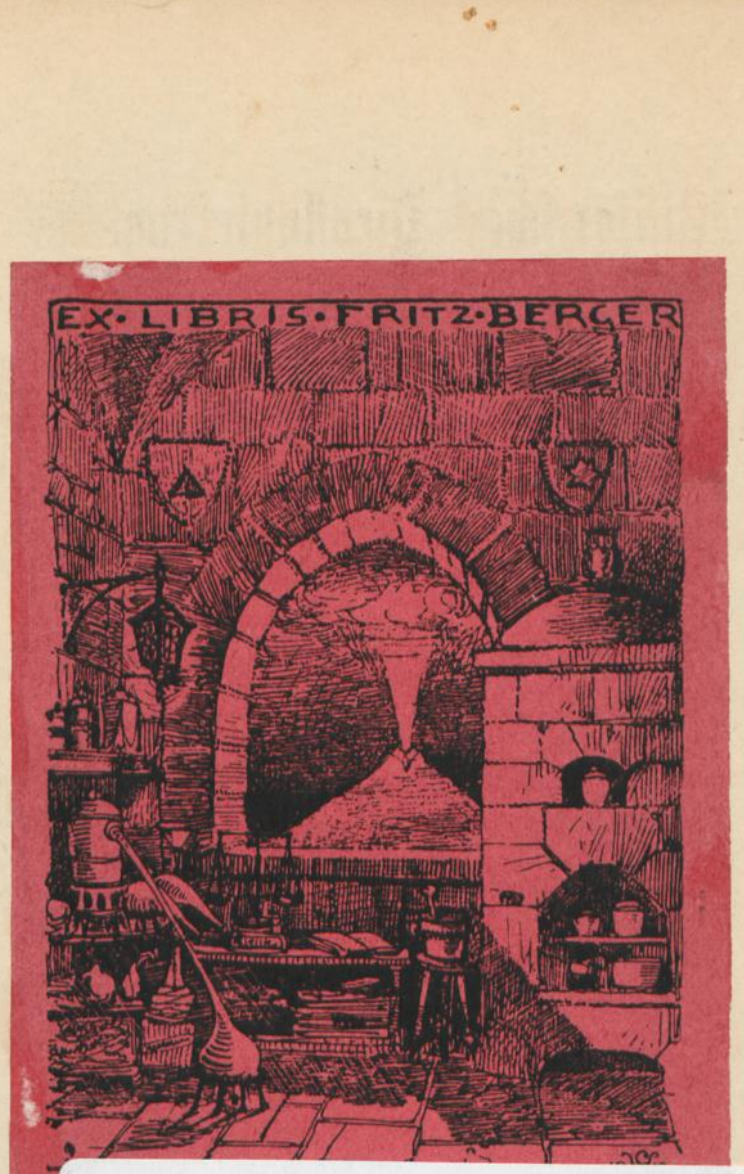

ULB Düsseldorf

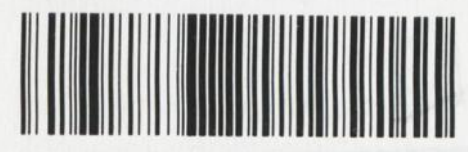

$+417981801$

16110

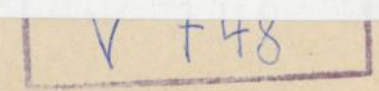

$160^{\circ}$ 


\section{Mnilitärifider Dienffuntertidut}

für

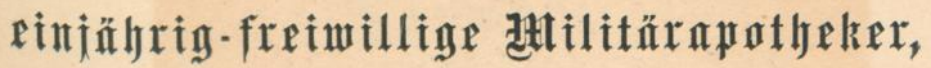

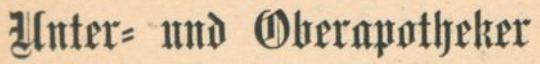

Des

10

Bearbeitet

bon

Dr. d. Thảhts,

Sönigl. Sberapothefer Der Referbe.

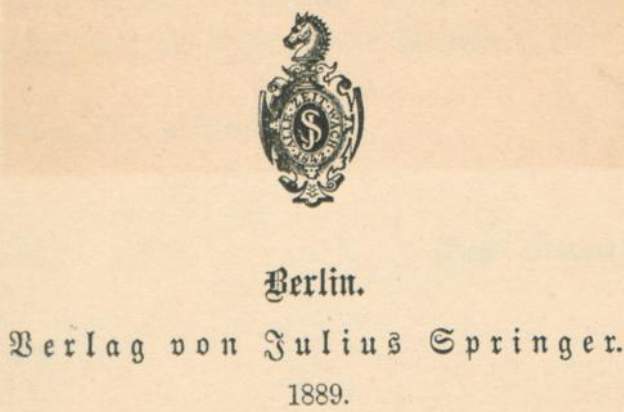

1889. 


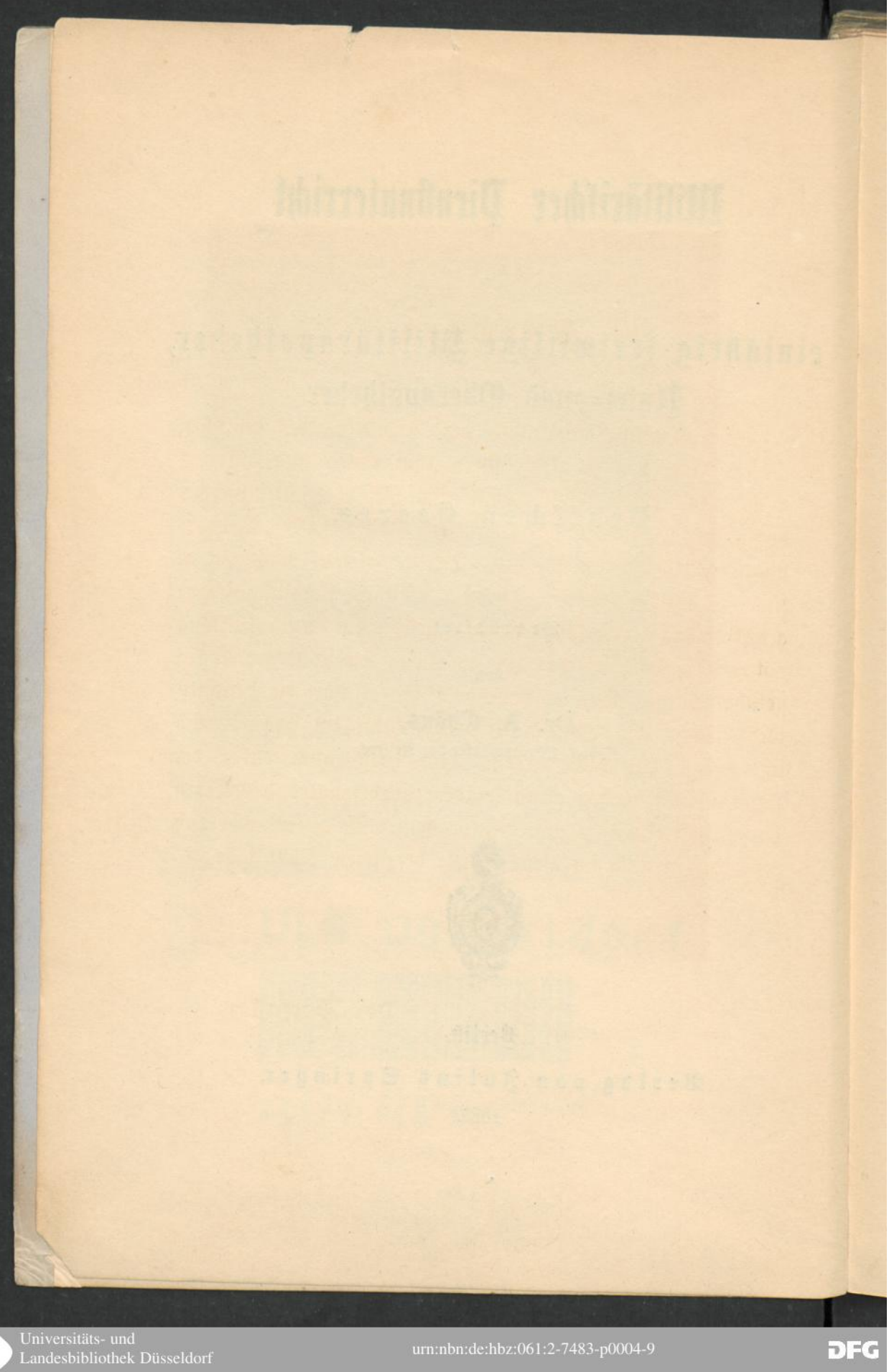




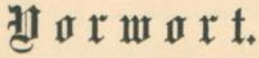

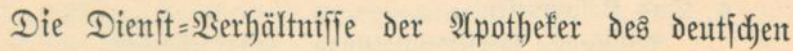
Şeeres find vor ca. 10 Sahreet zum erftent und bis jeb̧t

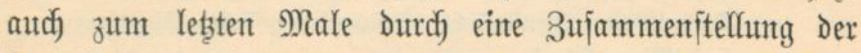
bez̧üglidchent Bejtimmungen bearbeitet wordent (Der "Mitlitär= pharmazent" von Dr. Büttger. Berlin 1879). Naturgemäß̊ find in Dem gentanten Beitraum viele Beftimmungent $\mathfrak{a} \mathfrak{b}=$

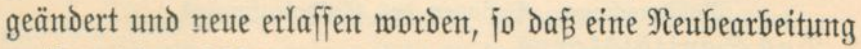
ntothwendig erjodient, was mir in einem Streitfall vor Ŝtugen

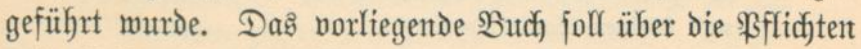

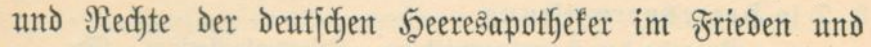
im Siriege গtuffidluf gebent. Sd, batte midid dabei der Unterftüţ̧uty mehrerer Sameraden, inşbejondere Des Şerrn

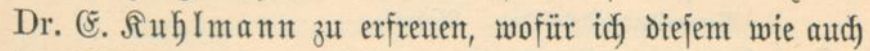
Den ïbrigen aud) an biejer Stelle herzlidjen Danf jage.

Berlin, im Dftober 1889.

\section{Der כerfafler.}




\section{वิ $\mathfrak{n} \mathfrak{l} \mathfrak{n} \mathfrak{l} \mathfrak{t}$.}

I. Angemeines.

Seite

1. Das eigentlidje feer. . . . . . . . . . . . 1

A. Das Erjaknefer . . . . . . . . . . . . . . . 1

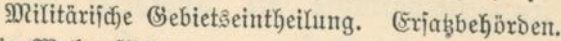

B. Die Wefrefficht

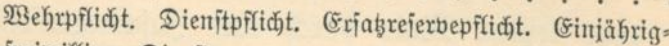
freinilliger Dienit.

C. Die Truppentheile

Baffengattungen. (Eintheilung. Bertheilung ber $\mathfrak{2}$ (rmees

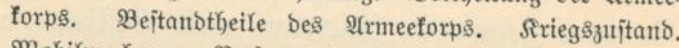
Mobilmad)ung. \$erionen des Soldatenitandes.

2. Das Santiätsłorps

Sanitäts̊perfonen. Ç̧argen. Itniform ber Sanitäts̊ofifiziere umb übrigen Sanitätsperjonten.

3. Die Seeresperwaltung .

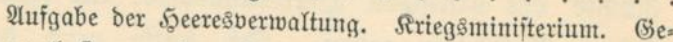
neral $=$ Sommando. Sntendantur. Sorpsarzt unb Sorps: apothefer. 2(rznei= uno $\mathfrak{B e r b a n b m i t t e l = \Re e j e r v e . ~ D e r t l i c h e ~}$ Berwaltungsbejöroen. Beante.

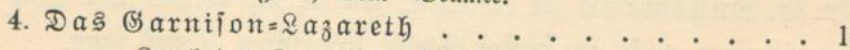

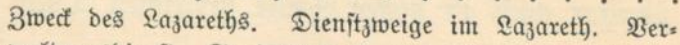

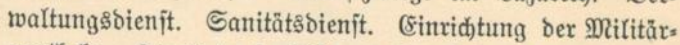
apothefe. Dienjt in berielben. Arznei=Empfänger.

\section{Die şecresapotgiefier.}

Die Fुeeresిapothefer munffen als 2(pothefer gedient haben. Eaufbafyn ber ફ̧eeres̊apothefer. 21pprobation $\mathfrak{B}$ ebingung Deకి Eintritts. Eintritt\$ 3 seit. Eintrittsorte. Eintritts= zeutgniffie. RegeIn für 2Gbfafjung bon Dieniticidriften. Ein=

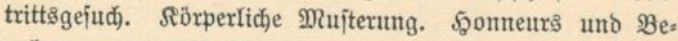
nehmen gegen $\mathfrak{B o r g e j e b ̧ t e . ~ S ̧ o n n e u r ~ b e r ~ D i f i z i z e r e , ~ b e r ~}$ übrigen. 
1. Der einjäbrigefreiwirtige 9 Tiritärapotgeter. Seite

Uniform. Intritt uno Meloung. Bereibigung. E̋i๖.

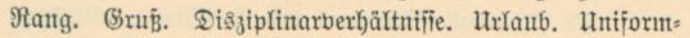
zwang. Gejääitsbetrieb in Der Difpenifiranjtalt. M3ort= laut einer Meloung. Bejdaffing ber $\mathfrak{A}$ rzneien. Bejtells

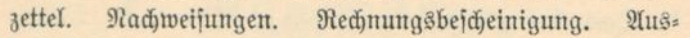

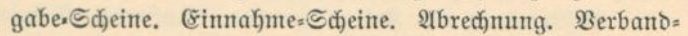
mittel. Irinfwaifier-lnterjudungen. Unterrid)t. Prüjung Beförberung. Afbmeloung. Interapotheferuniform. Walb=

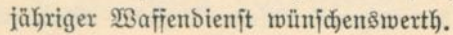

2. Der Unterapothefer . . . . . . . . . . . .

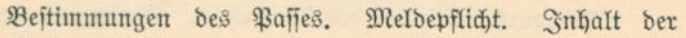
Meloung. Abmeloung vor ber Abreife. Dienitpflidft. Sontrollberjammlungen. Diş̧iplinarberbältnifïe. Burücts fitellung hinter bie leḅte Эahresflafife. Einfoummen im ซalle ber (Einziehung. Beförderungşantrag. Bejörberung.

3. Der Dberapothefer .............. . . . .

Rangitellung. Berjonalbogen. Ranglifte. Melbung beim Bejirfss:Sommandeur. Uniform. Sintrollberjammlung. Diss ziplinaritrafent. Bejchwerbe $2 B e g$ unt Behandlung. Bejuche. Bejontere Meldungen. Difiziersverjaumulungen. Difizizier=

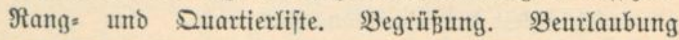
unঠ $\mathfrak{A} u$ ș Deutidider Difizizierverein.

4. Der Feldapotheler . . . . . . . . . . 7t

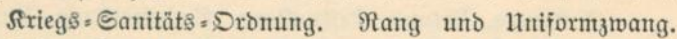

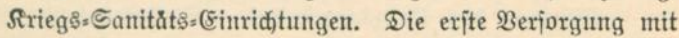

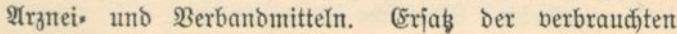
Mittel. Begrifi unb ðorm ber Requifition. Etappen.

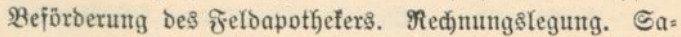
nitätşbienit auf bem Siampiplał̧. Iruppenberbanoplał̧.

A. Das Santïäts= Detadjement . . . . . . . . . . 74

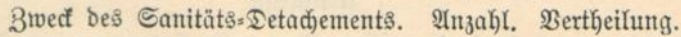
Berfonal. Material. Diş̧iplinarberhältnifife. Bertretung. Drt bes 5ुauptberbanoplą̧es. Iransport ber $\mathfrak{B}$ eriwunbetert nad) Demfelben. Dienit auf bem Berbantoplab̧. Die 3 शtb. theilungen befielben. Ausgabe ber ḩeils und $\mathfrak{B e r b a n t}=$ mittel. (Exałab Derjelben. Abred)unng. 
B. Das Feld Ia 3 areth 3twett bes frelolazareths. 2(nzahi und itmfang. \$erional

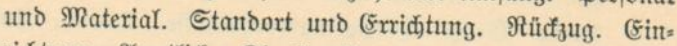

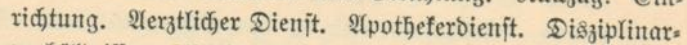

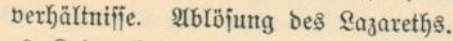

C. Das Rriegsla $a_{3}$ areth .

Beftimmung bes Siriegslazareths. Perional. Dişziplinar: verbäItnififfe.

D. Das $\& \mathfrak{a}_{3}$ areth $=$ Rejerve $=$ Depot .

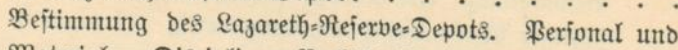
Material. Dişziplinar $=$ Berhältnifife. Berwentoung unt Etandort. 2Ubgabe des Materials. Ergänzung der $\mathfrak{B e}=$

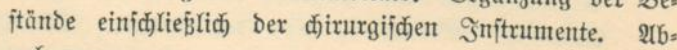
rectgmung.

E. Das immobile Güter=Depot an ciner Sammelitation . 3weff bes Depots. \$erional.

F. Das Rejervela $a_{3}$ areth

Begriff Des Rejervelazareths. Bejonbere Bejtimmungen.

Bebüriniz zur Injtellung eintes 2(pothefers.

G. Das Feitungslazareth .

Begriff Des ₹ejtungs[azareths. Bejondere Beftimmungen. Ihätigfeit bes Garnifonarates.

5. Der Stabsapotyefer.

2Birfungs̊freis ber Stabsapothefer. Einberufung und 2 (nt= ftellung. Ģeld = Gebührniffe. Rangverbältniffe. Sbere Snitanzen. MrIaub. Erfranfung. Berheirathung. Uniform. Pflichten des Siorpsapothefers. Stellvertretende Sorps:

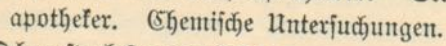

6. Der Dberitabsapotbefer WSirfungsfreis. Mebizinalabtheilung. Pflichten ses ober. itabsaapothefers. Gebülhrniffe. Rang.

\section{Antịang.}

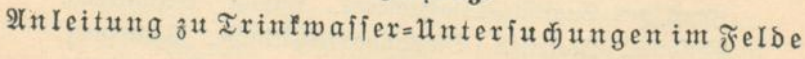




\section{Allgemeitites.}

\section{Zuts einentlidje fjerr.}

\section{A. Dả Criatżejett.}

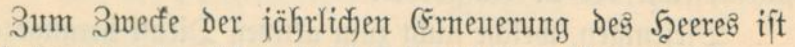

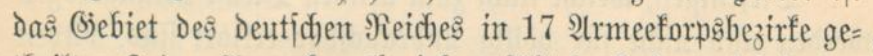

Militär• (B) biety:= eintbeilung theilt. Seder $\mathfrak{A}$ (rmeeforpąbezirf zerfüllt in Infanteriebrigade=

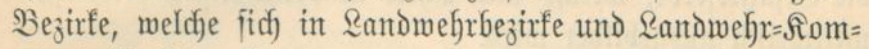
pagnie $=$ Bejuirfe gliedern.

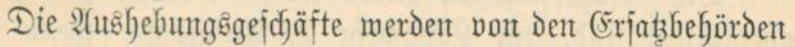
bejorgt. Dạ fint:

a) Errałbefördent der Minifterial= Snjtanz;

b) Erjaţbehörden Der 3. Injtanz, beftehent aus Dem (Shef Eriab= behörben eines $\mathfrak{A}$ (rmeeforps und dem Shef der Frovinzial= oder Rautdesvermaltungăbeförde;

c) Dber=Ěrjałfommififion, bejtehent aus dem Snfanterie= brigade = Sommandeur oder Randmefre $=$ Injpecteur und einem Cinil=sommifif ar;

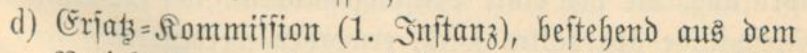

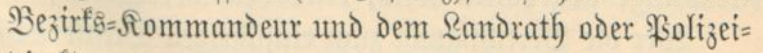
Direftor.

\section{B. Die $\mathfrak{B}$ Gefrepflidft.}

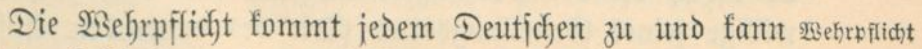
nidjt durd) Stel(fertretung geleiftet werden. Sie Dauert vom 
I. 2IIgemeintes.

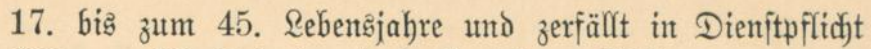
(bi⿱ zum 39. Sahre) und in Randjturmpflidyt.

Dienituflidit Die Dienftpflidyt unterjodeidet fid in aftive Dienitpfliddt

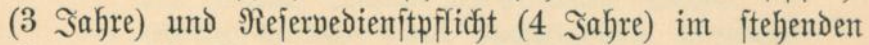
Seeere, und in Randwebroienfitpflidat und Erjabrejerbe= pflidjt.

Der Rantomebroienit zerfüllt in żmei Nufgebote. Da:

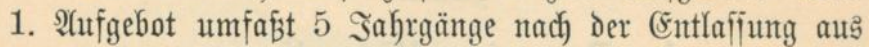
Dem ftehendent Sceere, dą 2. NUfgebot die übrigen bis zum 31. Närz des 39. Rebenșjahres. Dant beginnt die Rand=

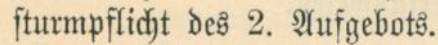

Eriak: rejerbepflidit

Diejentigen, weldhe nidyt zum aftiven Dientt herangezogen werden und nidyt für völlig unbraudb) die Ẽrjabrejerve, meldye zum Iheil in Dreimaligen Hebungen

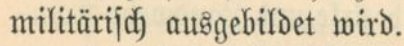

Die Ërjabrejerviften treten nad) 12 Jahren, went fie

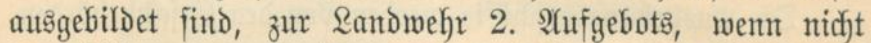

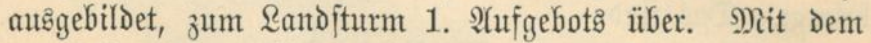
39. Rebentajahre gebören alle zum Randjturm 2. SAfgebots.

Einjăhrig. freim.

Dienit

Sunge Reute, die ein beftimmtes Maá̉ von Billoung bejiben und fich jelbjt befleiden, betöftigen und ausarijten, haben die Beredytigung, nur ein Эahr aftiv und 6 Safre in Der Rejerne zu Dienent. Die Bered)tigung fant Durd) ein

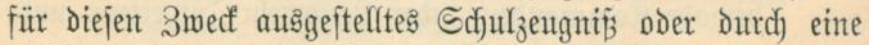

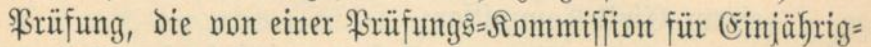
Jreimillige abgefalten wird, ermorben werdent.

SBer bie Beredftigung zum einjäbrigen Dienit nadjjudjen

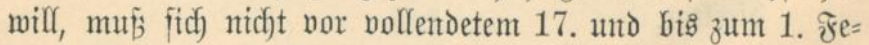

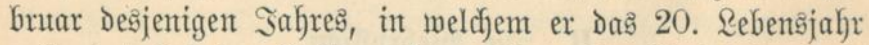

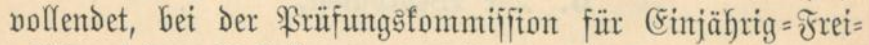
willige jeines (3ieftellunģortes (Drt Des Dauternden Alufent= baltes [Refre, Rehramitalt]) jodriftlich meldent. 
Der Meldung ijt beizufügent:

a) ein Sieburtåzengní; ;

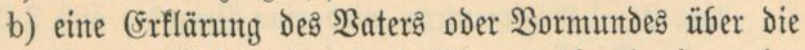
Bereitwilligfeit, Den Freimilligen während einer ein=

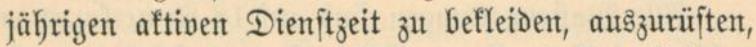

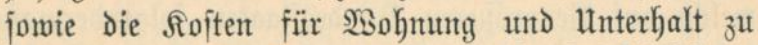
übernebmen. Die ₹ähigfeit Kierzu ijt obrigfeitlidy zut bejdjeinigen;

c) ein Unbejđoltentheitszzengnif́, Då für 3öglinge von höheren Sdullen nom Direftor Der Rebranjtalt, jonit von Der $\mathfrak{F o l i z e i o b r i g f e i t ~ o d e r ~ v o r g e j e b s t e n ~ D i e n j t b e h o ̈ r d e ~}$ ausิzu|itellen ijt;

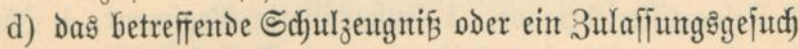
zur $\mathfrak{\text { Prüfuntg. }}$

Sit alles in Dromung, fo befommt Der Dienftpflidutige

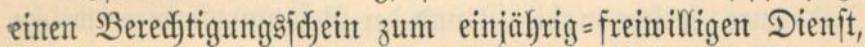

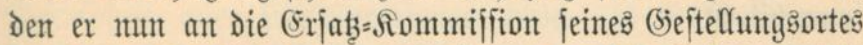

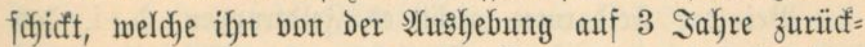

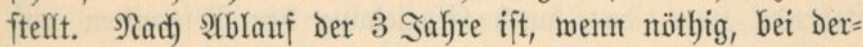
felben fommiffion eine abermalige Surüffitellung zu bean= tragen.

\section{Die Truppentheile.}

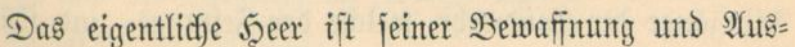
billoutg nach in $2 \mathfrak{S a f f e n g a t t u n g e n ~ g e t h e i l t ; ~ n a ̈ m l i d ) : ~ \Im n t = ~}$

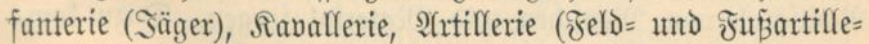
rie), જioniere (Eijenbahntruppen) und Irain.

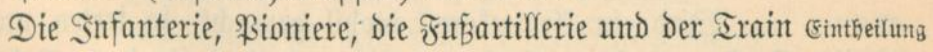
gliedern fidy in Bataillone und Siompagnien, Die Ravallerie in Eระfabrons und die Feld $=$ Artillerie in Âbtheilungen und Batterient.

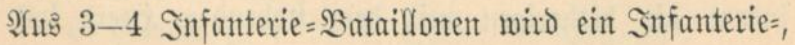




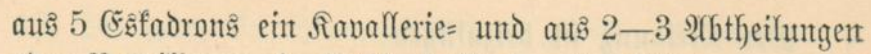

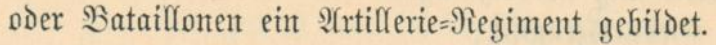

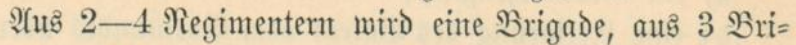
gaden eine Dinifiton zujanmentgejebt.

Bertbeilumg

ber

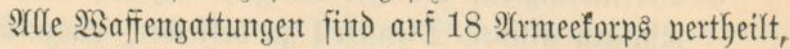
अrmeeforw meldje auf die gröberen Bundesftaatent folgentermafient ent=

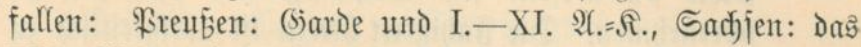
XII., Würtemberg: daร XIII., Băden: Das XIV., Bayent:

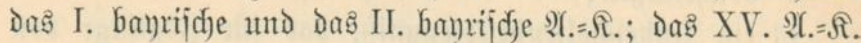
wird auร Iruppen verjdjedenten Staaten zujammentgejebt und fteht in Eljafi=?othringent.

Beitant. theile eines 2rmeeforps

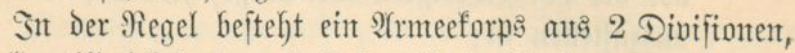

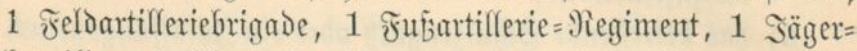
bataiflon, 1 Fionierbataiflon und 1 Irainbataiflon.

Siriegs: suitant

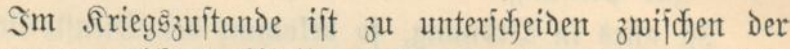
Jeldarmee, weldje mobil ijt und der Bejałungsarmee, weldje immobil it.

Bei der Feldarmee gieft eร Feldtruppent, D. i. Daร

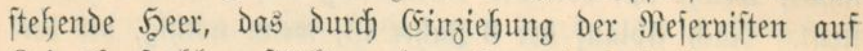

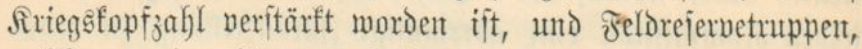
meldye aนเ den jüngeren Sabrgäntgen der Randwebr gebildet werdent (Rejerve=Divifitont).

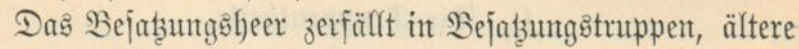
Sahrgänge Der Randwehr, weldje Den Feftung $=$ und Süften=

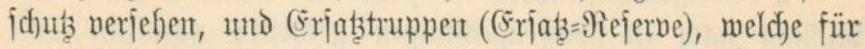
Nadjidjub von Mienjuden und Material jorgen. $D a z$ fom $=$

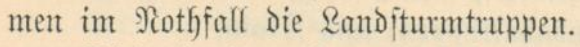

Mobil. mad)ung

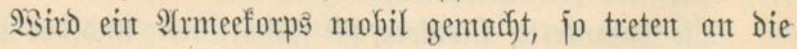
Etelle des Sienteral = א̃ommantos, D. i. Derjentigen Behörde, melá)e an der Spizze eintes Strmeeforps fteht, und an Stelle Der Infanteriebrigade "ftelluertretende" Derartige Bebürden, indem die bisherigen mit dem ftefenten Şeere int? Feld 
rïcfen. Die abrïcfenden Iruppen laffen entweder eintent be= ftehenten Imppentheil als Erjatztuppe jutüf (Navallerie, Pioniere) oder bildent einten netten Erjatztuppentbeil (Эn= fanterie, (rtillerie, Irain).

Die Ferjonen, weldhe Das eigentlidje Seer bildoen, heifien Ferjonen des Soldatenftandes und jerfallen in 3 Şaupt= flafien: Dffiziere (Sienerale, Stabsoffiziere, 5auptleute und Rittmeifter, Subalternoffiziere), Unteroffiziere (mit unto ohne

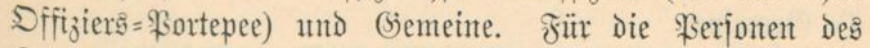
Soldatentandes gelten andere Beftimmungent, wie für 9) $i=$ litärbeamte.

\section{Bù Sunitätskorps.}

3ur Safredfthaltung, bejüglid, Sisiederherftelluntg Der

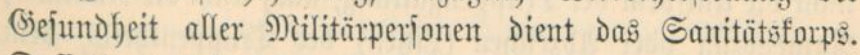
Sautitätßs= perionen

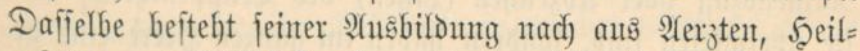
gefülffent unt Militïärfrantenmärtern, weldye alle Den (Eharafter Der Ferfonen des Soldatenftandoes tragert.

Die militärifiche (Eintheilunt ijt folgende:

Berionen beษ Soldater. ftanbes

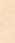
perionen

A. Santitätsoffizizere (Die Unteridjeioung vont Den Dffizierent fiefye aus Der Bergleidyung auf S. 12):

a) Dbere Militärürrite:

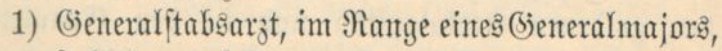
iteht dem gejammten Sceereš:Santitäts̄mejen vor;

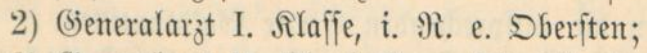

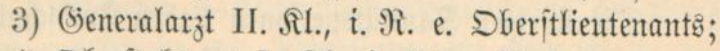

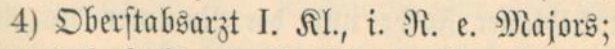

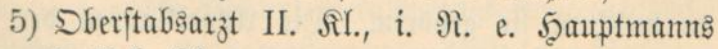
I. (3ieh).= II.;

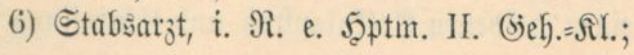


b) $\mathscr{A}$ fififtenzärazte:

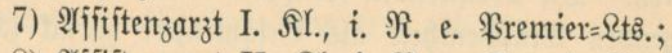

B. Unteroffiziere:

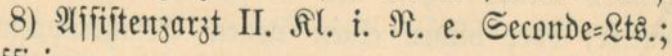

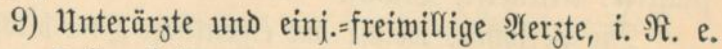
zeldowebels;

10) Sberlazarethgehülfe, i. R. e. Sergeanten;

C. Siemeine:

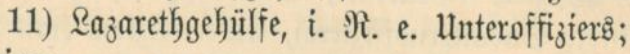

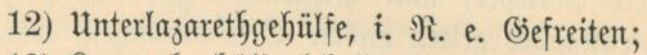

13) $\mathfrak{L a}_{3}$ aretbgehülfente hrlling ?

14) Militärfranfenmärter $\}$ i. $\Re$. o. (Semeintent.

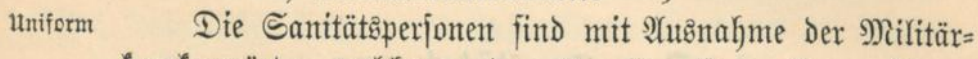

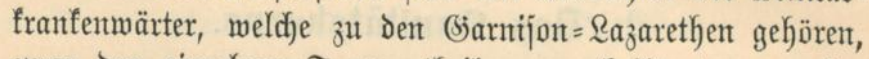
$z^{\mathrm{mar}}$ den einzelnen Iruppentheilen jugetheilt, tragen aber

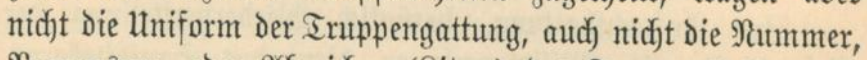

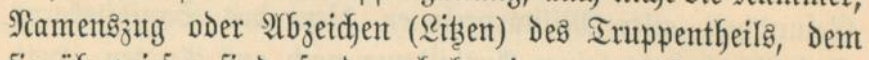
fie ïberwiejen fintb, fondern habent im ganjent Szeere eine ge= meinfame Utniform (Dunfelblauter Rod mit Dunfelblauem

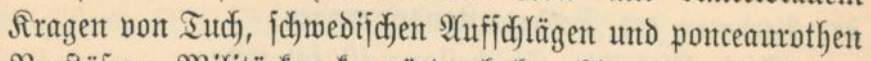

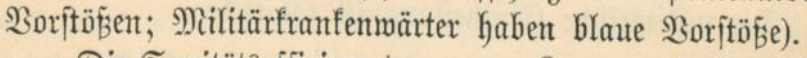

ber
Sanitätล̊. offizitere

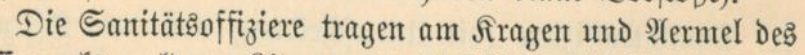
ISaffentrod's goldene Riţen und diejelben Sdjulterabzeidjent wie Die Dffiziere, mit Denent fie gleidjent Rang habent, Daz̆t Den goldenten Aešfulapitab und in Dent Epaulettes Glaue Sanmetfelder. Nat Stelle der Sillberfantillent Der Dffiziere tragen bie entipredjenden Fergte Bold fantillen.

ser übrigen Sanitäts. perionen

Die übrigent Eantiätäperjonen haben Iudjjdulterflappen

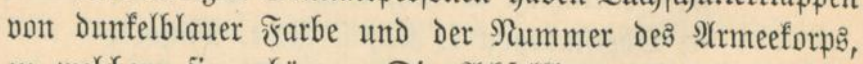

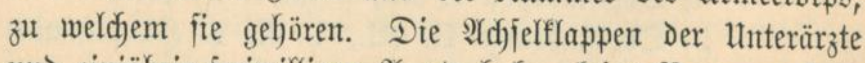
unt einjährig=freiwilligen $\mathfrak{A}$ er $x_{3}$ te $\mathfrak{h a b e n t}$ feine $\mathfrak{N u m m e r}$, font=

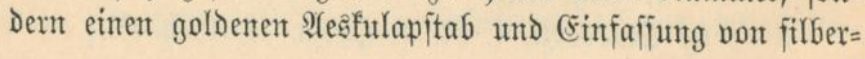




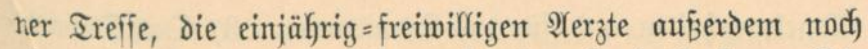
eite Einfafiung von Edjun in dent Randesfarben. Die Dbarlazaretb $=$, Razareth = und Unterlazarethgehülfen unter=

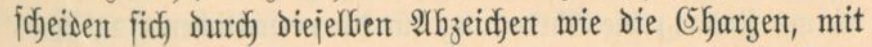
Denten fie gleidfen Rang haber.

\section{Air fjecresuermultutug.}

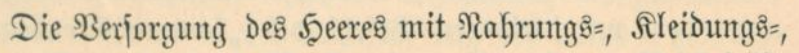

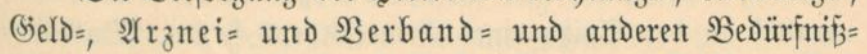

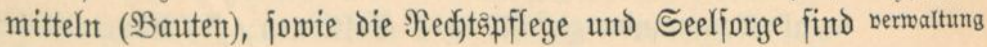
Fufgabe Der F̧ereşverwaltung, weldje vom Ririegsmint= fterium als oberfte Behörde bejorgt wird.

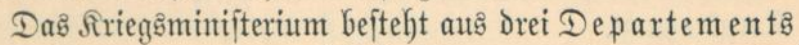

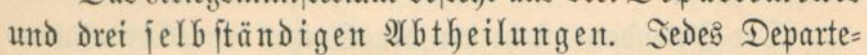
ment ift in (nid)t jelbitändoige) A(btheilungen eingetheilt, an Derent Spitze ein Shef fteht und in weldjent mehrere Dffizizere

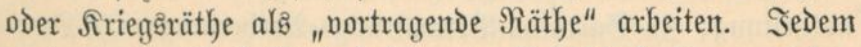

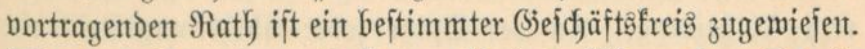

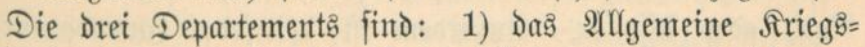

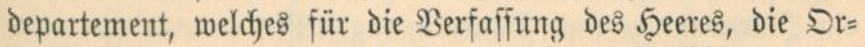

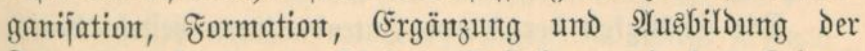

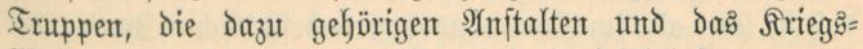

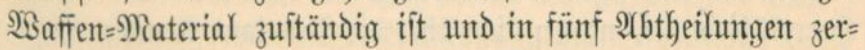

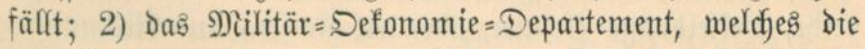

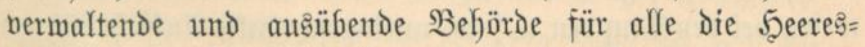
öfonomie angehenten Sachent und die Berpfleguttg des Seeres

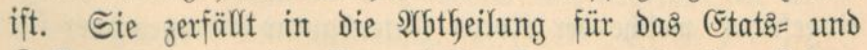

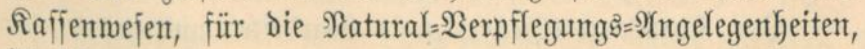

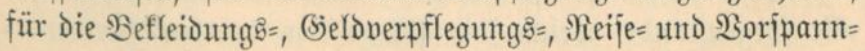

2ufgabe

ser

5eereas. minifiterium 


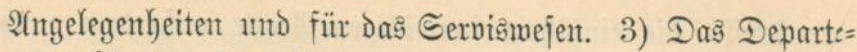
ment für dą Snvalidenmejent.

Die jelbjtändigen $\mathfrak{A b}$ theilungen dę friegsminite= rums finto 1) Die $\mathfrak{A}$ btheilung für Die perjönlichen $\mathfrak{A}$ (ngelzgen= Geiten mit Der (sebeimen Siriegstantzlei, 2) Die Afbtfeilung

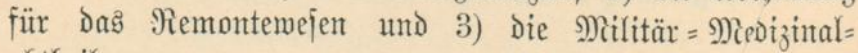
abtheilung.

Benteral.

Sommanoo

Die Frovinzialbehörden für die 5eerešvermaltung finto bie (seneral=fiommandos, weldje, wie jdjon pben gejagt, unter bem unmittelbaren Befehl des "fommandirentent (jese nerals" an Der Spitze eines 2 (rmee= fiorps ftelen. Das (5) $=$ neral = Sommando zerfällt in 4 Seftionten: 1) Sieneralftab; 2) भ(Djutantux; 3) Siorps= Yuditoriat; die 4. Seftion bildent Der Intentoant, Der forpsarzt mit fiorpsapothefer unt Der

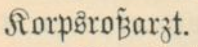

Sntentantur Der Intendant fteht an Der Epitze Der Rorpg̈=Sntent= Dantur, weldye in 5 Afbtheilungen zerfüllt: 1) Salfentabthei=

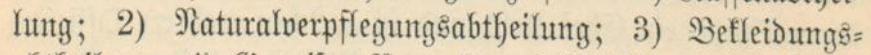

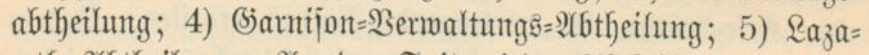
reth $=\mathfrak{A}$ (btheilung. $\mathfrak{A}$ (n der Spitze jeder $\mathfrak{a}$ (btheilumg jteht ein Rath oder Iffieffor. Aluberdem ijt bei jeder Intendantur ein Sntentantur = und Baurath angeitellt.

Die Ibätigfeit Der Rorps = Intendanturen bejteht Darin, dấ fie über alle im Sommandobezinfe befindlidjen Smeige der

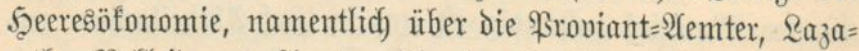

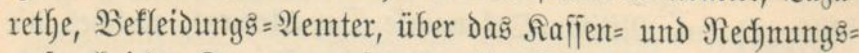

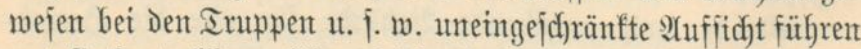
und fie fontrollirent. (Einte gleidye Behörde ijt bei Den Divifionent eingefübrt, weldje der Siorps= Sntendantur untergeoronet ift.

Siorpsiarst

unt

Rorpis: apotbefer
Der Rorpsarart (Gieneralarart) bearbeitet und trägt vor alle Giegentiände, weldye Die Pflege der franfen Sceeresper= fonen und die bei Dent Iruppent und Dent Garnijonent ange= 


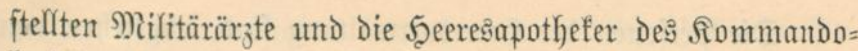

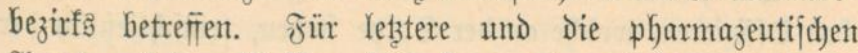

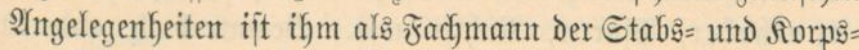
apotbeter zugemiejent.

Der STorpsarazt ift Dex 2orgejeb̨te Des gejammten in dent Sazarethen thätigen Santitäts̄perjonals unt Der Spothefer; er

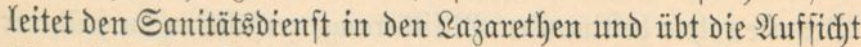

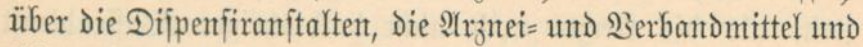

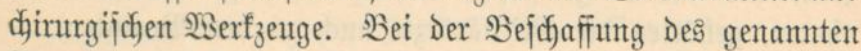
Materials wirft er in Der Ŝt mit, Daf die darüber von Det

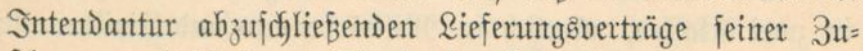
ftimmung bedürfen. EGenjo hat bei allen anderen Miá̧=

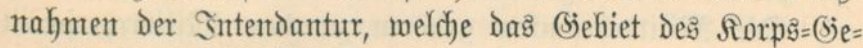
neralarztes mitberïbren, vor Der Enticheidung eine Bereint= baruty mit Dem forpsarat ftattzufindent.

Die Ffflid)ten deร Rorpsapothefers merdent unten näher befandelt.

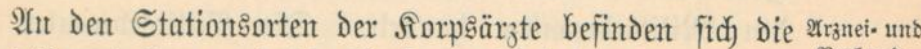

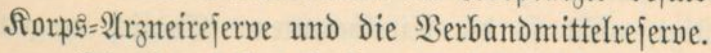

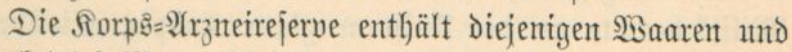
Berband = mittels. Rejerve Rebenbedürfnifje, weldye für den Bedarf eines ganzen $\mathfrak{\text { Irmee }}$ Rorps anf einmal bejd)afit und auf Beftellung an die ein=

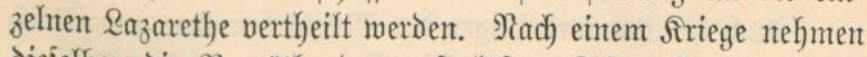

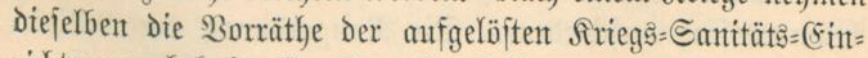

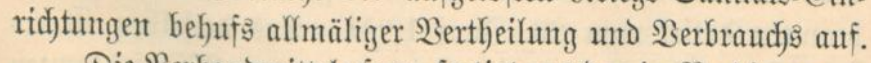

Die Berbanomittelrejerve fertigt aนร Den im Berdingungs:= wege bejdaffen Rohjtoffen Die Berbandomittel an und ver= theilt jie an Die einzelnen Iazarethe. Die Borjdyriften ent=

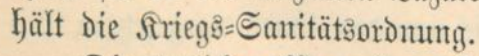

Die örtlidjen Berwaltungsbebörden fint unter antoren Die Irain= Depots, Die Siantijonbermaltung, Die Sarnijon=

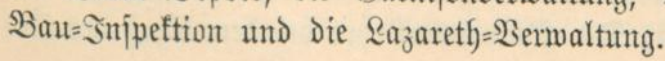
Ber = naltungs: bebörve 
Beamte

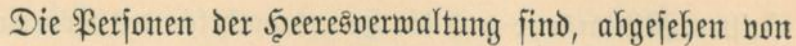

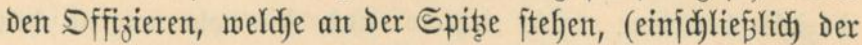

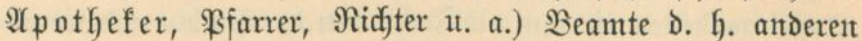
Beftimmungent untermorfen, al: die \$erjonen Des Soldatent=

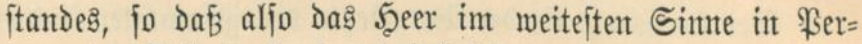
fonen Des Soldatenftandes (mit beftimmtem Militärrange) und Beamte (ohne bejtimmten Millitärrang) zerfällt. (In früberer

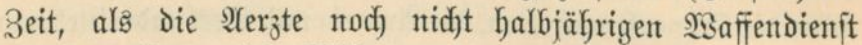
thatent, gehörten Die Mitlitürärzte aud zu Den Beamtent).

Unterídieb 3 wificient Dilitāas= ut:

(5ivil=

beamitelt ber seeres. verwaltung

Die Beamten Der \$̧eeres̉nermaltung zerfallen zutäd)jt in żmei אIlaffen, nämlid) in Mitlitärbeamte und (Sivilbeamte der Sceerešverwaltung.

Die Militärbeamtent gehören zut dent Militärperjonten und Gaben Den Militärgeridjtşitand in Strafjadjen (Militär=

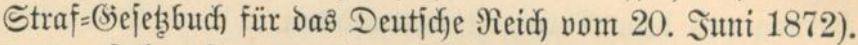
Fenter ftehen fie unter Der Disziplinaritrafordmutg für ba

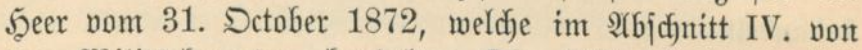
ঠen Mitlitärbeamtent hantolt. Die Millitärbeamten fint jerviäberedytigh, nidyt fo die Sivilbeamtent. (Endlid) tragen Militärbeamte an der Uniform glatte Sinöpfe, Sivilbeamte aber, ment ifnen eite Uniform vorgeldyrieben ijt, Ströpfe mit Dem $\mathfrak{2}$ Sappenabler.

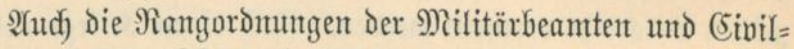
beamten find verjdjiedente. Die Mangorbmung der Mitlitär=

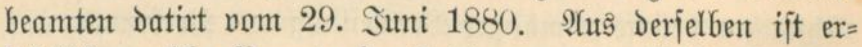
fidftlid), meldje Beamte im Frriedent zu Den Militärbeamten

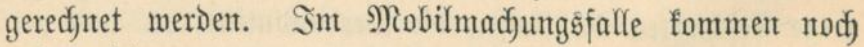
andere binzu.

I. Militärbeamte, meldje nur Den ifynen vorgejeşten Mi litärbefehlähabern untergeoronet fint:

A. Shere Nitlitärbeamte (mit Dffizierêrantg):

1) Der Bureaunorjteher bei Dem Çhef des (Bie= 


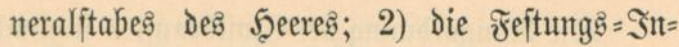

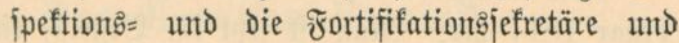
die bezüglidfen âjifitenten; 3) die 3ahlmeifter; 4) Die fiorpse und Dherrofärate; 5) die Dber= apothefer; 6) die Stallmeijter.

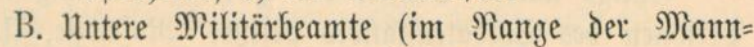
id)aften vom Felomebel abmärtz) :

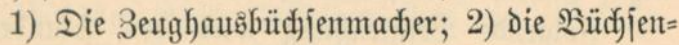
madjer unt Sattler bei Dent Iruppen.

II. Militärbeamte, weldabe in einem doppelten Interord=

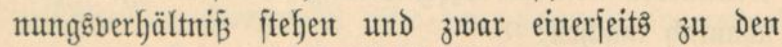
ihnen vorgejetsten Mitlitärbefehlähabern, andererjeitร zut ben ihnen vorgejełsten höheren $\mathfrak{B}$ eamten oder Behörden:

A. Dhere Silitürbeamte (mit Dffizierşrantg):

1) Die Rorpsintentoanten und Die Borftände Der Divifitonsintentanturen und Deren Bertreter;

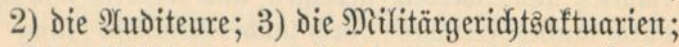

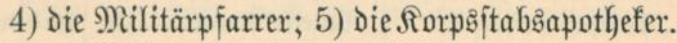

B. Untere Mitlitärbeamte (im Range Der Mant=

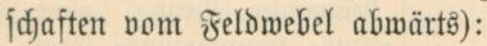

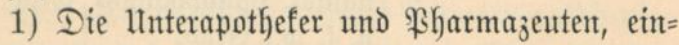
id)lieblidy Der einjährig=fremilligen; 2) Die $\mathfrak{M}_{i=}$ litürfüijter.

III. Mitlitärbeamte, meldje mur den ihnen vorgejeb̧ten höhe= ren Beamten oder Behörden untergeorbnet fint:

1) Der Bienteralauditeur des 5ุeeres und die Räthe des (Sieneralauditoriats; 2) die Sntent=

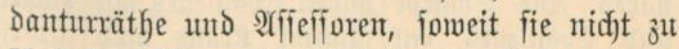

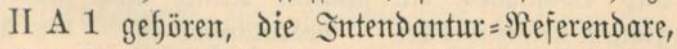
Intendantur = Sefretäre, Intendanturr $=$ Regi tra $=$ toren und Die bejüglidjen a(jifitenten; 3) Der

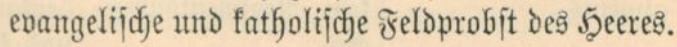




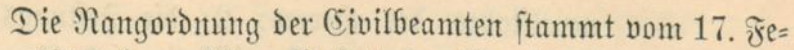
brutar 1817 her. Niant ift jeţt im Begrrifi, eine nette aus= zutarbeiten. Die Ciwilbeamten tretent, went fie im Siriegğ=

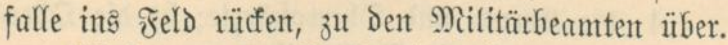

Einige untere Stufen der Şeerešverwaltung find mit

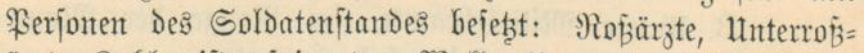

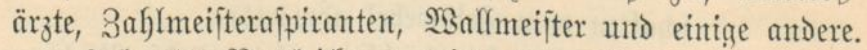

unteriđjieb zwifichen

Difizizieren,

Sautitătకి.

offizizierent

unb Militär.

beamten mit (Truppent $=$ ) Difiziere Diriziers: rang

werden von einem

( Truppent $=$ ) Dffiziter $=$ Rorp $\left.*^{*}\right)$ gemählt. Sie unteritehen Efrenge= ricften uns merbent Durch Iandesherrlidjes "Patent" exnannt.

*) Ein (Iruppen=) Dffit zierforp̧ wir๖ aus fămmtliden (Iruwpens) Difizieren eintes Regiments ธeวw. einteต Referve $\cdot$ Ranswehr $=$ Batail= Ions ober äbulidser (Ein. beiten gebilbet.

( Iruppent $=$ ) Dffï 3 iere

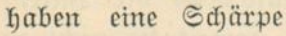
uns Epaulettes mit glattem Rranz und Der

Nummer bezw. Dem Ramensaztge ifres $\Re e=$ gintents.
Sanitätsoijijiere

merben von einem $ఠ a=$

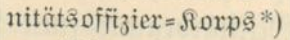
gewö̈blt. Sie itnter= iteben nicht Efren= geridften unto werben Durdy Ianbes ferrliches "Patent" ernannt. ЭGr Difiziersang hat einen bejtimmten (Eieute= nants $=$, auptmann $9=$ 1t. 1. w.) Girad.

*) (Sin Sanitätsofinisier= forps befteht auts iäumt. lid)en Ganitätsoffïjieren einter Divifion.

\section{Sanitüts̊ofifiziere}

Gaben Epaulettes mit glattem firanz und 2lesfulapitab in jammtblauen frelde, aber feine Schärpe unऽ fein Regimentsabzei= d)en.
Difiziziersbeamte werDen nicht gewäblt und unterîtefen nicj) Ehrengeriáten. Sie werden Dutrd Priegs minititerielle "Beital= lung" ernannt uns haben mux allgemei= nen Dffiziersrang ofne bejtimmten Srad.

Difiziersbeamte haben Epaulettes mit geprebitem $\Re_{\text {ran }}$ und Dem Alslexichild in je nac) Dem 3weig Der Bermaltung verifjie $=$ Denfarbigem Felde

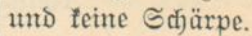




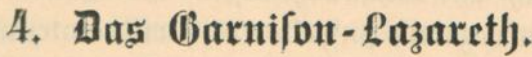

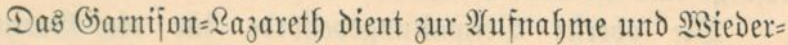

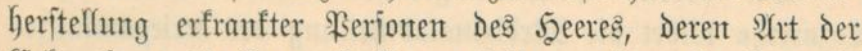

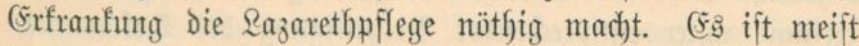
3wect

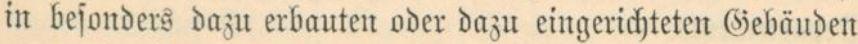
untergebrad)t. SYlt Der Spibe defielben fteht ein Shefarzt.

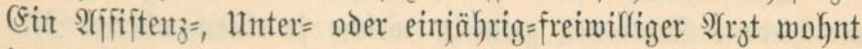
in Demijelben als wad)thabentoer $\mathfrak{A}\left(z_{3} t\right.$.

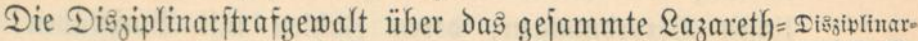
perjontal und die Siontrolle iiber Das $\mathfrak{R}_{3}$ areth ift Dem fiom= mantoantent, bejw. in offenten StäDten Dem Bananijontältejtent eingetüımt.

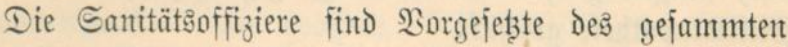
Ferjontals. Sisiro ein Unterarat oder einjübrig=freimilliger

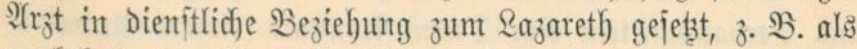

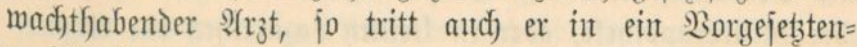
verbältnif́.

Der Dienit im (jarnifont=Razareth) zerfällt in zwei ver= idjiedene 3meige: Sanitätşoienjt uno Berwaltunggsoienft. Santiätb̊=

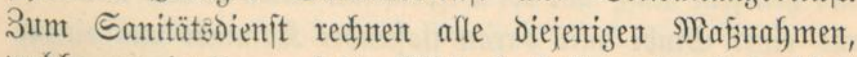
weldye unmittelbar auf bie $2 B$ iederheritellung Der Bsejundheit Der Fffleglinge und Errhaltung Der Bjejundheit Der Fffleger

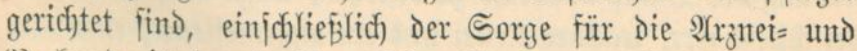
Berbanomittel unto djinurgifden $\mathfrak{B S e r f}_{3}$ entge. (Ex wiro bejorgt von Dem Santiätsperjonal unto dem Militärapothefer und ijt Dem Rorpsan

Der Sermaltungedienft (Die Siaffen= unt Defonomie=

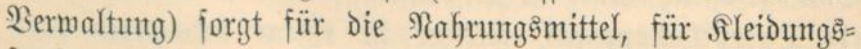
ftürfe, Bremmaterialien, Utnterhaltuntg Der Bjebünde und Deڤ verbältniffe sienit (orumbeigentfums wie Des $\mathfrak{L a j}_{j}$ arethgeräthes, und Das gejammte

Ber: waltungs sienit 


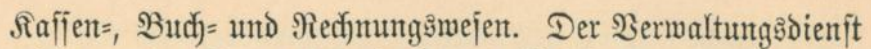
wird von Den $\& a_{z}$ areth)= Snjpeftoren (Civillbeamte) und anderen bejorgt und ijt Dem Rorpsintendanten unteritellt.

Die oberfte Bermaltungşbebürde für die Sarnijon=

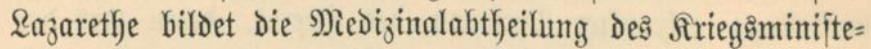
fteriums unter entiprechender Betheiliguntg Deş Defonomie= Departements.

Sranfen. behanblung

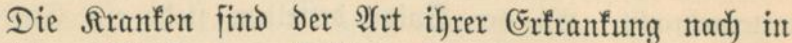
werjodiedente Stationen getheilt, weldye je einem oberen ) $i=$ litärarjt als veroronendem $\mathfrak{A}$ rzate unterjtellt fint. Die arznei= lidjen Beroromungen, Deren Bablen mit Budfjitaben ausి= gejd)rieben werden müfîen, werden von einem unteren Militürarate in ein für jede Station bejonderes Beroronungä= bud eingetragen, vom Dberarzt gegengezeidynet und zur Millitärapothete gejodjift.

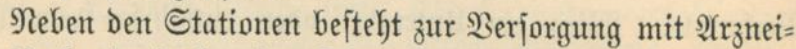
und $\mathfrak{B}$ erbanomitteln eine Dispenfiranftalt $(\mathfrak{N}$ ranei $=\mathfrak{N}$ (njtalt?) oder Militürapothefe, oder in fleinen $\mathfrak{L}$ azarethent ein $\mathfrak{A}$ rznei= und Bandagen=Sabrant.

Einridstumg Der Difpenīir. anitalten

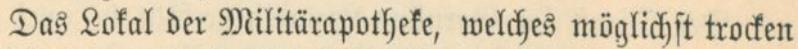
und hell jein muí, hat bei Dijpenfiranjtalten 1. und 2. SIlafje aนtร einer Stube mit daran ftofienter Rammer und einem, womöglidy hiermit in Berbindung ftehentent Raume zur $\mathfrak{A} u f=$ ftellung des Dampfapparats zu beftehen, wozu nod) ein fleiner

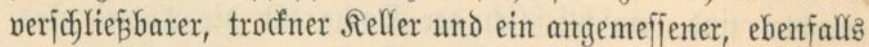
verjad)liefibarer Bodenraum fommt.

Bur Dijpenfiranitalt gehört ein Repoittorium nebjt

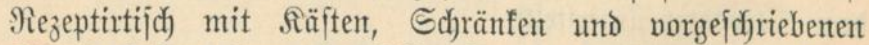
Standgefäjen; ferner in Dijpenfitranjtaltent 1. und 2. Slajie ein Dampffodjapparat, weldjer in einem bejonderent Dazıt

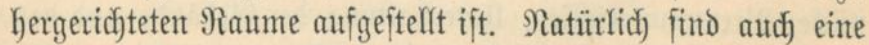

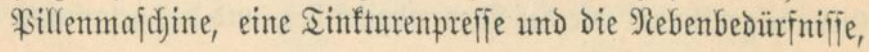


mie Şläjer, Rorfe, B̉indfaden, Sdyreibmaterialien, fowie Iijade, Stiible u. j. w. vorbanden. Ein in jeder Dijpenfir=

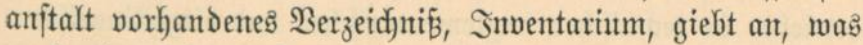
vorbanten jein muí.

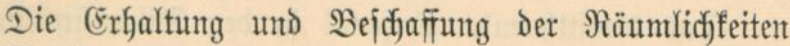

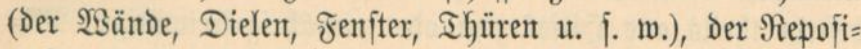
torien, Iijade, Stüble, Standgefäpe und anderer (Sierätbe und Der Sdyreibmaterialien gehört zum Berwaltungsbienjt und ijt Der Sorpsintendantur bezm. Den Injpeftoren unterjtellt.

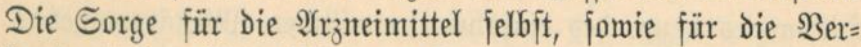
bantomittel, djirurgijdyen Snitrumente, Deren Sinnabme,

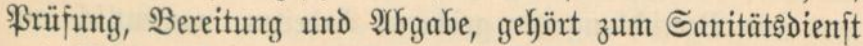
und ift Dem Rorpiggeneralargt unterftellt.

Die Dipenfiranitalt ift Bormittage von $9-12$ uhr und

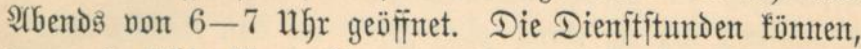
ment eڤs nüthig ift, verlängert merden. $\mathfrak{A}$ uber Der feitgejetsten

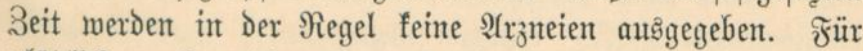

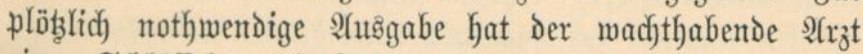

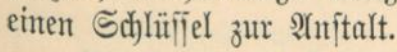

Die Empfänger der $\mathfrak{A}$ żneien find theils die im $\mathfrak{R} \mathfrak{a}_{z}$ areth liegenden, theils die bei den Iruppentheilen Gleibenden jo= গrąneiEmpfăınger

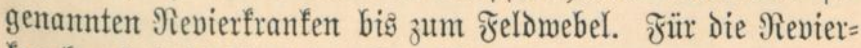
franfen befteht für jedes $\mathfrak{B}$ ataillon ein $\mathfrak{B}$ erordmungsbud

Die Dffiziere und Beamten, jomie die Frauten und

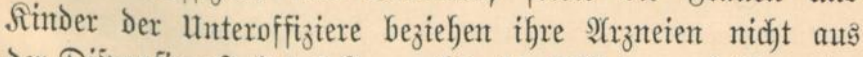
Der Dijpenfiranjtalt De: $2 a_{z} a r e t b \xi$; Die Dffiziere und Beamten mur Dann, went fie fid in Sazarethpflege befindent.

Die Reitung Der Dijpenfiranitalt mirb, went Der Shef= arzt nidjt etwa jelbjt die Reitung übernebmen will, wenn möglich, einem bei ber Stationsbebandlung nidyt betheiligten

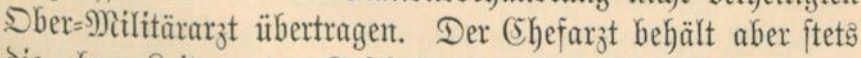
Die obere Reitung Der (Siejd)äfte Der Disipenfiranjtalt.

Dientit in ber Difpenfix: anftalt 
Die Dbliegentheiten des ärgtliçfen Borftandę der Dijpenfitr= anjtalt umfaffen die Beauffidtigung deş gejammten Dienft=

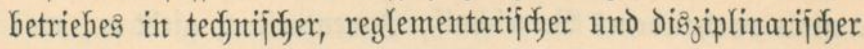
Şinfidut.

Das dienjtthuende Ferjonal in den Dijpenfiranftalten

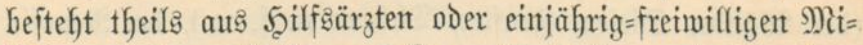

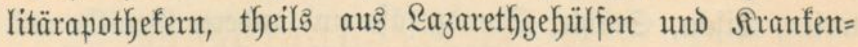
märternt.

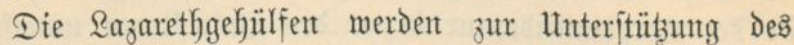

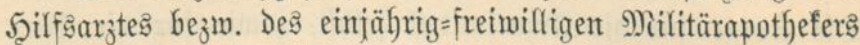

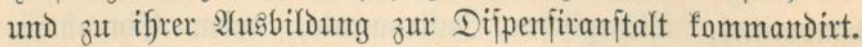
Bur Berrichtung Dex gröberent $\mathfrak{Y}$ rbeiten, namentliç zur

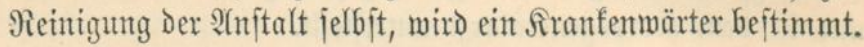




\section{Die Soeresapotheker.}

Die Şeereşapothefer müffen ifrer Dienfttplidut als ein= jäbrig = freimillige Millitärapothefer genügt haben. Mit Der ISaffe gebiente approbirte $\mathfrak{A}$ (pothefer Dürfen im Frieden nidjt forde gebient

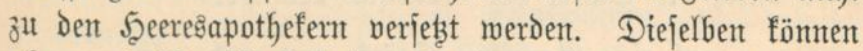
baben

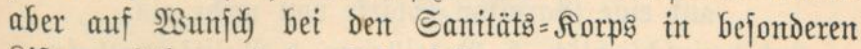

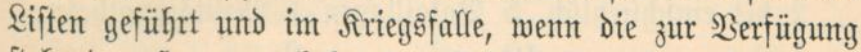

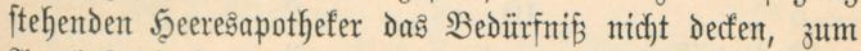
Ipotheferdienft herangezogen merden. (Dieje Ueherweijung

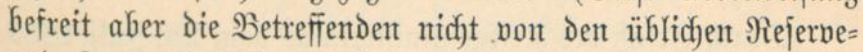
und Randwehr=Utebungent.)

Die Raufbahn der Şeereşapothefer umfá̧t 5 Stufen, \&aufbahn von Denent bie 2. und 3 . im Frieden mur im Beurlaubtent= ftande befteyt:

ber

Seeres. apotbefer

1) (Einjährig=freimilliger) Militürapothefer;

2) Ututerapothefer;

3) Sherapothefer;

4) Stabsapothefer;

5) Dberitabs̊apothefer.

Die unter 1-4 Gientantenten gehörent zut Dent Militär= beamten, nur Der Sberftabsanotbefer ift Sivilbeamter.

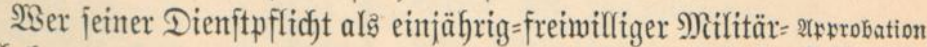

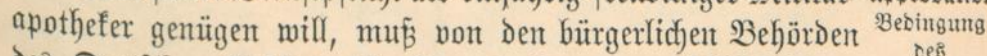

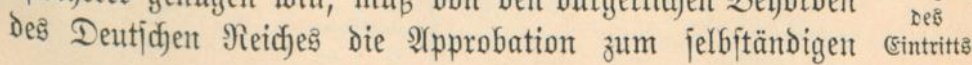


Betriebe einter Apothefe erhalten habent. Siant diejelbe nidat bis zum 1. Dftober Desjentigen Sabres, in weldhem der Dienit=

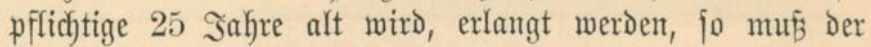
Dienftpflidjtige beim Siriegsminifterium durd) die Errjatz=

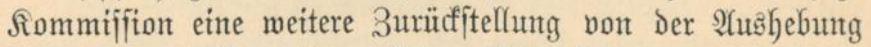
3u erlangen jutden oder mit der 2 saffe bienten.

Eintritt售it

Der Eirtritt erfolgt int Der Riegel am 1. Itpril oder am 1. Dftober. Dody fintent bei vorbandenen $\mathfrak{B a f a n z e n t}$ Einftellungen zu jeder 3eit ftatt.

Die Sorte, bezm. Die Siantijon=Razarethe, an weldjen Militärapothefer eingeftellt merden, fowie bie 3ahl Derjelben wiro Durd) Die Stärfe Der Gäarnijon bedingt:

auf eine Ģarnijont = Stärfe vont mefrr als

2 bis einjdylię̧lide 5 Bataillonen 1 Militärapothefer,

5

10 Bataillonen

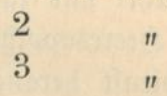

Eintrittio orte

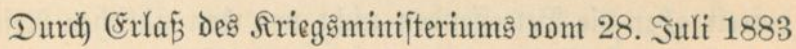
ift bie 3ahl Der Millitärapotheferftellen vermintoert und be=

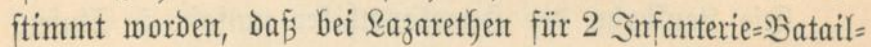
Ione mit Stab oder eine an Siopfitärfe diejen entipredjende (S)antifion bis auf 23 eiteres Millitärapothefer niddyt mehr ein= geftellt werden. Dent Razarethen, mit weldyen eine hygientijd)=

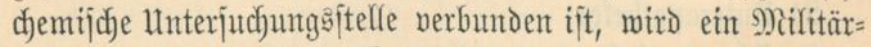

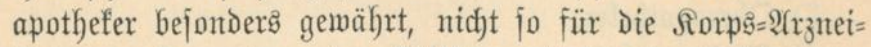
Rejerve. Danad) merden Militürapothefer eingeftellt: 


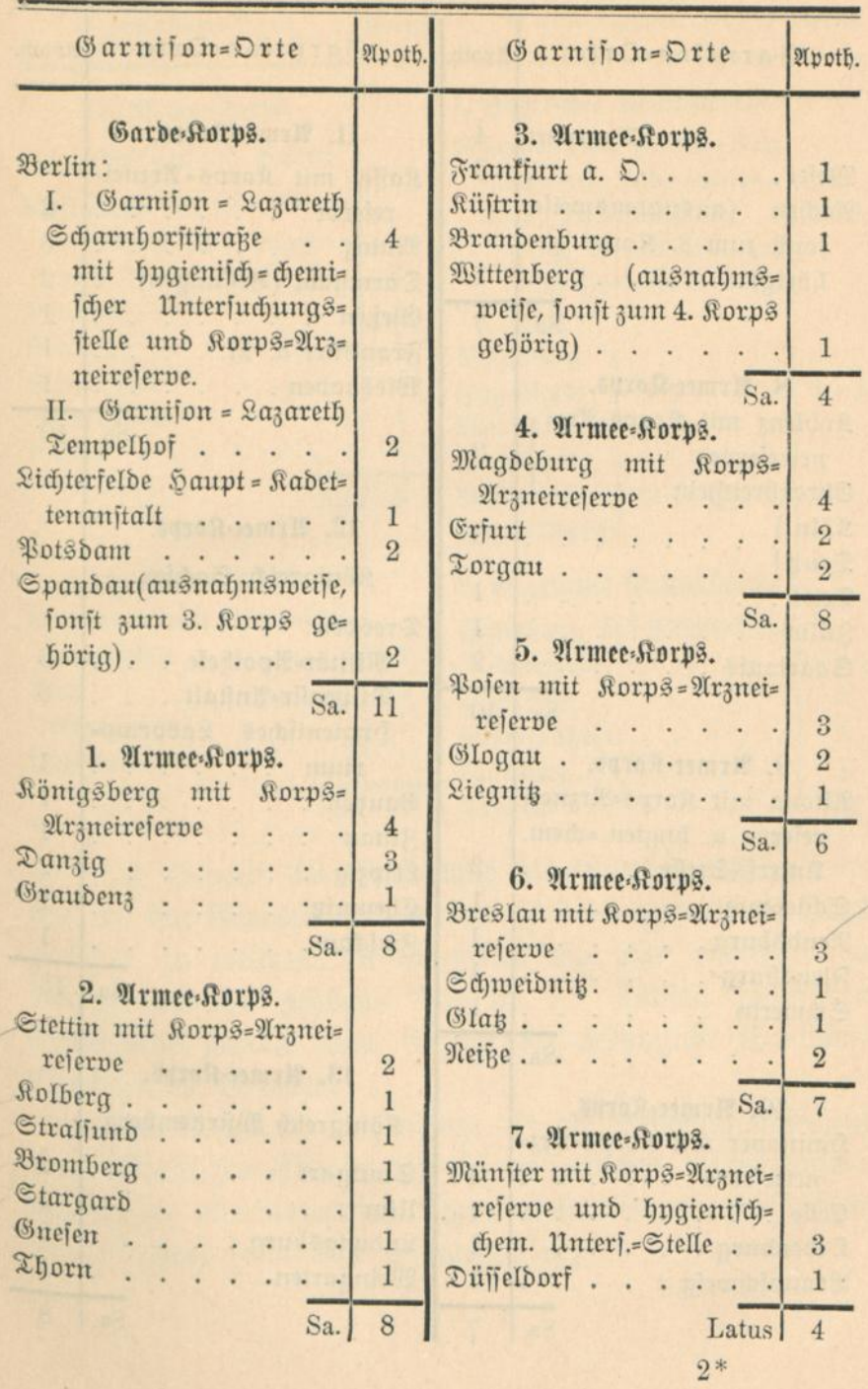




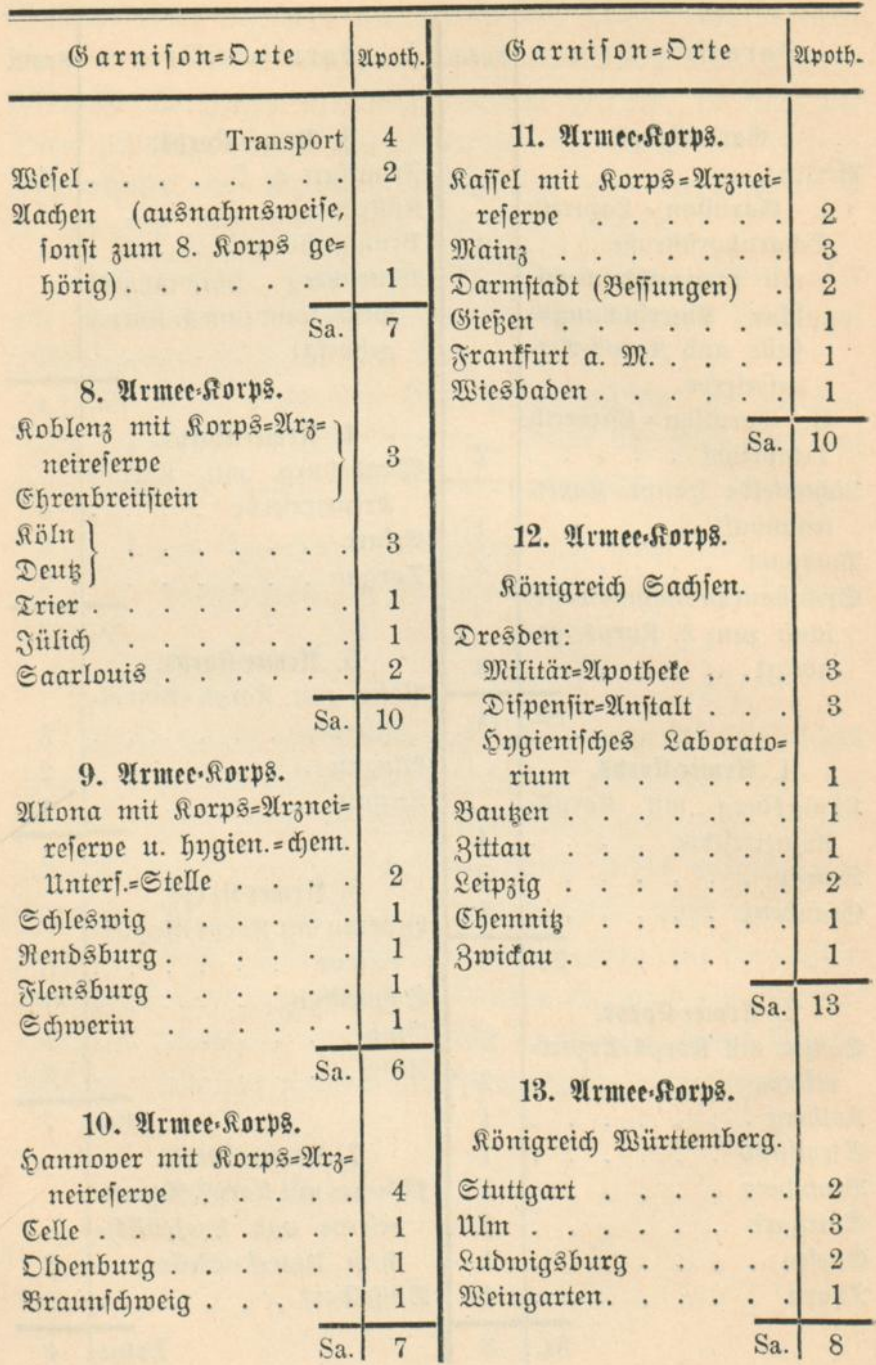




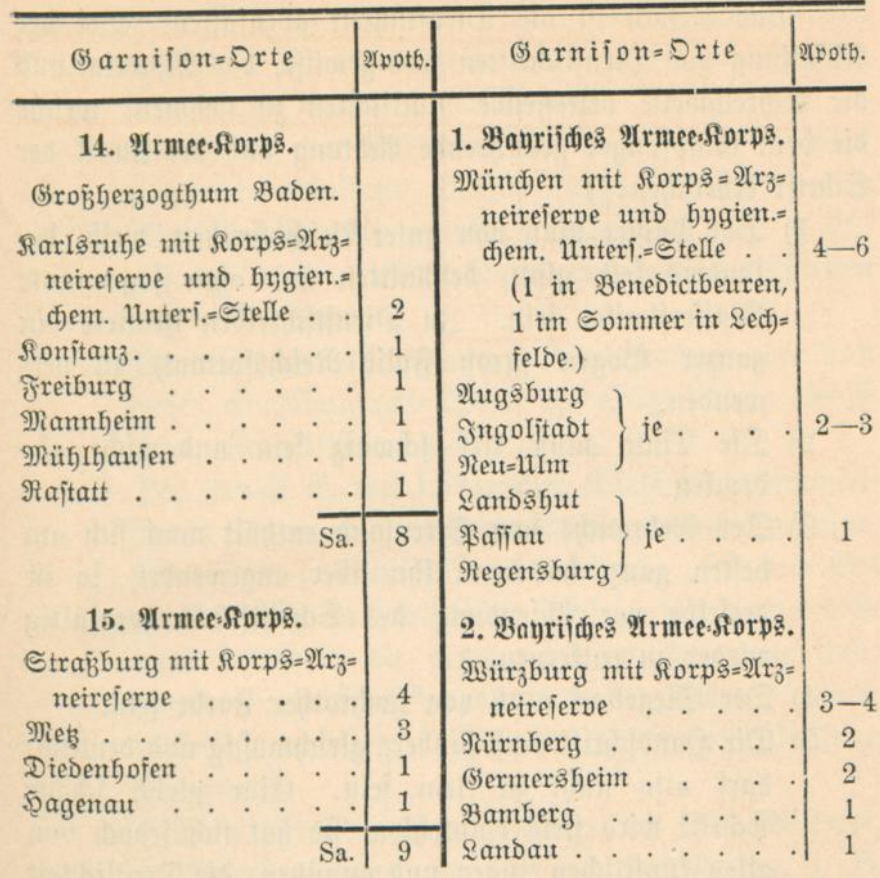

Die (s)ejuche um Ëinftellung als 9illitürapothefer wer= Dent an Den (S) geridjtet, ju weldjem die Bianijon, wo mant eingeitellt ju

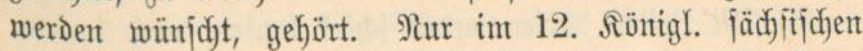
शrmeeforps wendet man fid an die Rïniglidye Suntiätš= Direftion in Dreşden.

Dem (Sejud ift beijufügen: 1) Der Beredytigungşidjein 孔um einjäbrigen Dienit, 2) ein polizeilidjes Sittenjentuní (Eintritte8. Beuguniffe und 3) Die pharmazentijude $2(p p r o b a t i o n$ oder ein vorläufiges Beugnifi über bejtandente Etantäpriffung. 
Regelnt für Dent id)rittlidien

Berfebr mit borgejestent Behörben

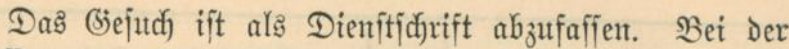

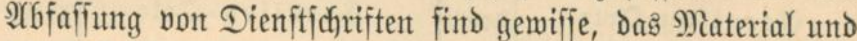

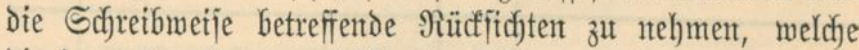

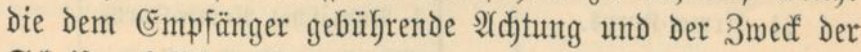
Sdjrift erbeijdjen: *)

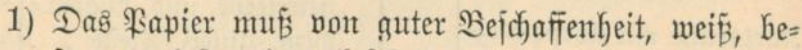
jonders feit, glatt, bejdnitten uno ofute jogenannte SBafjerftreifen jein. 3u Dienfifjureiben ift ftets ein

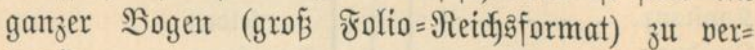
mendent.

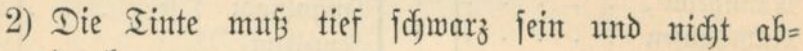
Drutfert.

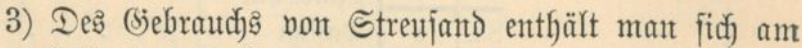
bejten ganz, hat man ifn aber angemendet, jo iit

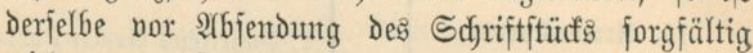
mieder zut entfernent.

4) Der Siegellauf muf́ von hodjrotber Furbe jeint.

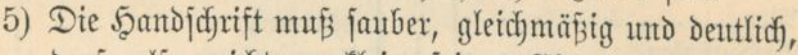
Darf aljo nidjt zu flein jein. Eine gleid) jodjüte Sdyrift mird ftets empfeblen, fie hat fid jedody nont allent fünftlidyen Bügen und untützen, die Deutlidffeit

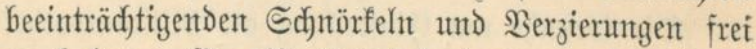

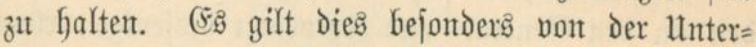
jodyrift.

6) Die Beilent Dinfent meder jujief laufen, nod) jo eng ftehen, daf́ die Shudfitaben in die der anderen Beile Gineingejdyrieben fint. Die $\mathfrak{a b j t a ̈ n d e ~ d e r ~ B e i l e n t ~}$

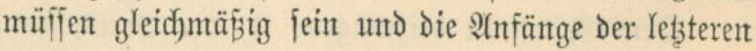

*) Entnommen Der: Jnitruftion für Die Dffiziere unઠ Santäts=

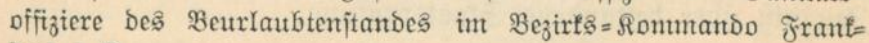
jurt a. D. 
jenfredyt unter einander itehent. Meber Den oberjtent und unter der letsten Beile einer jeden Seite eint etwa Drei Finger breiter, freier Raum.

7) Die Ramen von \$erjonten, Drtidjaften und Mionaten

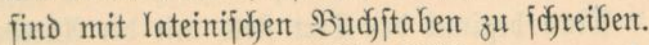

8) Siemifile, allgemein übliche stbfürzungen, wie z. B.,

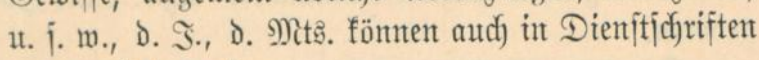
angementbet werdent.

9) Berbefferungen, Rabirungen, Durdfjtridjente oder ftatt Defien eingeflammerte $\mathfrak{W o r t e}$ und Säbze find in bienit= liç)en Edyriftitü(fen nidj)t zuläjfig.

10) Der Infalt ift mit joldatijdjer Sïrze in beftimmtent furzen Säben, ohne unü̈ze Erörterungen abjufafien. Şorte wie "mittheilen" , "bemerfen", "mit Dem $\mathfrak{B} e=$ merfen, find im jufriftlidjen $\mathfrak{B} e r f e h r$ mit Borgejesten nidjt ftatthaft; Die gebräud lidjiten Beitmörter fint: "melden", berichten", "portragen", "bitten", "über= reidjen", "porlegen". Fremowörter find möglidjit zut nermeident.

Ein Dientifidreiben fann entweder an eine Beförde,

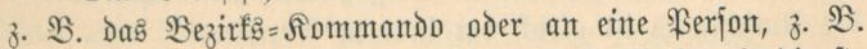
Den $\mathfrak{B} e$ zirfê=Sommandeur geridjtet jeint. Briefe in rein dienjt= Yidfem Intereffe find an bie Befjörde, Der Briefmedjel in eigenten $\mathfrak{A}$ (ngelegentheiten, namentlich) (siejudje, fomie Mitthei= lungent vertraulicjen Intbalts find ftets an die \$erjon zut ridytent.

3u Dientidy)reiben ijt Der Bogen von oben nady unten gentau in Der Mitte zu brechen und Der Sert mur auf die redyte Seite vom Butud) zu jujreiben. Drt und Datum mer= Dent in alfen Dientbriefen oben redjts in eine Beile, zeilen=

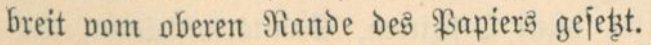

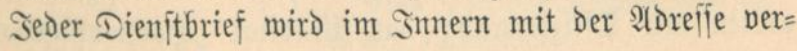


jefen und zmar iteht diejelbe redjts

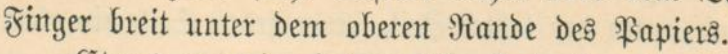

Etmas unter der imeren भideffe beginnt ber Iert. Der= jelbe darf, went ex nur furz ift, nidjt zu hod) angefangent

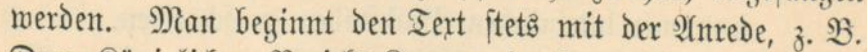

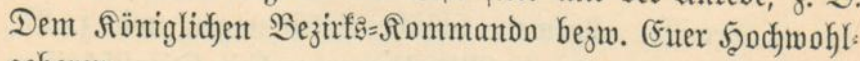
geboren.

Die Unterjdyrift hat in eriter Beile den Namen und in zmeiter und, wenn nöthig, britter Beile die 5harge ju ent= Galtent.

Anlagen, ๖. h. Dem Briefe beigefügte andere Sdyrift= ftüffe, müfien im Iert ermähnt merdent. Da, wo im Sdyrei=

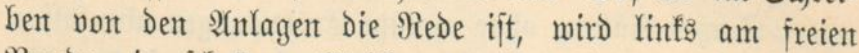
Rande ein jadyräger Stridj) gemadyt, über meldyent, ment mebr als eine Antage vorbanden, die 3ahl derjelben an= gegeben wird.

Sedes Dienftjodreibent ijt zur צbjendung aujer der Ränge nadj) einmal in der Mitte ju brechen, mit S(usntahme pout

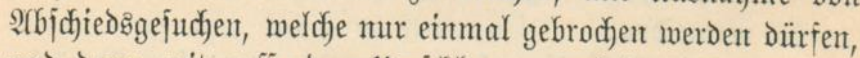
und dann mit paffendem Umjalag zu verjeben. Die Ardreffe

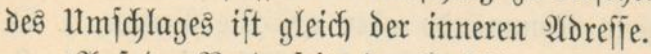

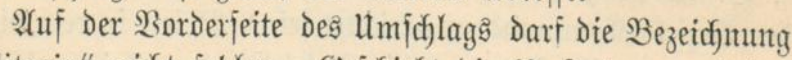
"Militaria" nidjt fehlent. (Sjejdjeht die Berjendung mit Der Poit portofrei, fo ift der Brief zu verfiegeln und auf ber Pürfjeite der Bermerf hinzuzufügent:

"In Ermangelung eines Dientifiegel:

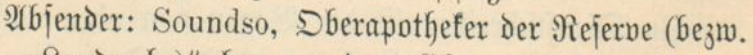
Sandmefr)" bezw. andere (5harge.

Meldungen auf Foftlarten find nidjt ftatthaft. Stadt= briefe mülfen freigemadyt merdent.

Eฐ ît Sitte, allen Dffizieren, Sanitätsoffizizerent, $B e=$

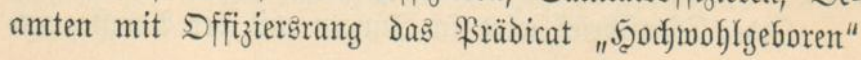


zut geben; eigentlidi gebührt daffelbe erift Den Graden vom Stabs̊offizier aufmürts.

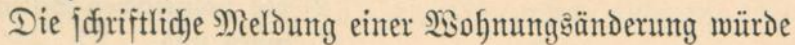
demradi) 3. B. lautert:

Drt, Den ...ten Monat $18 \ldots$ 2(n

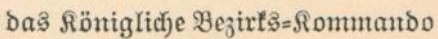
out

Dem Röniglidjen $\mathfrak{B e}_{3}$ irf: $=\Re$ om $=$ mando melde idf gan geforiamit, Dá ich meinen $\mathfrak{B o h n i t s ~ a m ~ . ~ . t e n ~}$ ๖. Mts. von ...... nad) .. (Drt).

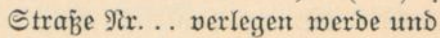

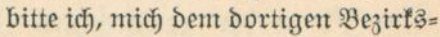

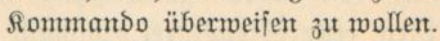

Soundso,

Dberapothefer Der Rejerve

(2antomefr).

Fortofrei finto mur Dann Dientfbriefe, wemt beide, $\mathfrak{A} b=$

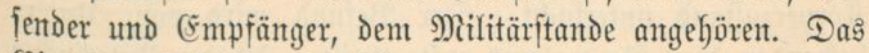
(Eintrittsgejud ift aljo nidyt portofret.

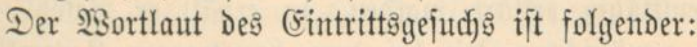

Drt, Den .. tent Monat 18 . .... Straje $\Re \mathfrak{x} . .$. $\mathfrak{A} \mathfrak{n}$

Den Röniglidjen Genteral Rorpsarart Des .. ten 2 (rmee $=\{$ orps, Ritter Serrn Dr. ......

\section{5ochwohlgeboren.}

(Ener 5ुo(t)woblgeboren bitte id) unter Beifügung
ISortlaut einer

Meloung einer झुohnfiti. ănberung
Eintrittgి. gejuds 


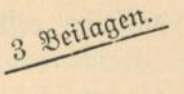

1) Des Bereditigungid foines zum einjäfrigen Dienjt,

2) Des polizeilidjen Sittenzentg= nijies uns

3) Dex pharmazeutifichen $\mathfrak{2}$ (ppro= bation (oder bezügliduent $\mathfrak{B e}=$ icjeinigung)

geforiamit, mix bie Albleijung meiner \$ecresdienjtpflid)t als ein= jährig=freimilliger $\mathfrak{M}$ ilitärapothefer im Garnijonlazareth ş .... vom 1. Alpril (Dftober) 18 . ab ge= jitten 3 ut wollent.

Rame

Ipothefer.

Sërwerlidie Muiterumg
Sit zut Der gemünjodten Beit und an Dem gemün fdjtent Drte eine Stelle frei, wontad) man fid worher entmeder bei Dem bejïglidjent Siorpsitabąapothefer oder bei Der Berwaltung ๖еริ $\mathfrak{L a z}_{3}$ areths erfundigen faum, fo wiro der Dienftpflidtige

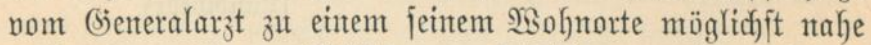
mofntentoent oberent Militürarzt befohlent, weldyer jeintent Sïrperzuftand unterfuddt. Der Befunt wird vom Unter= judjentoent dem Gieneralarzt mitgetheill. Sit Der Unterjudjte für dienjtfähig erflärt wordent, fo erfält er Befehl, fíd

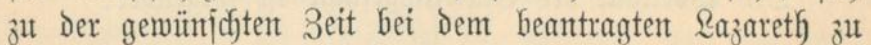
meldent. Sit er für untbraudj)

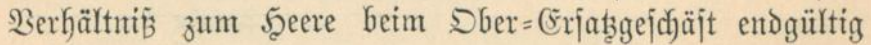
entidjiedent.

Der Eintrittsppflidjtige mufí in Uniform antretent unt, ba er als Mitlitärbeamter z̆ Dent Militärperjonten zählt, bie militärifiden Sूomteurs und $\mathfrak{A n j}$ tantogregeln befolgen; ebenio Die übrigen Şeeresapothefer iffer (Sharge entipredjend. 


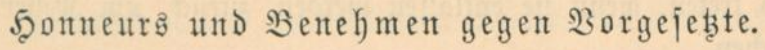

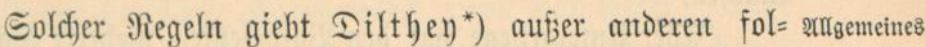
gente: Die Şontents find bie vorgejdriebent äufieren Ehrenbezengungen des Untergebenen gegen Den $\mathfrak{B}$ orgejeţten,

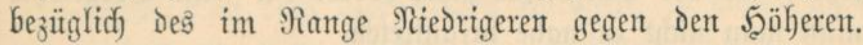
Bu bemerfent ift, daj Den entipred)endent (5 hargen Der Marine Die Sonnents von Eeiten Der Ferjonen Des Ranbheeres ebenjo out ermeijen fint, wie Den 9 (ngehörigen des Seeres und um= gefebrt.

Seder Zorgejetzte oder 5ä̈hergeitellte ift verpflichtet, die

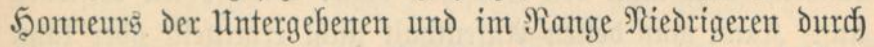

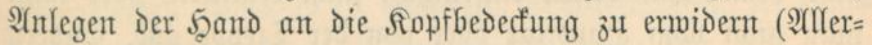
Gödjite Rabinetgordre vom 15. Jan. 1817).

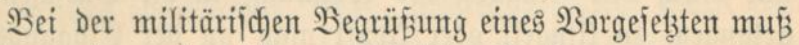
Der Untergebene ein völlig militärijdyes $\mathfrak{A}$ eujere zeigen. Un=

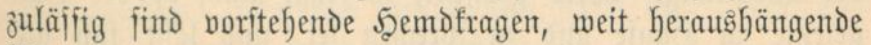

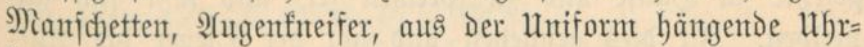

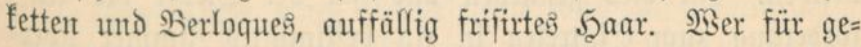

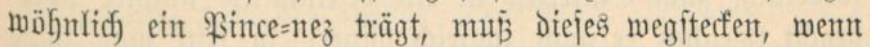
er ou grüfent hat.

Dffiziere legen Die 5̧and an bie Siopfbedecfunt, ment

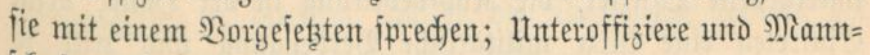
idjaften thun dies nicht.

Dffiziere fönten fidf) mährend deร (simbes, meldjen fie ftebend abgeben, währent 2orgejetzte vorübergehen, ober wenn fie von Dem 30 rgejeşten nad) beendigtem (siejpräd) entlafien merden, verbeugen; Interoffiziere und (siemeine thut Dies nidgt, jondern nefment nux eine ftramme Şaltung an

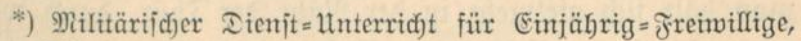
Rejerve $=$ Difizier:2lipiranten und Dffizitere Des Beurlaubtenjtandes Der Deutichen Infanterie. 
und treten, wenn jie entlafien merden, zurïd, um fiefrnt zu madjen.

Seder Untergebene hat Den Borgejeşten mehrere Sdjritte

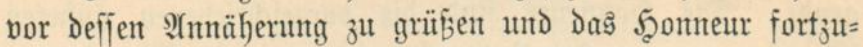

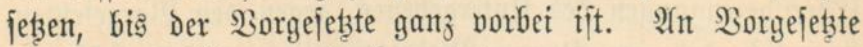
muf man nidat zu nabe berantreten.

Dffiziere madjen beim $\mathfrak{B e g t r e t e n t ~ v o n ~ e i n e m ~} 2 o r g e j e t z t e n t$

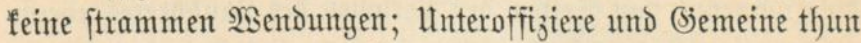

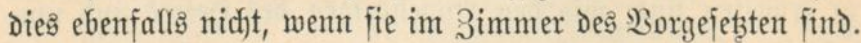

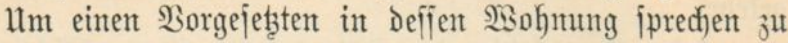
fömen, jud mant Den Burjden bezw. Dienftbotent Deflelbent auf. Siant mant Defien $\mathfrak{2}(u f e n t h a l t$ nidjt ermitteln oder fant man nidyt vom ŞaนEflux ober non Der Ireppe aus, fondern nur Durd) Den (Eingang Der $2 \mathfrak{B o h n u n g}$ in Die Burjuentube yelantigen, fo flingelt oder flopft man an Der Eingangsthür

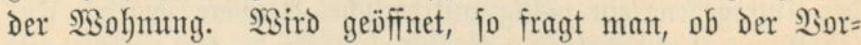

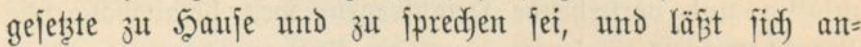
melden. Sit dies gejd)ehent und hat Der Diener die $\mathfrak{s}(1 \mathrm{f}=$ forderung zum Eintreten in ein bejtimmtes Simmer über= bradyt (weldyes er aud zugleid) öfrnet), jo tritt man ofyne 2Betteres ein. Sit Der Borgejebte jodjon Darit, fo madjt Der untergebene Difizier, die Fopfbedecfung it Der Şand, beim (Eintreten eine furge militürijd)e $\mathfrak{B e r b e n g u n g ~ u n d ~ t r i t t ~ j o ~}$ weit vor, daj der Borgejeşte ifnn deutlic) verjtehen faum, aber nidjt ju nahe herant. Sit im Simmer Niemand, jo Darf man nid)t Darin fid) etwa niederjetzen (Das wäre jebr

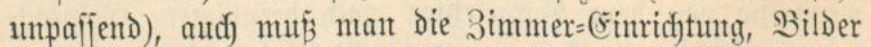

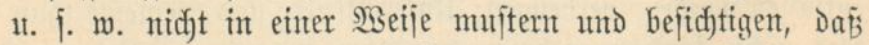
Der etwa plöblid eintretende Borgejetzte dies bemerfen fünnte;

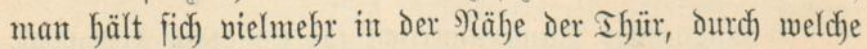
man eingetreten ift. Ilnteroffiziere und (jomeinte nehment im 3immer des Borgejebten Den 5elm nicmula von felber 


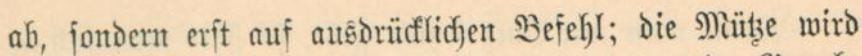
berettes nor der Ihür abgenommen, ment man meder Bjemehr nod) Seitengewehr bei fid) hat, mohingegent Der ïberbaupt bemaffnete Soldat die Miübe auf dem Ropfe behält.

Wiro man vom Borgejestent entlafien, fo madjt man als Dffizier eine furze Berbeugung, geft zur Ihüre, öfint bieje, verbengt fidd nodjmals gegen Den Borgejetzten, geht Gintaน und jd)liest die Ifür. Interoffiziere und Siemeine

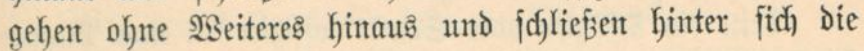
Thüre.

Der fitzento untergebene erbebt fith), um vorbeigehenden Borgejesten 5̧onneur zu ermeijen; Dffijiere Yegen die \$̧and an Die Fopfbedefuntg, Mamnjaften ftehen nur ftifl. Bu

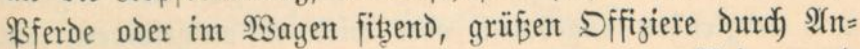
legen Der Şand an die Siopfbedecfung; Unteroffiziere und

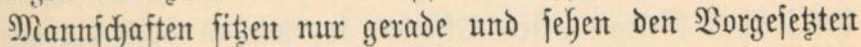
an, ohne mit Der ફ̧and zut grüjęen.

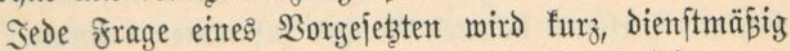

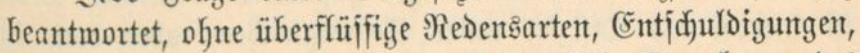

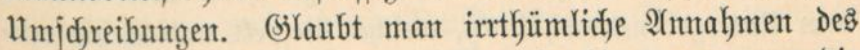
Borgejebten beridjtigen z̧ müffen, fo bittet man um die Erlaubniti, zu jpredjen, jobald der Borgeję̧te geendet hat, fällt ifm aber niemals in bie Rede.

Wird man von einem Borgejeştent gerufen, fo ant=

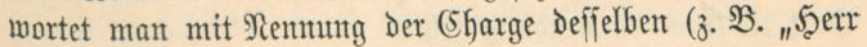
Stabsarzt" ${ }^{\prime \prime}$ und eilt zut ifm hin.

Begegnet man auf ber Ireppe, in Der Ihür oder fonjt an einter jajmalen Faffage einem Sorgejetsten, fo tritt mant zur Eeite und läf̆t ifn vorbei, indem man die Front nad) ifm hin nimmt; war man erft menige Stufen Der Ireppe hinauf= oder Ginabgeitiegen, jo tritt man auf Den Ireppen= abją̧, bezügliç ซেlux zurüđf. 


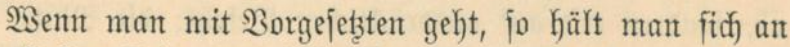
ihrer linten Seite und läbt ifnen beim Durdugange Durdf Thüren ftets dent $30 r t r i t t$, nadjoem mant ifnent die Thür geöfintet.

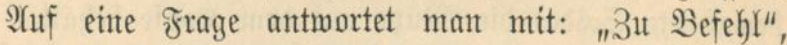
went man fie bejaht; mit "Nein" unter Nentung der (Sharge,

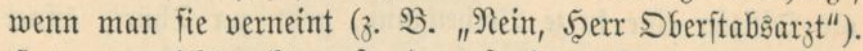
Fat mant nicht redjt veritandent, fo fragt mant 3 . B. "פSie

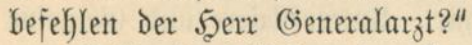

Iritt ein Dffizier oder Sanitätsoffizizer in eine Mant=

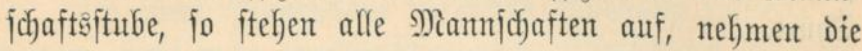
Ffeife oder (Sigarre aus dem Munte und die Meütse vom Sopfe, went fie biejelbe etwa aufgejetz̧t hattent.

Erthält Der Untergebente vom $\mathfrak{B}$ orgejeţtent eine Sigarre und Dergleid)en angeboten, oder wirb er aufgefordert, ein

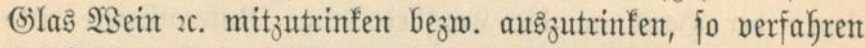
bei foldden (jelegentheiten Difiziere jo, wie es audy bei an= Derent Ständent Der guten Biejellidjaft Sitte iit, wenn mant

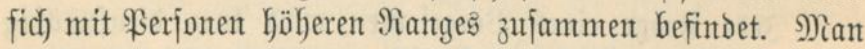
ift hierbei als Dffizier unter Seintesgleidyen, aber Dody in

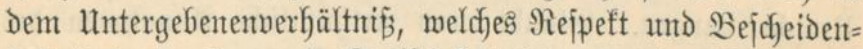

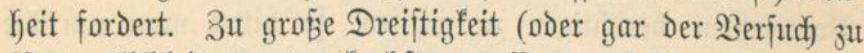
Bertraulidjfeitent) wäre hödjft umpajfent. Bejtimmte Siegelnt

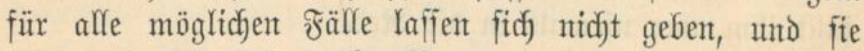

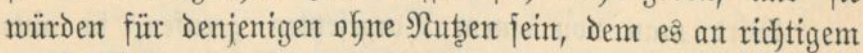

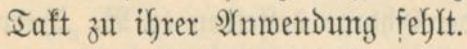

Interoffiziere und (siemeine jollen fidy bei foldyer (jie= legentheit nidjt bedanfen, jontoen die (sabe jujweigento in Diențtmäpiger Salturg antuehmen, Bietrü̈te follen fie auf

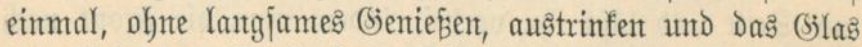
Dant feitwörts (Fenfterbrett, Iijd) niederjełzen; Die Cigarre

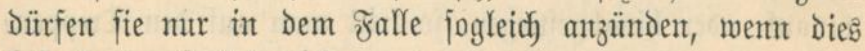
bejonders gejtattet wiro. 


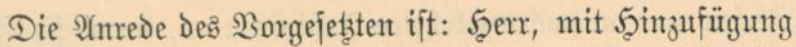

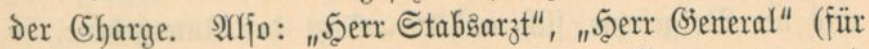
(Sieneralmajors); "(Excellenz" für (Sienteralliententants unto böhere (Shargent. Ferjonen von füriftlidjer (Sieburt redet mant

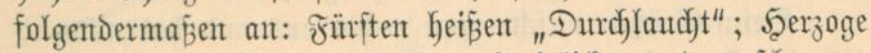

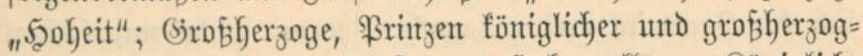

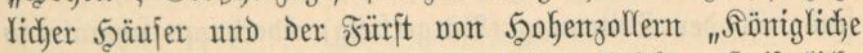

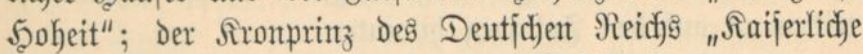
Şokeit"; Der Raijer jowie Rönige "Majeftät".

Sffiziere, Santitätg̊offiziere und obere Militärbeamte madjen Front vor Dem Saijer und Der Raijerin; Die prentiji=

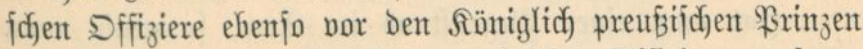

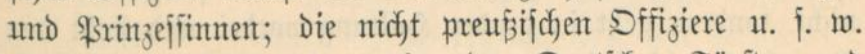
madjen Front vor Den regierenten Dentichen Fürjtent und Deren (Semahlinmen inmerbalb Deren Sandes̊grenzen. Beim Frontmadyen wird Die 5̧and an Die Ropfbedecfung gelegt.

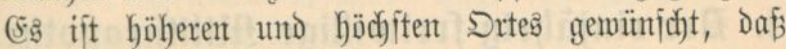
zmijd)en Den oberen Beamten einerjeits und den Dffizierent andrerjeits eine Begrüfuntg ftattfindet. (Eฐ gejdjieht Durd) Nulegung Der 5anto an die Sopfbedecfung. Eine itrifte $\mathfrak{B} e r=$ pflidytung befteht nidyt und fommen mux Die Şüflidjfeits:

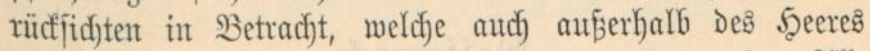

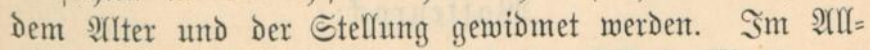
gemeinen läpt fiḑ) behaupten, Dá ein gebildeter Nant, nor

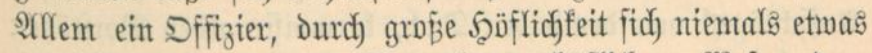

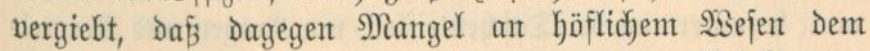

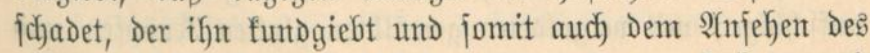
ganzen Standes, Deffen Uniform man trägt. - ־abnen unঠ Standarten finto wie Borgejeţte zu grüfent.

Unteroffiziere und Mammjaften nebmen eine dienjtliche Scaltung, b. h. möglidyjt grade Rörperbaltung mit anliegen= Den geftredtten $\mathfrak{A}$ rment, Durd)gedrüiften Finteent, gejd)lofientent

5onmettr Der Dffizitere Pinsiere

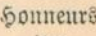
ber uebrigen 


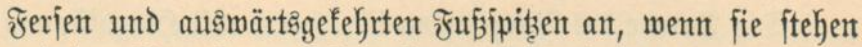

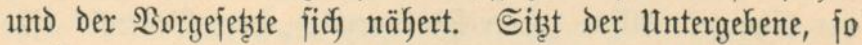
mutí er auffitehen. Im

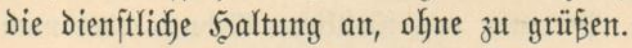

Beim Grinfent meidjt mant red)ts auts und hat nament= lid) Die Trottoirs frei ju madjent, went joldje mur fdumal find; ift Der Seitentweg (Bürgerfteig) überbaupt jojmal, jo tritt mant auf Dent Fahrweg himunter.

(Es ift Frout zat madjen vor Dem Saijer uto der Saijerin, dem Randesherrn utto feiner (Siemahlin und den Frinzen anto Srinzeffinten des regierentent 5̧aujes.

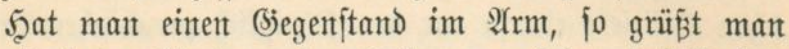
nidjt, fondern geht in grader Şaltung vorbei unto fiegt dent Borgefeţten bierbei an.

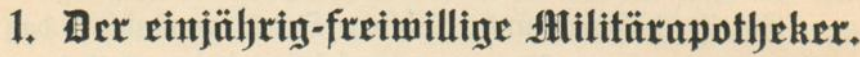

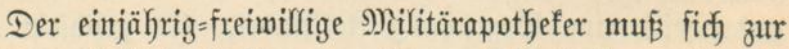
befohlenten Beit in Uniform meldent. Die Utriform ift fols gendermafen bejdaffen:

\section{Siaffentod:}

Hniform

Bon dunfelflautem Iudd mit Siragen unt jdjwedijaden

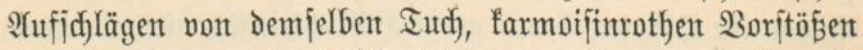

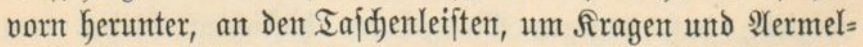
aufidjläge; mit vergoldetent gemölbten glatten Snẗöpen und Id jelflappen von farmoifinrothem Iudd mit einer Edjutr in Den Randeşfarbent eingefá̧t.

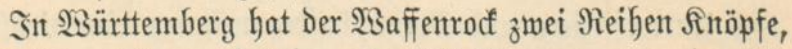

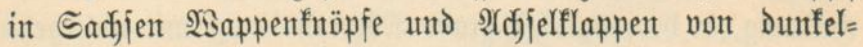
blauem Iudd und in Bayernt verfillberte glatte Sitüpfe. 


\section{Beinfleider:}

Iutubeinfleider der Infanterie $=$ Dffiziere mit farmoifint rothen Boritöfen an Den Seitentühten; in Bayern von der

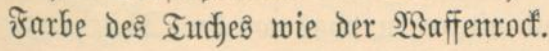

$$
\text { Selm: }
$$

Rederbelm mit edfigem $\mathfrak{B o r d e r}=$ und abgeruntetem Şinter $=$

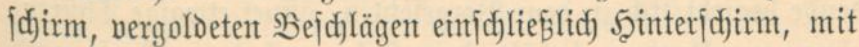
glatter Spibe auf vierblättriger Unterlage, Geraldijđjem $2(b l e r$ (ohne Devijenband) mit Dem গamengange F. R., Landes= fofarde und vergoldeten fonweren Sd)uppenfetten; in $\mathfrak{W u ̈ t t e m =}$

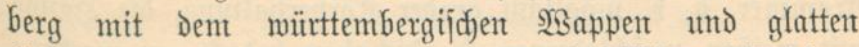

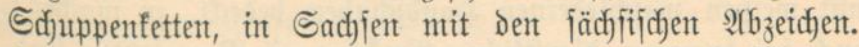
In $\mathfrak{B}$ ayern: Infanterie = Dffiziershelm, aber mit verfillbertent Bẹj)lägent.

\section{Degen:}

Irüherer Infanterie $=$ Dffizierg $=$ Degen mit Fortepée von Biold und dunfelblauer Seide.

\section{Mit täe:}

Bon Dunfelblautem Iud mit farmoifinrothem Borftó

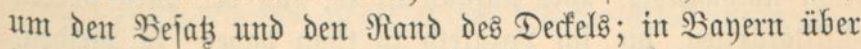
Der fofarde einten verfilberten heraldijdjen $20 ̈$ ment.

\section{Mantel:}

Bon Dem (Siruntutud) und naर्d) Dem Sdynitt der Miäntel bezm. Faletoţ Der Snfanterie= Dffiziere mit dent gleidyent finöpfen wie am $\mathfrak{R}$ affentod und Dunfelblauem Iuthfragen mit farmoifintotben Borftöfien und Sdyulterflappen wie an $\mathfrak{B a f f e n t o f f . ~}$

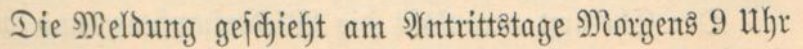
bei dem Chefarzt Des (Sarnifonlazarethy unt dem Boritand der Dijpenfiranftalt. Sind Beide im \&azareth nidft ant= 
mejent, fo nimmt der madythabende $\mathfrak{A}$ rat die Mieldung vor= Yäufig entgegen und übergiebt bie Dijpenfinanjtalt vorläuftg, wo nidjt mehrere Nilitärapothefer fint. Sobald der Shefargt

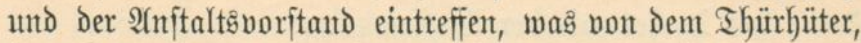
Dem jogenanten $\mathfrak{B o l i z e i = 1 4 n t e r o f f i z i e r , ~ a u f ~ B e i t e l l u n g ~ m i t = ~}$ getheilt mird, ift die Mieldung zit wiederholen. Der Mel= dento begiebt fich zu Dem Sorgejebten, öffnet etwaige Ihüren, ofne anzuflopfen, behält Den Jeelm auf Dem Siopfe und

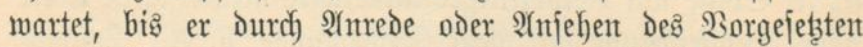
zur Meldung aufgefordert wirb. Die Meldung ift it ftrammer, ঠ. h. möglidfit grader Rïrperhaltuntg, die 5̧änte mit meifien majd)ledernen Scandjdubent bedect, zu madjen; aud bei fernerem dienftlidjen Berfehr mit Borgejeb̧tent fint die Regeln zu beadten, die oben für Interoffiziere unt Mannjd)aften gegeben find. Die Meldung lautet: (Einjül)rig= freimilliger Mitlitärapothefer Soundso meldet fid zutm Dienjt= antritt.

Die Meldung ift aud beim (sarnifonarzt und bem Sommandanten, bezügliody dem Sianijonälteiten abzuftatten.

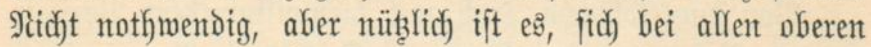
Mitlitärärztent, die im $\mathfrak{L a z a r e t h}$ zu thun haben, zu melden.

Die auf den (Eintritt des Mitlitärapothefers bezüglidjent Sittheilungen an die betreffenden Bebörden und fonjtige Errfordentiffe merden, abgejehen von dem Rebentalauf, vom (5) hefarzt bejorgt.

Der Rebentalauf ift vom Militärapothefer oreimal zu jadretben, und find dabei die Regeln zu beobadjten, die obent

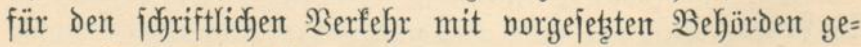
gebent futd (ङ. 22 f.).

Der Chefargt veranlapt die $23 i e d e r i m p f u n g$ und beant= \$ereibisung tragt die Bereibigung des Neuteingetretenten. Diejelbe ge=

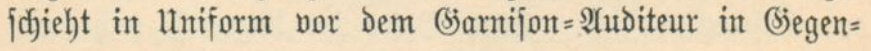




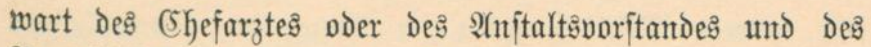
Frotofollführers, indem der Edjwörende mit dem Şelm auf

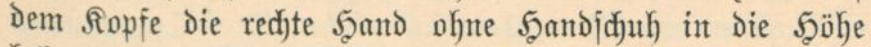
bebt und den (Fid ablieit oder nadjpridyt. Der (Fio lautet

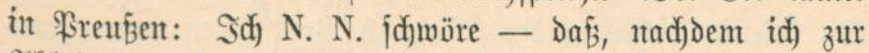

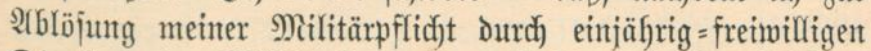

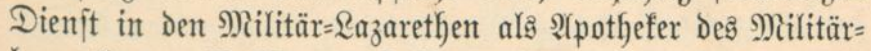
lazareths zut N. N. angeitellt morden bin, idj zuvörderft Seiner Majeftät Dem Rönige von Rrenjen, meinem Sfller= gnädigiten Randeshyerrn, will tren, geborjam und ergeben jein,

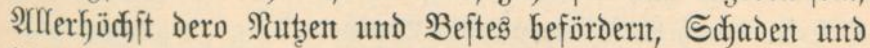
Radytheil aber abmenden will.

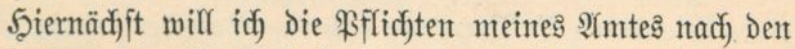
Borjadriften Der 2 (potheferfunjt und inşbejondere der mir

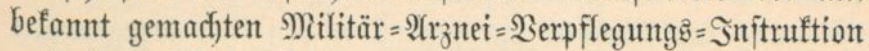
unt Der diejer gefolgten Injtruftionen unverbrüd)lidj be= obadjten, Die Befeble und Anoronungen meinter Borgejetstent

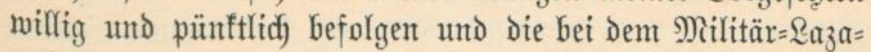
rethoienit fo nothmendige Subordination nie au Den Natgen jetzen, Die $\mathfrak{A}$ ranteimittel gehörig und mit Sorgfalt anfertigen,

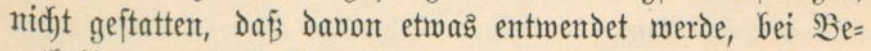
untheiluntg und Friffutg Der gelieferten und vorbandenent

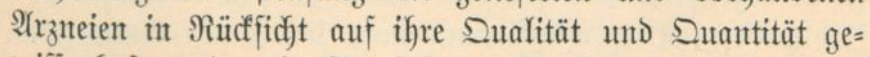

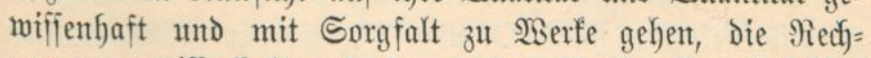

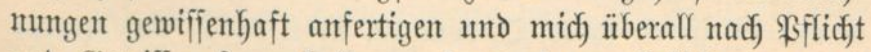
unto Ssewiffen fo verhalten, wie eร einem ebrliebenden und red)tjd)affenten 2 (pothefer zufommt.

So mahr mir u. i. w. je nad Befenntniff.

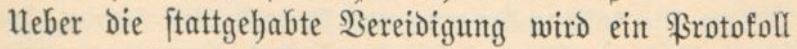
aufgenommen, Das Der Bereidigte unterzeidynet.

Der einjährig=freimillige Millitärapothefer gebört nady Der Rangordmung, weldje oben (ङ. 10 f.) angefübrt ijt, zu 
Den unteren Militärbeamten im Range Der Maunjuaften vom F̌elomebel abwärţ. Sn Folge defien ift ex verpflidjtet,

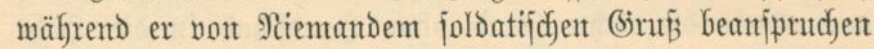
fant, Difiziere uno Sanitätsoffiziere zut grüfient. Dent it

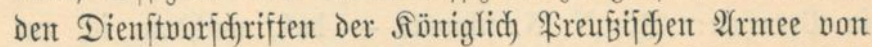

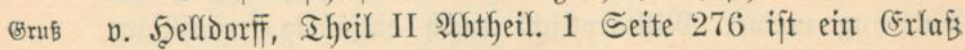

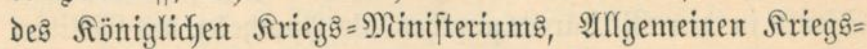

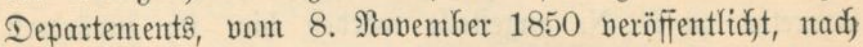
weldjem jeder Sillitär=llnterbeamter, weldyer feintent bejtimmtent Sititiärranty hat, went er fid in liniform befindet, jedent Difizier Durdy SAfafien an die Sinpfbedecfung zu grüfen hat.

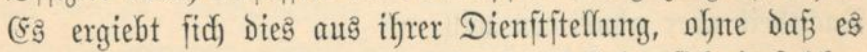
einer bejonderen Bejtimmung bedarf. Befindet fidf ein joldfer Mititïä=Unterbeamter nidyt in Untiform, fo hat er einte $\mathfrak{B e r}=$ pflidftutg zum Grïjen unt gegenüber Dent ibm begegnendent Borgejetstent.

Ueber Daร (Sin̈ben der oberen Millitärbeamtent jagt die

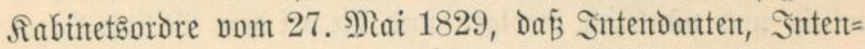
Dantuträtbe, Sutoteure in uniform und Militürprediger int Snat vont Dent Ilnteroffizieren, Epielleuten uto (Siemeintent

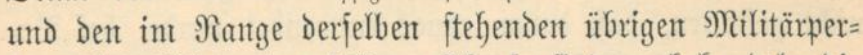
jonten begrübt merden jollen. EEbenjo fint zu bebandeln die 3ablmeifter (Sriegsminifterium vom 10. Suni 1854), For=

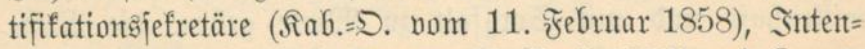

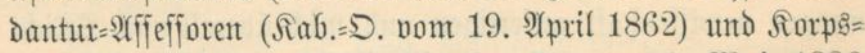
und Sberrobärzte (Millit. $=$ Beterint. $=$ D. vom 6. Mai 1886 $\left.\S 6,2) .{ }^{*}\right)$

*) Die Uniforn ber genannten Beamten ijt folgende: Dunfel=

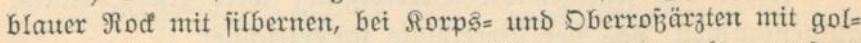

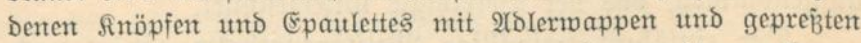

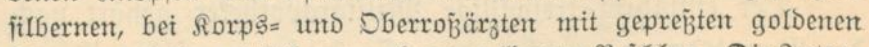

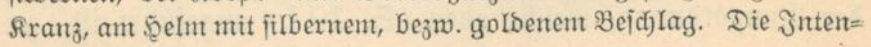


In Diฐziplinarer Beziebung itehen Die Nilitärapothefer Dişiprinar=

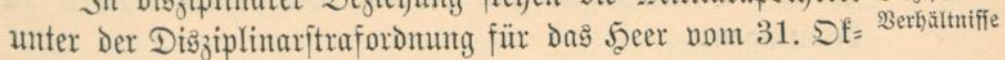

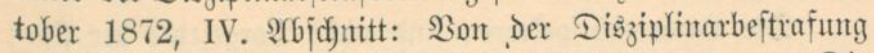
Der Mitlitärbeamtent. Der $\$ 32$ diejes (jejebses lautet: Die

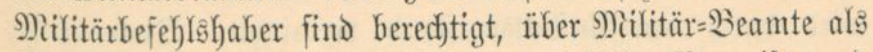
Disziplinarftrafen $\mathfrak{B a m u n g e n ~ u n d ~ e i n f a d j e ~}$ Bermeije und über untere Sitlitürbeamte Die gegen Unteroffiziere, weldye

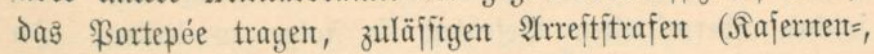

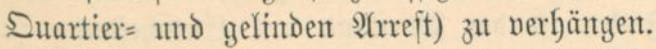

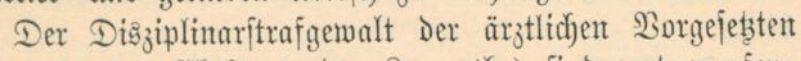
(in specie der (5hefärzte der $\mathfrak{L a j}_{j}$ arethe) find unterworfent: die \$harmazenten 2 . (\$ 17 Der $\mathfrak{B e r o r d m u n g}$ über D. Drg. D. San.=凡. vom 6. Jebruar 1873).

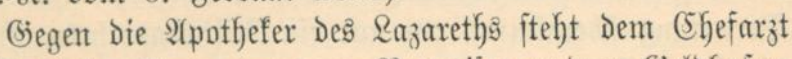

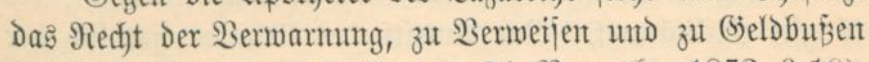

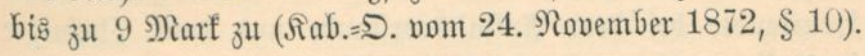

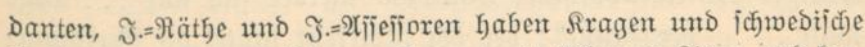
2(uff́)läge von Dunfelblauem Sammt mit fitlbernen Siţen unto far=

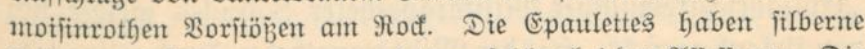
FelDer und filberne Franzen; Ieb̧tere feblen bei ben 2 (nīelïoren. Die Adfjelitüdfe find gleid) Denjenigen Der Stabsoffijiere, aber mit blau Durchflod)ten; 2(ijefïpren haben mut cine Silbertrejie mit farmoinfin= rothen Utrteriutter und Dem 2tslerwappen.

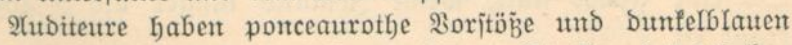
Rragen und 2 Aufid)läge mit jilbernen \&iß̧en. Die Epaulettes haben Felber von Dunfelblauem Tutd).

Die 3ahlmeijter haben weiğe \$oritöb̆e, weiñe Felder in ben

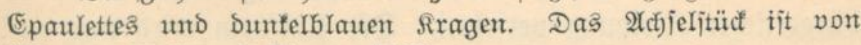
filberner Irefie mit $\mathfrak{3}$ appenjujilb.

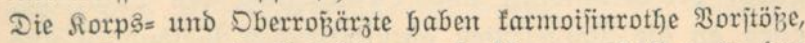

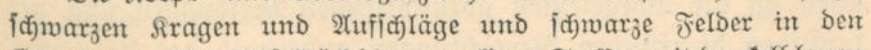

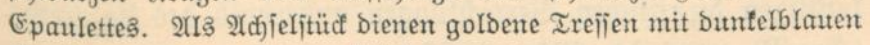
Streifen und bem $2 a p p e n i d j i l b$. 
Weitere $\mathfrak{B}$ eftrafungen find bei Dem forpş=(Sieneralargt zu beantragent.

Die Razarethinjpeftoren find nidjt Borgejetzte der $\mathscr{A p p}=$ thefer, meil fie Cinilbeamte fint. Der dienjtlidje Berfefrr

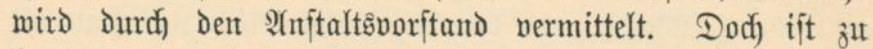
bead)ten, dafis fie oft Hebermittler von Befehlent des (5hef= arjtę oder fiommandanten bezm. (Sarnijonälteiten find; joldjen ïbermittelten $\mathfrak{B} e f e b l e n t$ ift Folge zul leiften. In żweifellfaften Fällen ift der Borjtanto um Entjudjeidung zu bittert.

UrIaub

Der Militärapothefer hat mie andere (Einjührig=ðreimillige Anjprudy auf 14 Iage Urlautb, weldyen er bei Dem Borftand Der Dijpenfiranftalt beantragt und vom Shefarzt erbält. Die Bertretung ïbernimmt, wo nur ein Mitlitärapothefer iit, der

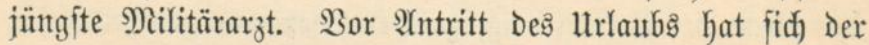
Beurlaubte bei dem Anftaltênorftande und dem Chefarjte

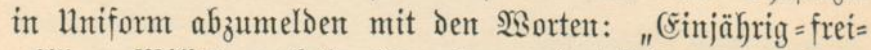
williger Militärapothefer Soundso meloet fidú auf ... Iage nad) ... . beurlaubt"; bei der Rüuffehr lautet die Mieloung: „Ëinjährig=freimilliger Militärapothefer Soundso meldet fiḑ von lurlaulb zurüđ̆". Şat der Millitärapothefer, Durd) zwingende (Siründe veranlafit, länger als 14 Iage Urlaub gehabt, jo

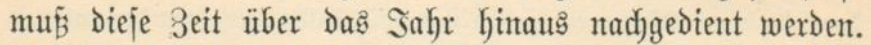
Daffelbe fundet ftatt, went Jemant länger als 8 $\mathfrak{1}$ odjen frant i ift.

Unifornt. swang

Der täglidje Dienjt in Der Dijpenfiranjtalt miro, aus= gentomment in Sadjjent, wo ftets Untiform getragen wirb, it bürgerlidjer flleidung verjeben. Die Uniform wird mur an= gelegt bei Revifinten und Befidjtigung Des $\Omega_{a z a r e t h}$, bei allent an Die Borgejesten abjujtattenten Mieldungent und bei

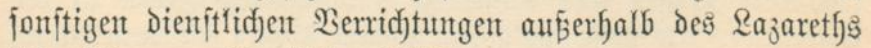
(Fir. M. vom 24. Mä̈rz 1877). 
Der (sieja)äftâbetrieb in Der Dijpenfiranitalt wird nad Den erlaffenten Inftruftionen und Beroronungen gefüfrt.

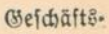
betrieb

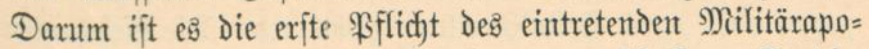
thefers, fidj) Renntnifi von Demjelben zu verjajaffen. Da die= jelben fid) in jeder Dijpenfinanftalt befinden, jo jollen die= felben hier nur in ifrent 5̧auptpunften erwäbnt werden.

Der einjährig = freimillige Millitärapothefer ijt für die

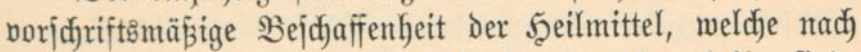
der Pharmacopoea Germanica ed. altera zu beurtheilen finto, und Die ridjtige Bereitung Der dijpenfirten $\mathfrak{A}$ rzneien verant= wortlidd.

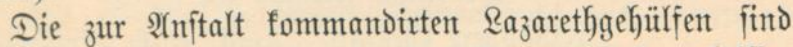

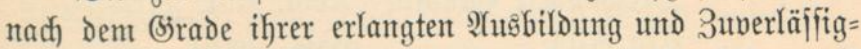

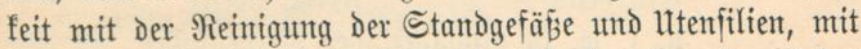

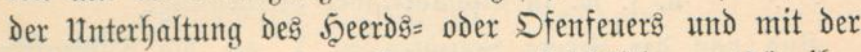

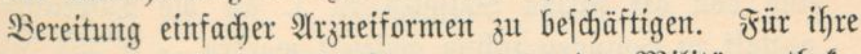
pharmazentijd)en Şülfäleiftungen trägt Der Millitärapothefer Die Berantwortung. Die Razarethgehülfen find Dem Militär= apothefer untergeorontet. Etwaige Ingehörigfeiten fint Dem

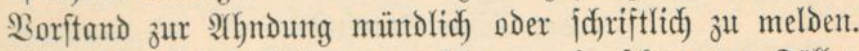

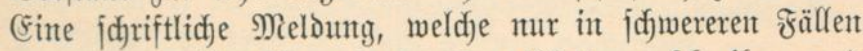
anzumenden ift, ift auf ein Duartblatt zu jdgreibent und mürde z. $\mathfrak{B}$. Iauten:

$$
\text { Drt, Den . . ten Mionat } 18 \ldots
$$

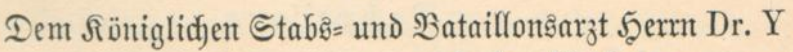
ફॄod)mohlgeboren

\$ortfaut einer [d)riftlid\}en Meloung

Die Meldung, Dá̧ Der $\mathfrak{L a}_{3}$ arethgefüllfenlehrling Soundso heute ofne Entidjuldigung Dent Dienft in Der Difpenfir= anftalt verjäumt hat.

\section{$\mathrm{Z}$.}

Einjührig=freimilliger Militärapothefer. 
Beidiaffung

ber

2rzneien

Die $\mathfrak{A}$ rzneimittel, 9) Caterialmaaren und Rebenbedürfniffe

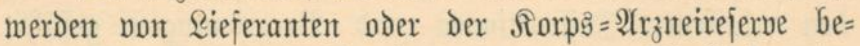
zogen oder Durd Selbftbereitung gemontent. Die Rieferantent beftimmt die Intendantux und die $\mathbb{R} a_{3}$ arethverwaltuntg, weldje

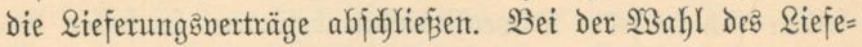
ranten ijt möglidjite Centralijation zu erjtrebent.

Die Beftellzettel werden vom Ehefargt, Boritand und 9)ilitürapotbefer unterjdrieben, z. $\mathfrak{B}$.

Beftellzettel

$$
\text { Drt, Dent . . Mionat } 18 \ldots
$$

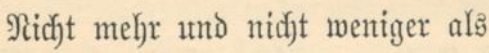

Dreifundert (jramm Acid. sulf. pur.

Ein Sillo

Bals. peruv.

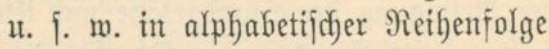

fint bon ber Firma Soundso zu $\mathrm{Y}$ an bie Dijpenfir = anfitalt zu Soundso ridjtig geliefert worden.

$\mathrm{X}$.

Shefarzt.
Y.

Z. গad). weifungen

Die anfommentent 2 Saarent werdent in fogentante "Nad $=$

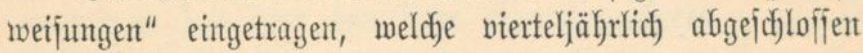
merden:

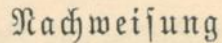

über bie im Raufe Des 1. Sierteljahrs 1888/89 von Der Firma Soundso jut $Y$ an die Difpenfiranjtalt des Röntig= lidjen Bamifonlazareths zu Soundso gelieferten $\mathfrak{A}\left(\mathfrak{z}_{z}\right.$ nei $=$

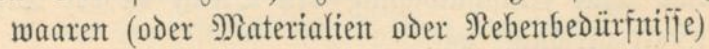
dent ... ten Mionat 18 . .

Dreifuntort (Bramm Acid. sulfur. 
1. Der einjährig=freinillige Militärapothefer.

Ein Sillo

Bals. peruv.

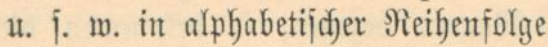

Y.

Z.

Stabsaarzt und Borftand.

Mititïrapothefer.

\section{Den . . ten Minat $18 \ldots$}

Fünffundert (Brantm Acid. hydruchlor.

น. โ. w. wie obent.

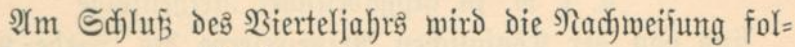
gentermajen abgejoldofien:

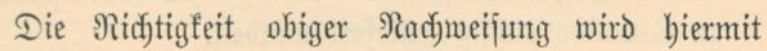
bejodjeinigt

Drt, Den . . ten Mionat $18 \ldots$

$\mathrm{X}$.

Ehefargt.

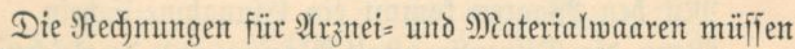
von Der Dis̄penfiranftalt bejcheinigt werden in folgender $2 B e i j e:$

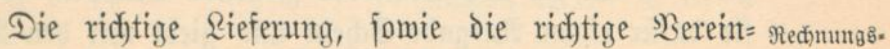
nabmung in die tabellarijd)e Medifamentenbered)mung pro I. Bierteljafbr 1888/89 wird Giermit bejujeinigt Y.

Z.

Stabąarzt und Borjtand Militärapothefer. Der Dijpenfiranitalt.

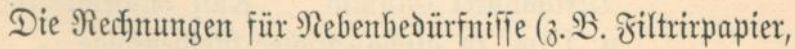
תartenblätter น. j. w.) werden folgendermafien bejdyeinigt:

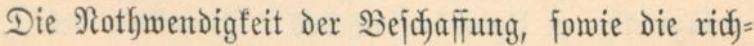
tige Rieferunty wird hiermit bejodeinigt.

Y.

Stabsిarzt und Boritand Der Dijpenjiranitalt. beidei:

nigung 
Werden non Der Sorp: $=\mathfrak{A}$ răneirejerve oder anderen Beförden : $3 a a r e n$ bezogen, jo befonmen diejelben einen $\mathfrak{A}$ taggabe= Sdyein folgenter $\mathfrak{A}$ rt:

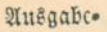
Edhein

Eimabme: Ed)ein

$$
\mathfrak{A} \mathfrak{u} \mathfrak{g} \mathfrak{a} \mathfrak{b} e=\subseteq \mathfrak{d}) \text { ein }
$$

গidjt mehr und nidjt mentiger als $\begin{array}{ll}\text { Jünf } \mathfrak{T} \text { İlo } & \text { Empl. adhaes. } \\ \text { Drei fillo } & \text { Glycerin }\end{array}$

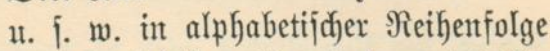

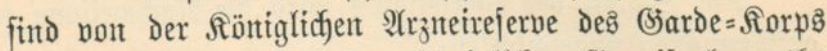

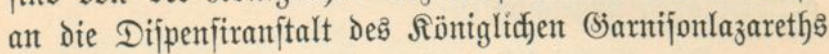
zut Soundso ridjtig geliefert wordent.

$$
\mathrm{Y} \text {. }
$$

Z.

Stabęarzt utd Borjtand.

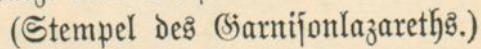

Mit Den $\mathscr{B}$ aaren fommt ein (Eimahme=Sdjein.

Went die Dijpenfiranftalt $\mathfrak{B}$ aarent und Dergleidjen $a \mathfrak{b}=$ giebt, 子. $\mathfrak{B}$. an in': Stantöner ziehende Iruppent, jo erhält fie dafür einen $\mathfrak{Y}(1+9) g a b e=S d j e i n$ und giebt mit den $\mathfrak{W a a r e n}$

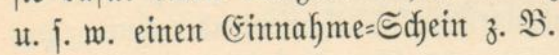

\section{(Einnahme= 5 d)eint.}

গidut mebr unto nidft mentiger al:

$$
\text { Fünfzig Gramm Acid. sulfur. }
$$$$
\text { Dreifuntert Siramm Kali chloric. }
$$

น. ¡. m. in alphabetijadjer Reihenfolge

find von Der Dijpenfiranftalt Des Rëniglidjen Ssarnijon=

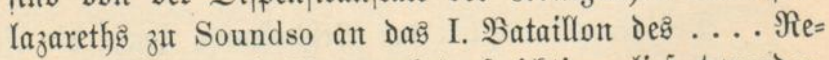

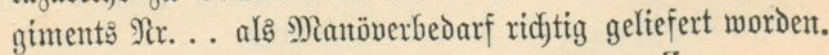
$\mathrm{Y}$.

Stabaarzt und Boritand Z. Der Dijpenjinanitalt. 
1. Der einjährig=freiwillige Militärapotheler.

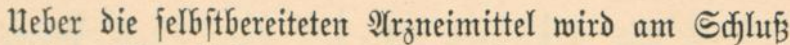

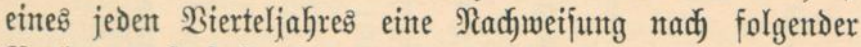
Borlage aufgeitellt:

\section{Nad)weijung}

über die im Raufe dę I. Wierteljahrs $1888 / 89$ in Der

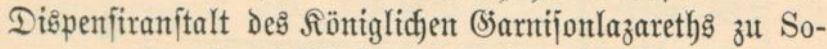
undso jelbit bereiteten $\mathfrak{A}_{\mathfrak{z}}$ mteimittel.

\begin{tabular}{|c|c|}
\hline $\begin{array}{c}\text { Benennty deร } \\
\text { Fräparats }\end{array}$ & Dazu verbraudd \\
\hline $\begin{array}{l}\text { Empl. adhaesiv. exten- } \\
\text { sum } 76 \mathrm{~m}\end{array}$ & Empl. adhaes. 3950,0 \\
\hline Inf. Senn comp. 300,0 & $\begin{array}{l}\text { Fol. Senn. } 37,5 \\
\text { Aqu. dest. } 275,0 \\
\text { Tort. natron. } 37,5 \\
\text { Manna } 75,0\end{array}$ \\
\hline
\end{tabular}

1t. ¡. w. in alphabetijd)er Reibenfolge. $\mathrm{Y}$. Z.

Stabsarart und Borjtand. Mititiärapotheter.

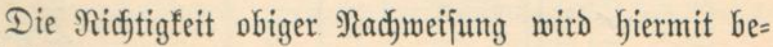
jodeinigt.

Drt, Den . . ten Monat $18 \ldots$

$\mathrm{X}$.

Ehefargt.

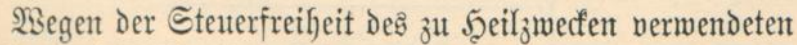
Spiritus mird ïber Den $\mathfrak{A} b=$ und 3ugang deffelben eine be= jontoere Radjweijung geführt: 
II. Die Seeresapothefer.

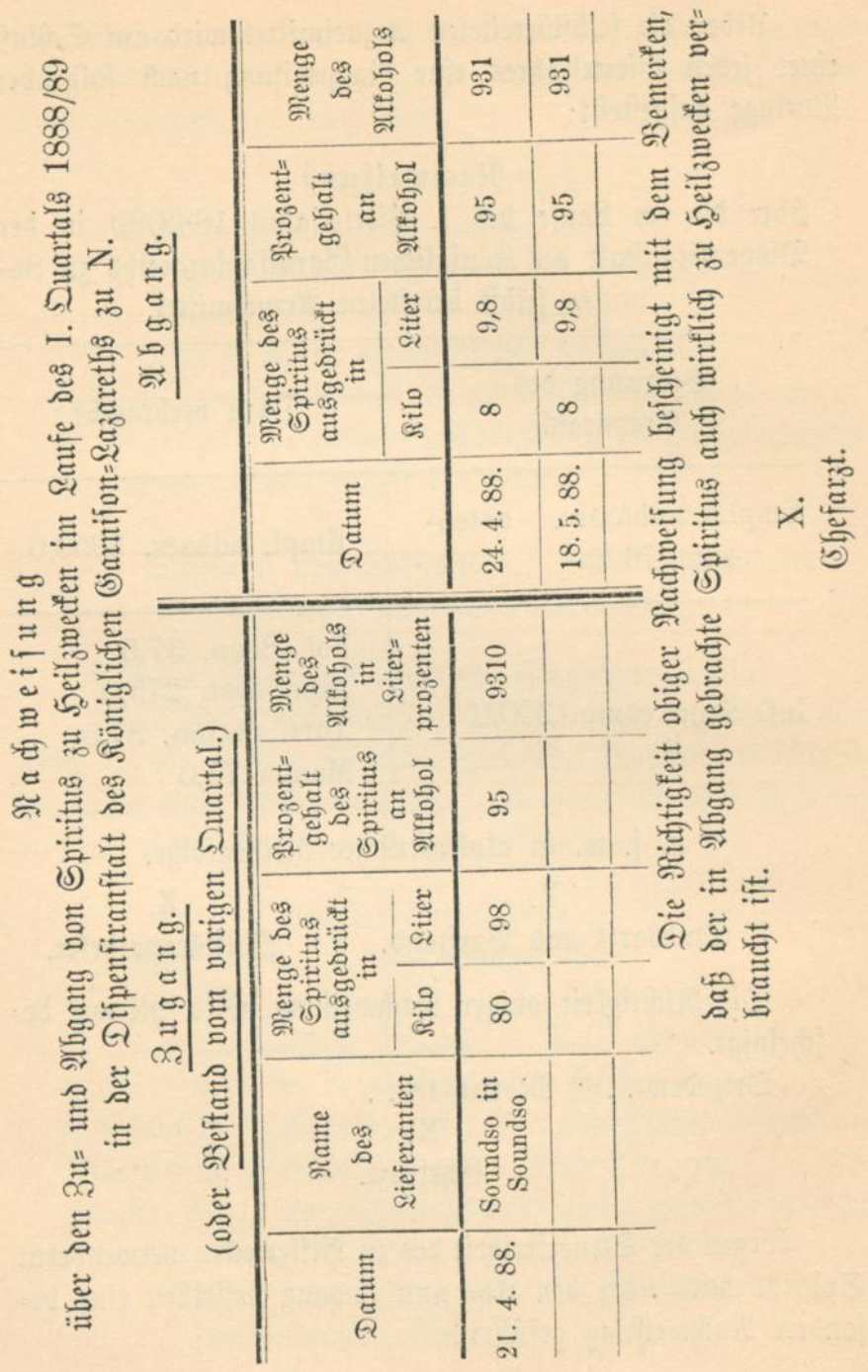


(Enolid) wirb aud über Den Berbraud) von Papier=

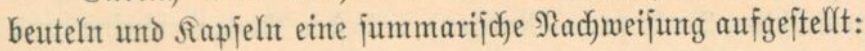

\section{Rad)meijung}

über die im Raufe Des I. Bierteljahrs 1888/89 in Der

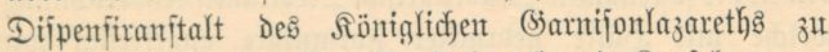
..... verbraudften Fapierbeutel und Siapieln.

\begin{tabular}{|c|c|c|c|c|c|}
\hline & $\begin{array}{l}\text { Beutel } \\
\text { Nr. } 1\end{array}$ & $\begin{array}{l}\text { Beutel } \\
\text { Rir. } 2\end{array}$ & $\begin{array}{l}\text { Beutel } \\
\text { Rx. } 3 \mid\end{array}$ & $\left|\begin{array}{l}\text { Beutel } \\
\text { Nr. } 4\end{array}\right|$ & Rapielnt \\
\hline $\begin{array}{l}\text { Beitand am } \\
\text { 1. } 2 \text { (pril } 1888\end{array}$ & 79 & & & & \\
\hline $\begin{array}{c}\text { Bugang int } \\
\text { 1. } \text { Bierteliafr } 1888 / 89 \\
\end{array}$ & 200 & & & & 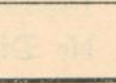 \\
\hline $\begin{array}{c}\text { Summa bes Beitandes } \\
\text { und Buganges }\end{array}$ & 279 & & & & $\cdots$ \\
\hline $\begin{array}{c}\text { Berbrautdjt im } \\
\text { 1. Bierteljafyr } 1888 / 89\end{array}$ & 179 & & & & \\
\hline Beitand an 1. Juli 1888 & 100 & & & & \\
\hline
\end{tabular}

..... Den 1. Juli 1888 .

Die Dijpenfiranjtalt. $\mathrm{X}$.

Y.

Stabsarzt und Boritand.

Militïrapothefer.

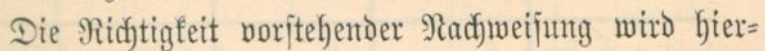
mit bejojeinigt.

Z.

(5hefarizt.

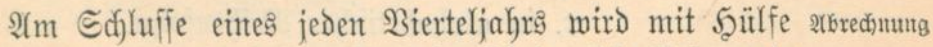
Der Nad)meijungent und der Einmafmejd)einte die Siejammt=

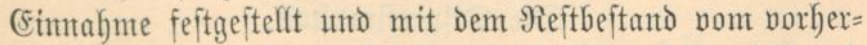
gebentent Bierteljabr vereintigt. (Sileid)zeitig wird auf (strund Der Berorduntgábüdjer, weldje an leţten Tage des Biertel= 


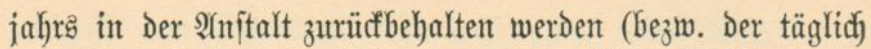
gemad)ten Berbraud) mit Berürffidjtigung Der $\mathfrak{B a ̈ g e v e r l u j t e ~ u n d ~ e t m a ~ v e r j o d u ̈ t t e t e r ~}$

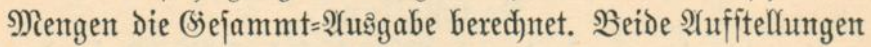
Gilden die jogenannte "Iabellarijaje Medifamentenberedynung", weldye mit Şülfe gedrutfter frormulare angefertigt wird. Derjelben werden aufier Den beident jodjon gentanten Belägent nod) zwei Heberfiduten folgender $\mathfrak{A}$ rt beigefügt.

\section{ueberjidyt}

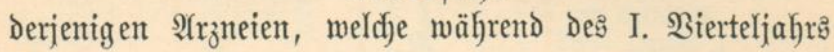
$1888 / 89$ von der $\mathfrak{A}$ rgneirejerve De: . . ten $\mathfrak{A}$ rmeeforps an

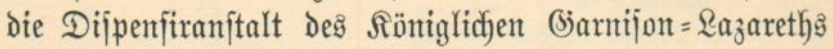
孔 $\ldots \ldots$ z morden finto.

\begin{tabular}{|c|c|c|c|c|c|c|c|c|}
\hline \multirow{3}{*}{$\begin{array}{l}\text { Benenmung } \\
\text { Der } \\
\text { Begenjtände }\end{array}$} & \multicolumn{6}{|c|}{ 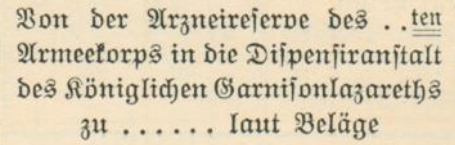 } & \multirow{2}{*}{\multicolumn{2}{|c|}{ Summa }} \\
\hline & \multicolumn{2}{|c|}{ I. } & \multicolumn{2}{|c|}{ II. } & \multicolumn{2}{|c|}{ III. } & & \\
\hline & Ko. & $\mathrm{g}$ & Ko. & $g$ & Ko. & $g$ & Ko. & $g$ \\
\hline Acid. carb. liq. & 6 & & 6 & & & & 12 & \\
\hline Beutel No. 1. & & $\begin{array}{c}\text { Stüt } \\
200\end{array}$ & & & & $\begin{array}{c}\text { Stüdf } \\
200\end{array}$ & & $\begin{array}{c}\text { Stüd } \\
400\end{array}$ \\
\hline
\end{tabular}

2c. in alphabetijoder Reibenfolge.

N., Den 1. Suli 1888.

Die Dijpenfiranjtalt. $\mathrm{Y}$.

Z.

Stabsarat und Doritant.

9)ilitürapotheter. 
Ueberjidut

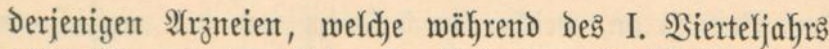
1888/89 aนเి Der Dijpenfiranftalt Des Röniglidjen (5ar=

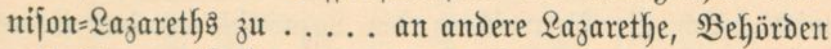
und Truppentheile zur weiteren $\mathfrak{B e r r e d}$ mung bei diejen abgegebent worden finto.

\begin{tabular}{|c|c|c|c|c|c|c|}
\hline \multirow[t]{2}{*}{$\begin{array}{l}\text { Benentutitg } \\
\text { Der } \\
\text { Begenjtände. }\end{array}$} & \multicolumn{2}{|c|}{ 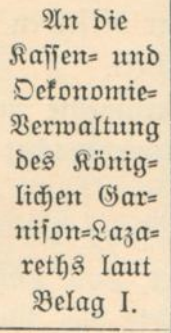 } & \multicolumn{2}{|c|}{$\begin{array}{l}\text { An ธaş } \\
\text { 1. Bataill. } \\
\text { 4. Earde= } \\
\text { Reg. 3. ₹. } \\
\text { Iaut } \\
\text { Belag II. }\end{array}$} & \multicolumn{2}{|c|}{ Summa } \\
\hline & Ko. & $\mathrm{g}$ & Ko. & $\mathrm{g}$ & Ko. & $\mathrm{g}$ \\
\hline Acid. benzoic. & & & & 10 & & 10 \\
\hline Acid. boric. pulv. & & 500 & & & & 500 \\
\hline
\end{tabular}

2c. in alphabetijuder Reibenfolge.

N., dent 1. Juli 1888.

Die Dijpenfiranjtalt.

$\mathrm{Y}$.

Stabsిarżt und Boritand.
Z.

Mitlitärapothefer. 


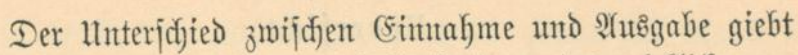

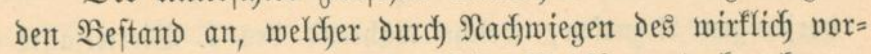
Gandenen fontrollirt wiro. Die Medifamententered)nung

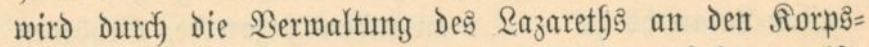
genteralargt gejdifft und Durdy den אorpştabsapothefer geprïft.

Die $\mathfrak{B e r b a n d s m i t t e l}$ werden, wie jujon oben angegeben, non Der Berbandmittelrejerve bezogent und in bejonterent Büdjern verjodriebent. Ueber diejelben wird ebenjo abgered $)=$ net, wie über Die $\mathfrak{A} \mathfrak{r}_{j}$ neimittel.

Die djintrgijd)ent Snitrumente und Deren Reparaturen merdent von dem Injtrumentenmadjer bejorgt, weldjem die Rieferuntg ïbertragen ift.

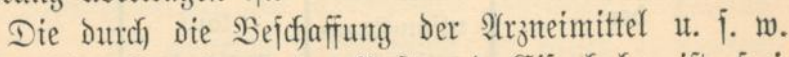
nothmendige Benutbung der Fojt unt Eijentabn ift frei, ment die Briefe und Pacfete Daร şort Militaria und dent Stempel des Razareths tragen. Die Jradytbriefe erbaltent als Franfaturvermerf Die Bemerfung: Die Beförberutg ge=

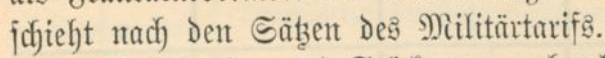

Die Sdjerbent uno Stü̈fe von jerbrodjenten (s)efäbent und

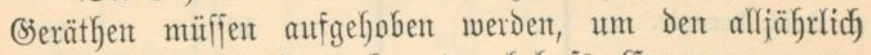
revidirenden Sntendanturbeamten bebufs Ernenterung vor= gelegt zut werdent.

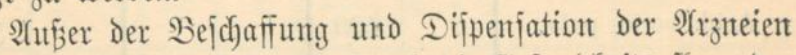
Gat der Mitlitärapothefer bie Durd) Die (jejundheits̊pflege der

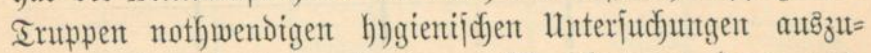
fübren, jomeit bie vorfandenten Eimridjtungen Daju aub = arinfwafier-reidjen. Fenter find in jedem Bierteljahr zehn Irinfwaffer=

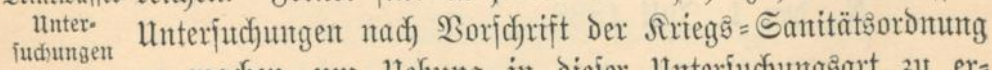
zu madjen, um Hebuntg in biejer Interjuddungånt zu er= langen. Ueber Dieje Unterjudfungen wird an Dent fiorps = ftabsapotbefer Berid)t exitattet nad) folgendem Sdjema: 
1. Der einjährig=freimillige Militärapothełer.

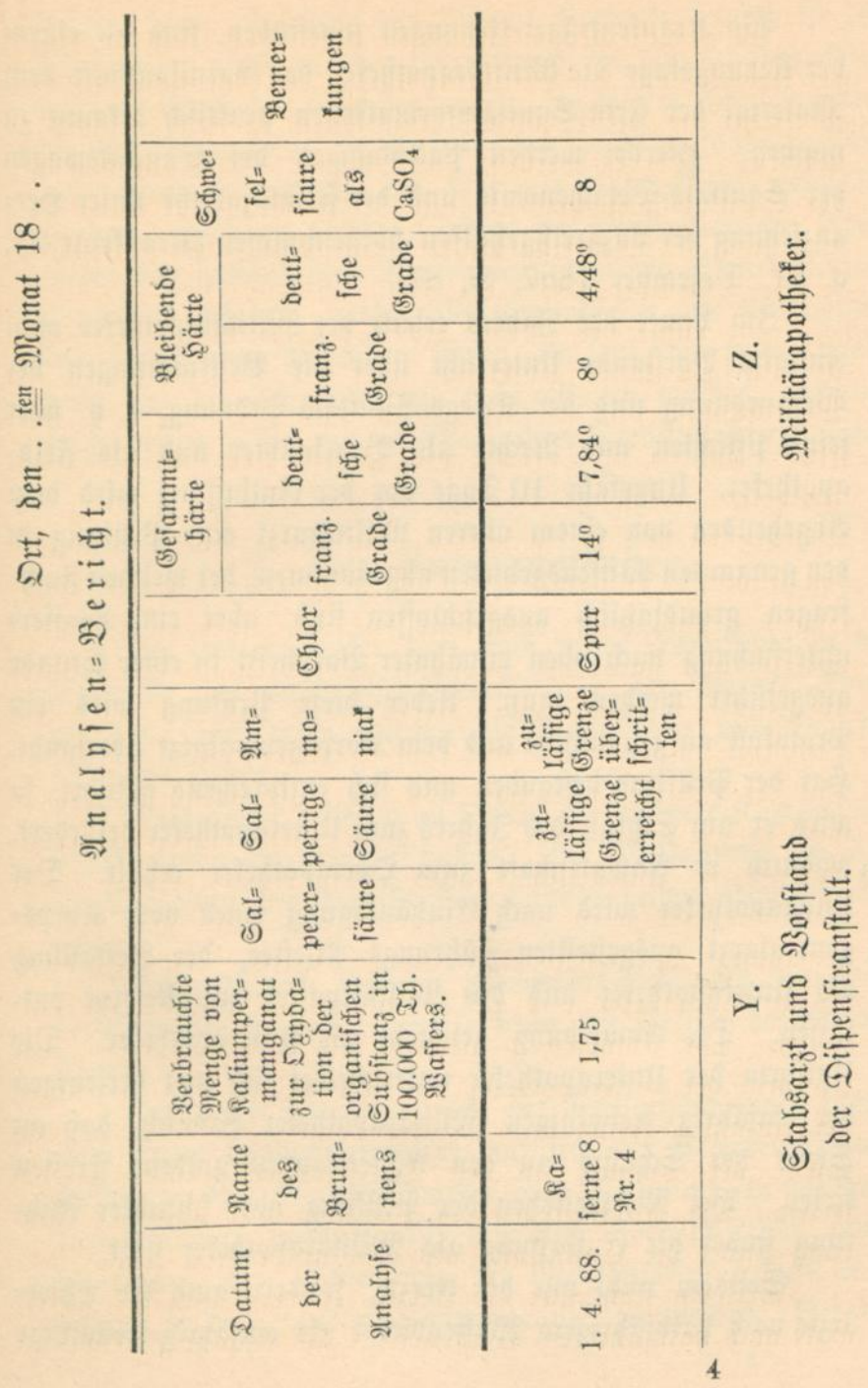


$\mathfrak{B}_{0}$ Rranfenträger= Hebungen itattfinden, finto an einem Der Ulebungstage die Militärapothefer Der Sarnijon mit Dem Material der Feld=Sanitätŝformationen praftijod) befannt jut madjen. Şierbei werden Facfübungen der Santï̈tâwagen Der Sanitüts=:Detadjements und Der Feldlazarethe unter Scer= anziehung Der $\mathfrak{L}_{\mathfrak{z}}$ arethgehülfen vorgenommen ( Frantentr.=D. v. 21. Dezember 1887, ऽ. 87).

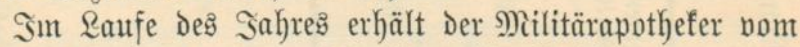
$\mathfrak{A}$ (njtalts $=$ Borftande Unterridyt über die Beftimmungen der

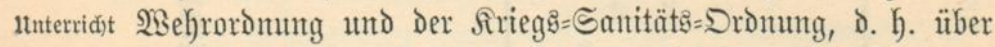
jeine Pflichten und Rechte als Beurlaubter und als Feld= apotbefer. Untgefähr 10 Iage vor Der Ëntlafiutg wird Dem Abgebenden von einem oberen Militärarjt einte Früfutg it

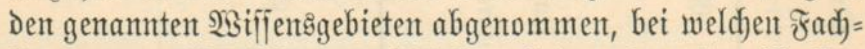

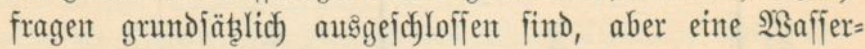
unterjudfung nadj) obent erwähnter $\mathfrak{B}$ orjadyrift in einter Stumbe

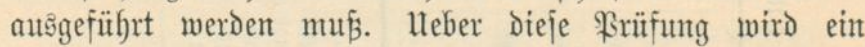

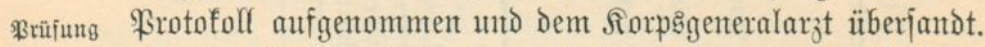
Sat der \$rüfling beftanden und fidf) entiprechend gefüfyrt, jo

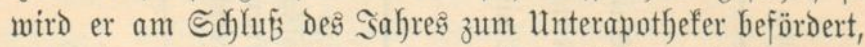
Beförberung wodurdf) ex $\mathfrak{A}$ (nwartjd)aft zum Dberapotbefer erhält. Der Unterapothefer wird nach Eintbändigung eintes vom forps:

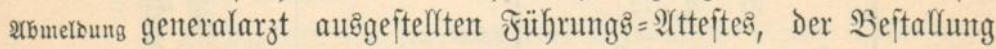
als Unterapothefer und des Millitärpaffes zur Rejerve ent=

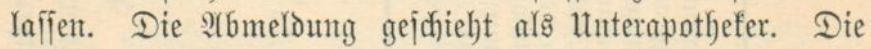
unter. Uniform Der Unterapothefer unterjdjeidet fid bon Derjenigen

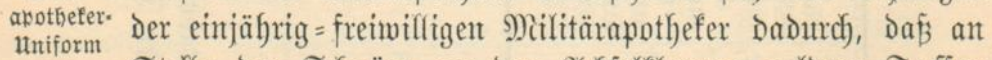
Stelle der Edyüre an den Iddjelflappen goldene Ireffen

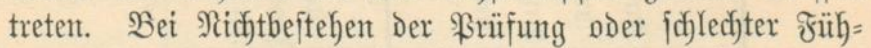
rung findet die Entlaffung als Militärapothefer ftatt.

Seitdem nidyt mu bie Serzte, jonvern aud die Ifier=

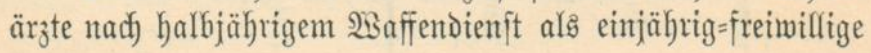




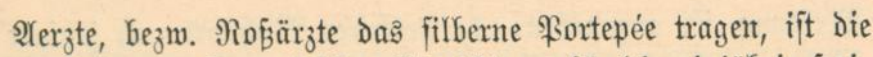

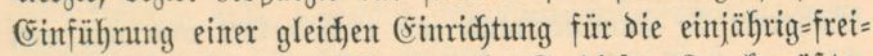

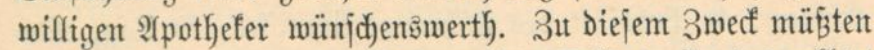

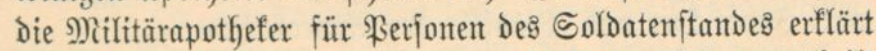
uto ifnen Der Rang Der \$ortepée=Unteroffiziere ertheilt

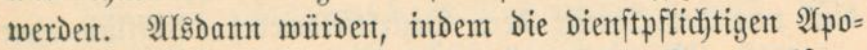
thefer den halbjäbrigen $\mathfrak{B a f f e n d i e n j t ~ v o r ~ D e r ~ S t a a t s ̌ p r i f f u t y ~}$ abmadłen mürden, mebr Sitlitürapothefer jur Serfügıng Der betreffenten Bebörden ftehen als jełst, wo viele zut alt find, um die Staatspprüfung redjtzeitig zu madjen, und in=

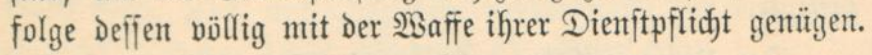

\section{Der Anterapotheker.}

Der Unterapothefer tritt nady) feinter (Entlafilumg it bie Sontrolle Der Randwebrbehörden, d. i. Der Bejinf $=$ Som=

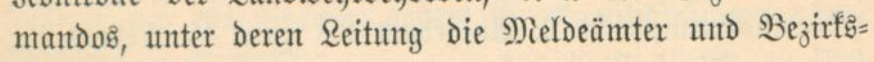
feld webel itehen.

Meldec̈mter beitehen an Drten, wo mefrere Sompagnie= bezirfe ifgren Stationsort Gahent. Die Meldeämter an Dent

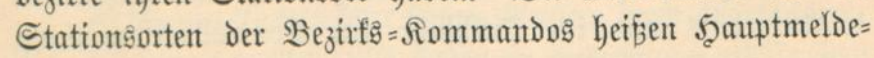
ämter.

Fontrollbezinfe find die Randwehrbejinfe und interhalb derfelbent die Rompagniebejirfe bezm. Die Bezinfe der Melde= ämter.

Smmerhalb 14 Iage nady Der Entlaffiutg und fpäter

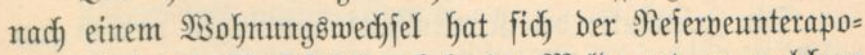
thefer bei dem Bezirfsfeld webel oder Meldeamt, zu weldhem

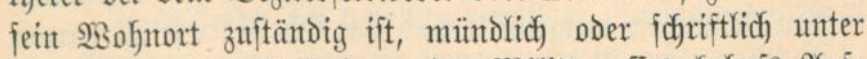

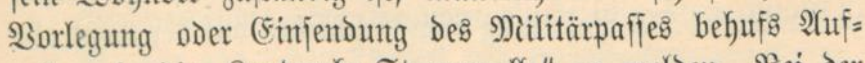

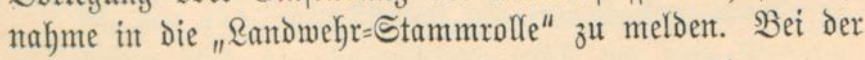


Inthalt

ber

melbung

2kmelbung

vor ber

2roreife

Meloung ift anjugeben: 1) Frïherer Sobnort; 2) jetziger $\mathfrak{G o h n o r t ; ~ 3 ) ~ F a m i l i e n f t a n d ~ ( o b ~ v e r h e i r a t h e t ~ u n d ~ w i e ~ v i e l ~}$

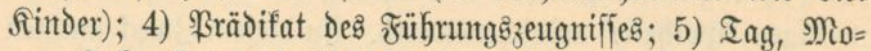
nat, Sahr, Drt und Fräbifat der Îpprobation.

Berändert der Beurlaubte feiuen $\mathfrak{B o h n f i b s , ~ j o ~ m u ́ s ~ e r ~}$

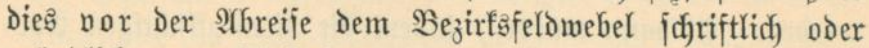

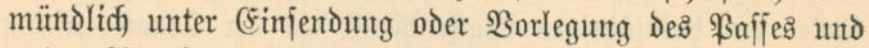

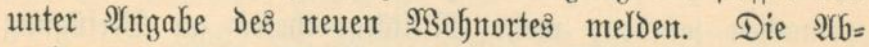
meldung nad) ber N(breije wird, went fie nidjt durd) unvor= hergejebene Erreigniffe veranlä̧t ijt, wegen Sontrollentziebung beitraft.

Dienftsfliat Der Unterapothefer gebört, wie fadjon oben gejagt, der

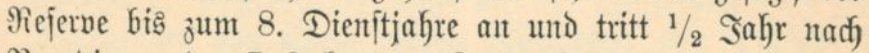
Beendigung Des 7. Jahres zur Randwehr 1. Nufgebots über.

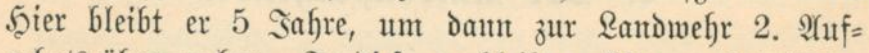

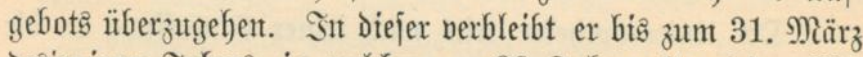
Deติjentigen Jahres, in weldjem er 39 Jahre alt wird. Mit

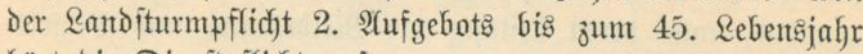
Gört die Dienftpflidjt auf.

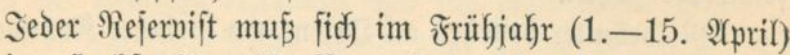

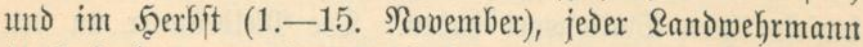

Sontrolle veriamm: lutgent

1. Aufgebots mur im ₹rübjafyr jur Sontrolfverjammlung ftellen; Der Landmebrmant 2. $\mathfrak{2}$ ufgebots ijt frei dawout. Sur $\mathfrak{A} n=$ und $\mathfrak{A}$ bmeldung find aber alle orei filaffen berpflidytet

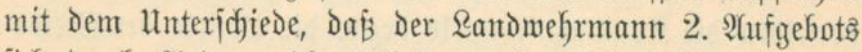
fidf Durd) S(noere, Die Hebrigen aber fid). jelbit ant= und abjumeliden babent.

Die Beitpunfte Der Rontrollnerjammlungen werden nur Durd) 2 (njid)lag an öffentlidgen Sorten uno Befantmad)ung in Den Beitungent mitgetbeilt und bat jid Seder jelbjt barum su fümmern. Die Befreunty vom (Erjd)einten auf Der Rontroll= 
verjammlung muí ungefähr 10 Inge vor Derjelbent beim Bezirfsfeld webel beantragt werden.

Yuf Der Sontrollverjammlung treten Die Itnterapothefer vor Den Mannjdaften in Der Reibe Der Unteroffiziere ant.

Dą Berjäumen der Rontrollverjammlung obne red)t= zeitige (Entjduldigung wird disziplinarijad) beitraft.

Die Dişziplinarverhältnifje find biejelben, wie diejentigen DiąziplinarDer einjührig = freimilligen s) Silitürapothefer, D. $\mathfrak{h}$. Diejentigen

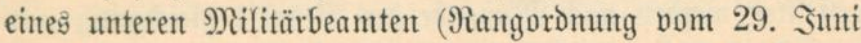

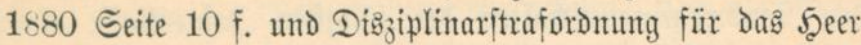
vom 31. Dftober 1872 Abjdyitt IV. Seite 37).

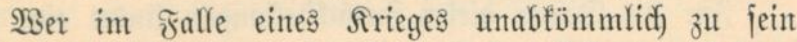
glaubt, fam fid) binter die leb̧te Sahresflafle der Rejerve bezm. Ranbwehr zurïfitellen laffen. Soldye Burïfiftellumgen leste Sabres.

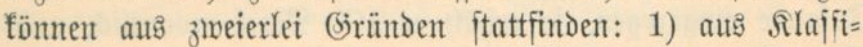

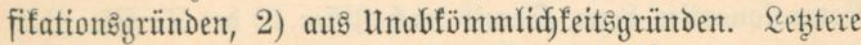

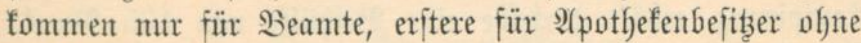
(Siebülfent in Betradt.

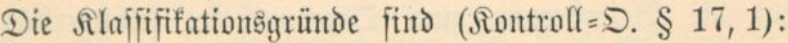

a) menn ein Maum als Der einzige (Enäh)rer jeintes arbeits:

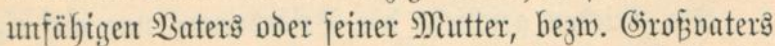
oder (Sirofimutter, mit Denten er diejelbe Feuerftelle be= wohnt, zu betradyten ijt, und ein fined)t oder (siejelle nidjt gefalten werden famm, aud durd) Die der familie bei der (Ëinberufung gejeb̧lid) jujtehende Unterjtüb̧utg

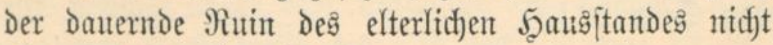
abgemendet werden fömute;

b) พent Die (Finbernfung eintes Miautes, Der Daร 30. Rebents: jahr vollendet hat und (Brundbefitier, Fäd)ter oder (joe= werbtreibender oder (Ernährer einter zablreid)en Familie

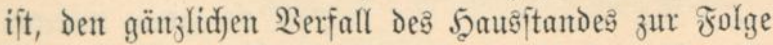
haben und die singehörigen jelbjt bei dem (5jenuffe

Brüntbe für bie 3urūct. ftellumg 
Der gejeb̨lidfen Unterjtübung Dem EYlende preis̈gebent mürde;

c) went in einzelnen Dringentoen Fä̈llen die 3urüfftellung

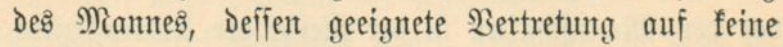
2Beije zut ermöglidjen ift, im Intereffe der allgemeinten

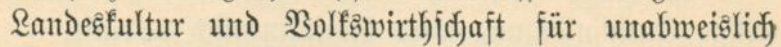
eradjtet miro.

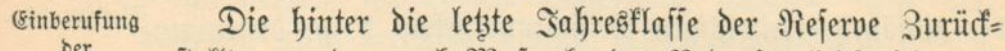
ber Burüct. Beftellten

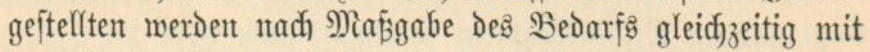
dent Randwefrmantujaften der jüngften Iahresfllaffe eint= berufen.

Für die Dauer diejer 3urüffitellungen findet eite $\mathfrak{B} e=$ förderung nidyt ftatt.

Einfommen im falle ser (Einziebung

2Bird ein Unterapothefer im Siregŝfalle zum Dienit im Intande eingezogent, fo erbält er 100 Marf montatlid) und

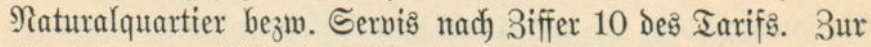
Equipinung erbält derjelbe eine Beibülfe von 105 Mart. Die Berleifumg einer vorjd)riftêmäfigen Feldapotheferjtelle hat die Beförderung zum Dberapotbefer jelbjtwerjtändlid, fur Folge.

Im Friedent gefdieft die Beförderung nur auf eigenent Antrag nad) 2 jähriger Dienjtzeit in Der Rejerve.

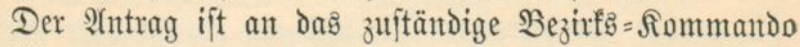
ölt ridftent.

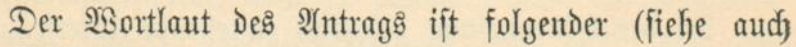
oben Eeite $22 \mathrm{f}$.$) :$

Drt, Den . .tent Monat $18 \ldots$

2tu

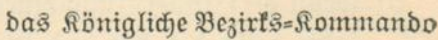

out

Das Röniglidje $B$ ezirf'S = Rom= manos bitte idj geboriamit unter 


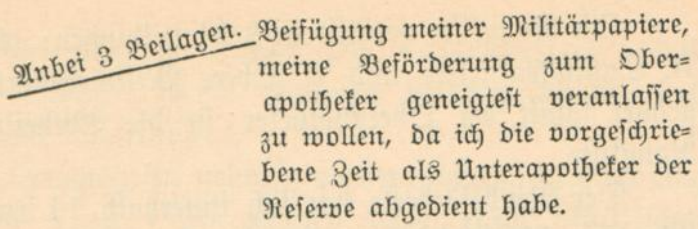

Name.

Unterapothefer Der Rejerve.

Der $\mathfrak{A}$ ntrag ift mit dem Führutgågengnif́, der $\mathfrak{B} e=$ ftallung unto Dem Militärpaffe Dem Bejirfafeld webel zu über= geben, weldjer die 2 Seiterbefürderung bejorgt. Die Beförde $=$ Beförberung

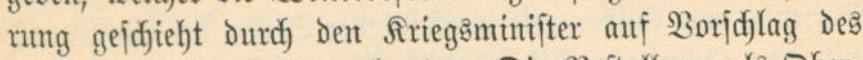

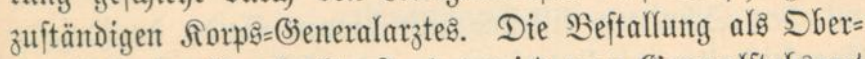
apothefer des Beurlaubtenftandes wird nom Beneralftabsan auşgeftellt. Die Befördenutg wird vom $\mathfrak{B}$ ejirf

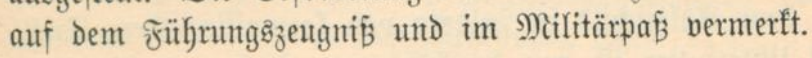

\section{Ber (1) Gerapotyeker.}

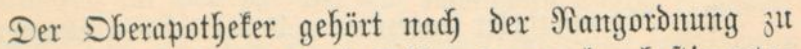
Dent oberen Militärbeamtent mit Dffiziersarantg ohne beftimmtent (brad. Es finden auf ifn alle Beftimmungent, weldhe für Dffizicere des Beurlaubtenftandes erlaffen finto, fintgemäf̌e Inwentoutg.

Heber jedent Difizizer wird ein bejonderer. Ferjontalfogent aufgeftellt, weldyer aufer dem Nationale alle $\mathfrak{N}$ (ngaben iiber bogen

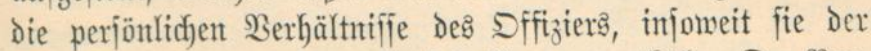
Mitlitärbehörde von Intereffe fein fömtent, enthält. Der $\mathfrak{Q}$ er= fontalbogen wird vom Dffizier felbft ausgefüllt.

WSie der Unterapothefer nom Bezirfśfeldowebel in der Ranglifite Randwefyrtammrolle, jo wird der Dherapothefer nom Bezirfs=

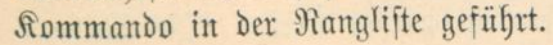


Subalt ber Ranblifte beim:ezirfą

Sromt: manbeut

Uniform

Die Ranglijte jerfällt in 3 श्tbtheilungen: A. Difizziere, B. Sanitätsoffiziziere unt C. Sbere Militärbeamte, und ge= gehört jomit der Dberapothefer in bie Afbtheilung C. Der Ranglifite.

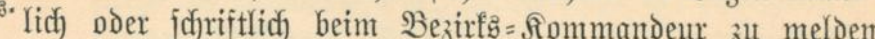
(fiehe oben Seite 27 f.). Die mündlidje Meldung gejdjefyt in Uluiform.

Untiform

Die Uniform unteridjeidet fid, von Derienigen Der eint= jüfrrig=freimilligen Millitärapothefer Dadurd), Dá Der Degen ein \$ortepée von Eillber mit bunfelblauer Seide, der $23 a f f e n r o f f$ auf Dent Ecfjultern filberne mit blater Seide Durdjwirtte Epaulettbalter mit Unterfutter von Dunfelblauem Iud) trägt. Daz̧u fommen Epaulettę mit goldenem, geprejten Firanz

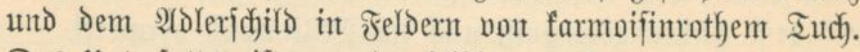
Das Unterfutter ift von dunfelblanem Iutd) unto die (Ein= faffung von goldenter Irefje. Die Epaulettes fümen exjetzt

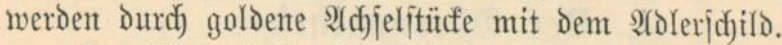

Der Mantel ift ofne Edjulterabzeidyen.

Die Dberapothefer tragen einen Meberrouf von jojwarzent, in Bayern Dunfelblauem, Tudje und Siragent vont Duntel= blautem Iutue, mit farmoifinrothen Borftöfent um den Siragen,

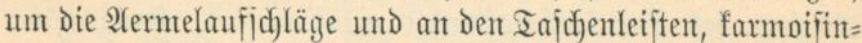
rothem Rlappenfutter mit vergoldeten, in Bayern verfillberten,

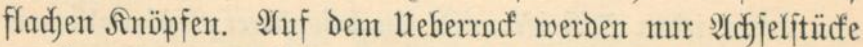
angelegt, Darum fehlent die Epauletthalter. SAud jonjt wer= Den auf Dem 23 affenrocf die Epaulettes mur Dant getragen,

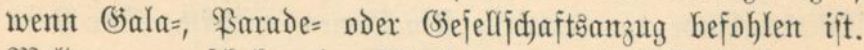

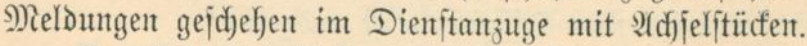

Der Fुelm der Rejerve= Dberapothefer trägt am oberent Theile des Stolers, Derjenige der Randwehr $=$ Dherapothefer am

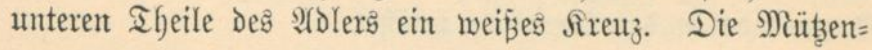


fofarde beider Slaffen enthält das Ranbwefrttrenz. (NB. Freupijidje Beamte, Deren Uniform filberne Sinüpfe hat, haben filberne צdojjelitütfe.)

Die Sberapothefer nehmen an der Sontrollwerjammlung in Uniform Theil. Sie werden Daza Durd. fderiftlidjen Befehl beftellt und ijt Diejer Befehl, nadjoem der Iag der Sontroll= Beriamm: Iung

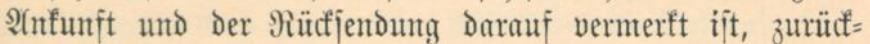
子uj juifen, 子. $\mathfrak{B}$.:

$$
\begin{aligned}
& \text { In Den ..ten Mionat } 18 \ldots
\end{aligned}
$$

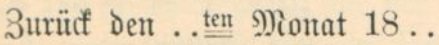

$$
\begin{aligned}
& \text { unterjudrift. }
\end{aligned}
$$

Faf Der Sontrollwerjammlung hat fid der Dberapothefer bei Dem Difizizer, weldjer bie Rontroffverfammlung abhält (Rontrolloffizizer), zu meldent (fiehe oben Seite 27 f.).

Der Mantel Darf mur dam angezogen werden, wemt Der bie $\mathfrak{B}$ erjammlung abhaltende Difizier mit angezogenem Mantel eridjeint. Den Mantel im Dienft umzuhängent, ijt unftatthaft. (Es darf das mur auf bejonderen Befehl erfolgent.

SBer fid) zur Beit der Rontrolfverfammlung aujerfalb

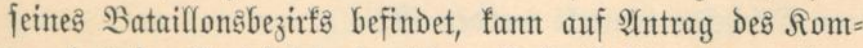

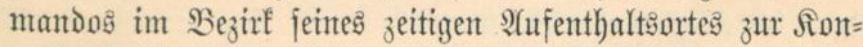
trollverjamlung herangezogent werdent.

פser von der Siontrolfverjammlung befreit feit will, hat

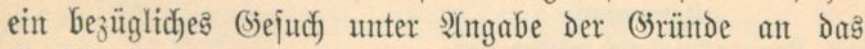

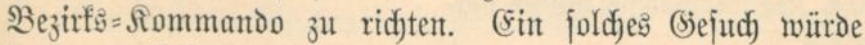
in Der umftehento angegebenten Siseije abzufajjen jein. 
(5eiud) $\mathrm{um}$ Befreiung von ber Sontrollver.

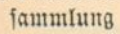

Drt, Den . ten Monat 18 . ..... Str. Nir. . 2trn

Den Röniglidjen Dberiflieutentant

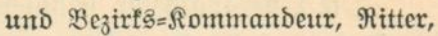
5eerrn .......

5od)wohlgeboren jut

\section{Drt.}

(Euter 5ุod)mohlgeboren bitte id), miti) vout ber Theilnahme an ber am 12. ๖. M. 10 Hhr Bormittag Gier itattfindenden Rontrollver= fanmtung befreien zut wollen, $b a$ idf an biefem Tage eine nothwent= bige (sejdäfts(Dientit)reife ntad) ...... antretent mú

\section{Name.}

Dberapothefer Der Rejerve

(\&anbwelyr).

Diejes (Si)jud fommt mit dem Bejdecide deకి fom=

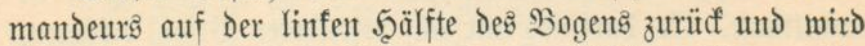
Dant abermals an Den fiommandeur gejojifft, nadjoem unter Dem Bejdeide bemerft worden ift:

$2 \mathfrak{n}$ Den ..tent Mionat 18 .

zurüct Den . ten Monat 18 ..

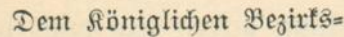

Rommanoo

nad) Rentntifnabme wieber vor=

gelegt.

\section{Rame.}

Dberapothefer Der Reierve

(Zanbwelyr).

Die Befreinty ift jo jeitig zu beantragen, daj der be=

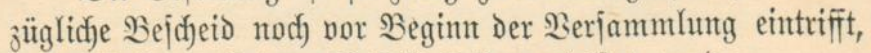
andernfalls muí dem $\mathfrak{B} e f e \mathfrak{h l}$ Folge geleiftet werdent. 


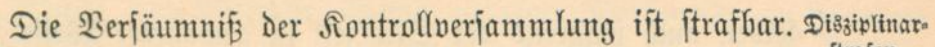
Die Sherapothefer find als obere Militärbeamte derielbent ftrafent Straforomung untermorfen, wie bie einjäfrig $=$ freimilligent Militärapothefer (fiehe Seite 37). In diejer Dişziplinaritraf=

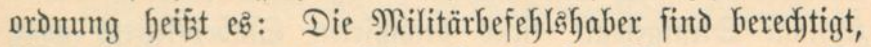

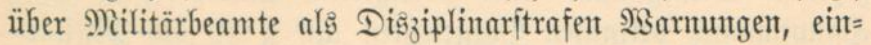
fadje Bermeije und gegent obere Militärbeamte (jeldbutie bis

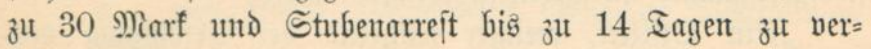
häntgent.

Die hödjte Beldbufie darf nur der fommandirende (je $=$ neral, die übrigett Borgejeł̧tent mur biš zum Betrage vout 9 Marf verbättgen.

Bur Berbängung von Bieldiftrafent finto unr Diejenigent befugt, weldje Dffiziere mit Stubentarrejt vout beftimmter Dauter beftrafen dürfert. (Die Dffiziere fömten Etubenarreft

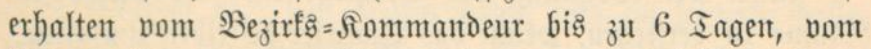

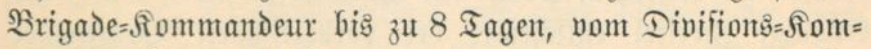
mandear bis zut 10 Ingen und vom fonmandirenden (bje= neral bis ż 14 Iagent.)

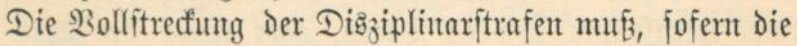

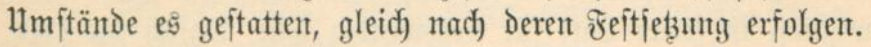

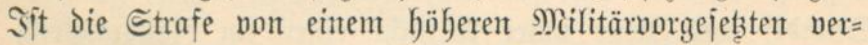
bängt, fo bleibt es jeinem Ermtefien überlafjen, die $\mathfrak{B o l l}=$ ftreffutg derfelben entweder jelbjt anjutordnen oder dem

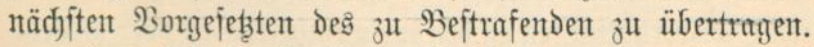

Der einfache Stubenarreft wird vont Dem Berurtheilten

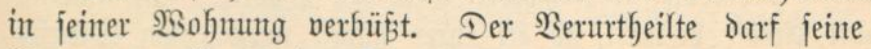

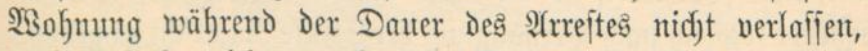
audd) Befudde nidjt autefymert. Dem $\mathfrak{A r}_{3}$ te ift der 3utritt jeder Beit geitattet.

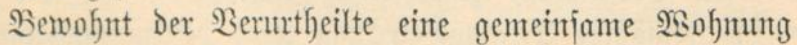
mit moeren Ferjonten, jo faut er Den Berfegr mit Dent= 
jelben aud währento Der Strafjeit in gewohnter æietje fort= jeţent.

Seder zu Stubentarrejt verurtbeilte Difijier ijt bei dem Strafantritt Durd) den nädjiten, mit mindejtents Der Diછ̌= ziplinar = Strafgenalt eintes detadjirten Stabsoffiziers oder

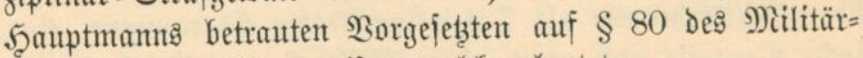
ftrafgejełzbudjes Ginzuneijen, weldyer lautet:

"Ëin Dffizier, weldyer mährent Der Berbü̈ung Deڤ

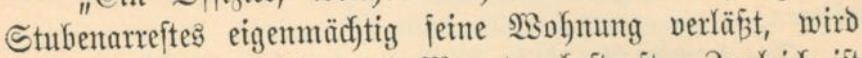
mit Freibeitsftrafe bis zu 6 Mionaten beftraft. Sugleid) ift auf Dientitentlaffung zu erfenten. Ëin Dffijier, weldjer

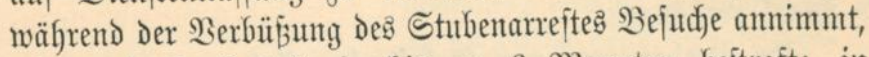
wird mit Freiheitştrafe bis zul 6 Mconaten bejtraft; in jojweren Fällen ijt zugleid) auf Dienjtentlaffung zu erfenten."

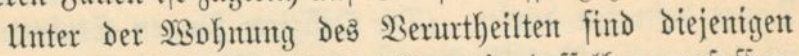

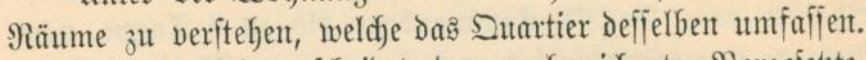
Etwaige 3weifel entjdjeidet Der norbezeidjutete Borgejęte. Setsterer ift aud befugt, einjelnen \$erjonen in oringendent

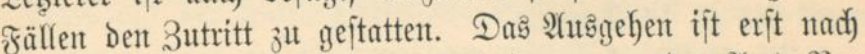
einer Strafbauter von 14 Iagen, oder went Der $\mathfrak{A}$ rajt Be= wegung in freier $R$ afft für nothwendig bält, täglid) für eine Stunde, und zwar unter Suffidyt einer im Rantge gleidy) oder höher ftehenten Mititürperjon zu geitatten.

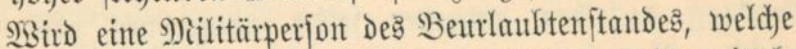
in ifgren (Sivilberbältnifijen zu den im unmittelbaren oder mittel= baren Staatšdienjte ftehenden Beamten gehört, disziplinarija nit $\mathfrak{A}$ rrệt bejtraft, jo ijt ibrer nädjit vorgejetżen Dienjt=

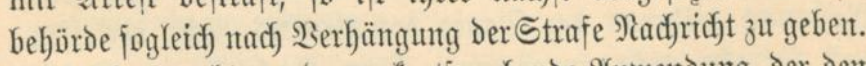

Die gered)te und zwedfentipred)ente Anwentung Der Dent niederen Sorgejetzten zuitehenden Strafbefugnifie und bie

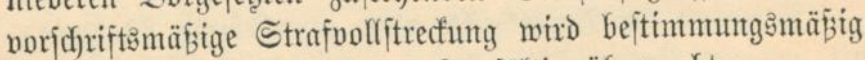
von den höheren Borgeję̧ten jorgf̈̈ltig überwadyt. 
(3)lanbt der Beftrafte (Grumb zu einer Bejajuerde ju Beidwerbe-

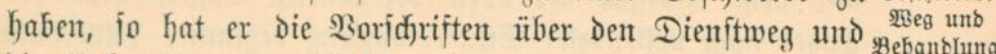

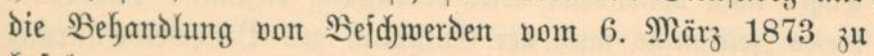
befolgent. beadfent:

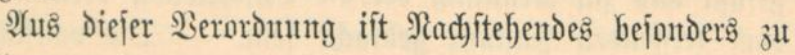

Die Bejalwerde fant zum Biegenjtande haben:

1) eine von einem $\mathfrak{B}$ orgejetzten verfängte Dişjiplinar= itrafe,

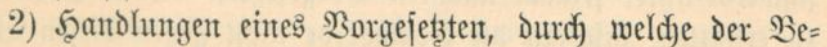
jd)merdefübrer perjönlidf) oder in jeintem beredftigtent

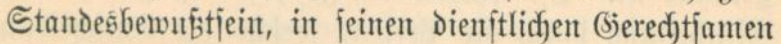
unt Befugniffen verlebzt wird.

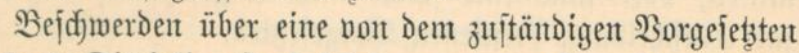
verbängte Diszziplinarftrafe Dürfen mur von einem Borgejebsten Des Bejtraften oder von diejent jelbjt, und im leb̧teren Falle erit nad) der Strafuollitrecfung und ohne Mitwirfung eines

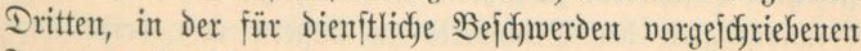
Form angebradjt werden.

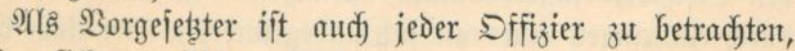
weldjer fid berpflidytet fühlt, gegen einen jüngeren fiame= radent dienfitlid einzuld jreitert.

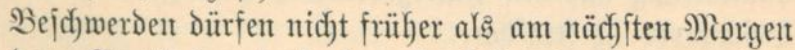
nad) Dem Stattfintoen Dešjentigen $\mathfrak{B o r f a l l a ~ e i n g e l e i t e t ~ w e r d e n t , ~}$ weldjer zur Bejdjwerde Beranlaffung gegeben hat. Eine Suร̃nabme von diejer Borjodyrift ijt zuläffig, ment durd) Imtebaltung Derjelben bie Entijdeiduntg mejentlid) erjodwert wird, ober eine 2erjögerutty erleiden wïrde, weldje in $\mathfrak{B e}=$ rüffidjtigung des Spejialfalles bedenflidy erjocint. - Bor Beendigung des Dienjtes darf Der Bejdfwerdemeg niemal: betreten merdent. Bur YAtbringung Der Bejdjwerde miro dem Bejdjwerdefübrer eine Frift von orei Iagen gegeben, weldye 
mit Dem Morgen Dew oben bezeidunteten Tages, oder Deڤ Iages nad, volfendeter Strafuerbüfung begimnt. Smmerhalb

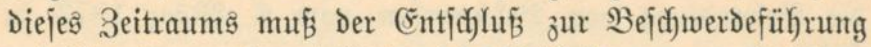
gefabt und zur Senntnifi der die Enjd)eidung vermittelnden oder treffenden Injtantz gebrad)t jeint. - Die vollftäntige 5erbeija)affung Des Materials und Die etwa erforderlidye Be=

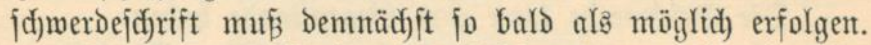

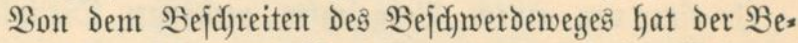

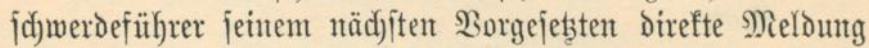
zll erftatten, injofern die Bejd)werde nidjt gegen diejen jelbjt geridftet ift. Sit letzteres der Fall, fo erfolgt die betreffende Mittheilung Durdid Die Ferjon, weld)e die (Entjdeidung ver= mittelt, oder falls einte joldfe nidjt eingejestst ijt, Dutrd) dent zur Enticheidung berufenen Dorgejeţtent.

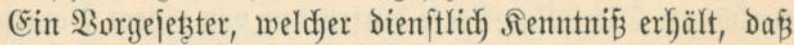
ein ifm untergebenter Dffizier beabjidjtige, über einen $\mathfrak{B}_{\mathrm{or}}$ gejebzten Befdymerde zu führen, ift bered)tigt, fid) über etmaige (Sirumblofigfeit der Bejodyerde zu äu[iern und nerpflidjtet, Den $\mathfrak{B} e j d$ jwerdefüfrer darauf aufmerfjam zu madjent, bafi er Durdd Anbringent einer an fidc) unbegrïndeten oder einer leid)t= fertigen Befolywerde fidf ftrafbar madje. Cine andermeitige Eimwirfung auf Den Untergebenen bebufs 3urüfjiefung der Bejolumerde ift ftrafbar.

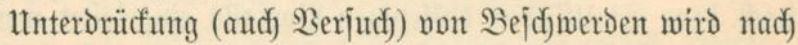

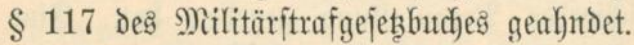

In exjter Inftutz entjd)eidet über eine Bejodwerde in

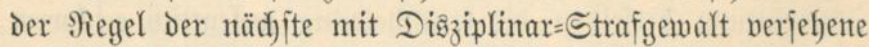

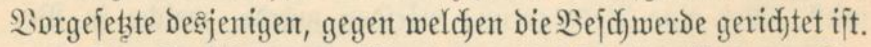

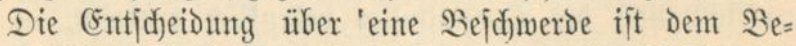
jodwerdeführer, jowie dem hödjiten der non der Bejadyerde

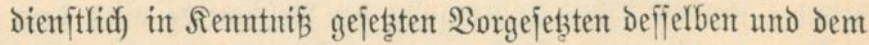
Sertlagten mitzutheilen. 
Biegen bie getrofiene Entidfeidung faun von beident Theilen, innerbalb 3 Iagen nad) Mittheilutug Derfelben, $\mathfrak{B}=$ rufung an die nädfit höbere $\mathfrak{S} n \tan _{3}$, und fo fort, ohne $\mathfrak{u m}=$ gehung einer Snftanj bis zur allerbödjiten Stelle hinauf erboben werden. Die Berufung mird in Bjeitalt einer $\mathfrak{B}=$

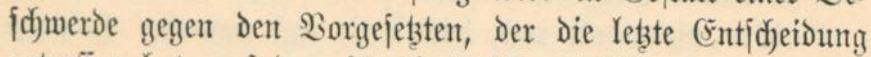
getroffen hat, auf Dem für Die Bejdymerdeführumg geordnetent

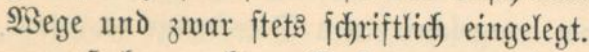

5ुabent mefrere Ferjonen aus gleidjer $\mathfrak{B}$ erantlajfung $\mathfrak{B} e=$ fajwerde zu führent, fo ift bie Ânbringung der gemeinfament

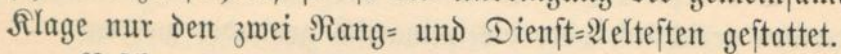

Bejdjwerden, weldje von Seiten des entifjetidenden $\mathfrak{B}_{0}=$ gejestent für unbegrüntdet erad)tet merden, weil fie von faljoden

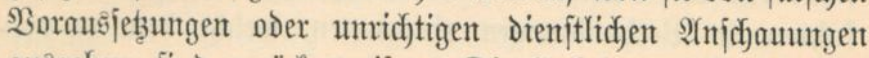

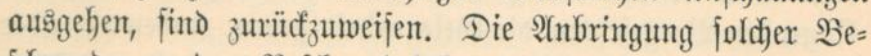
¡djwerden an Dem Bejdywerdeführenden zu ahnden, bleibt dem Ermefient des enticheidentoent Borgejetztent und, falls diejer zur

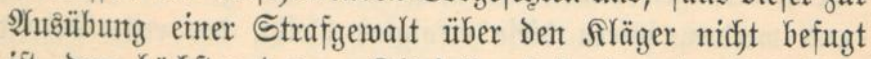
ît, Dem hödjften des zur Dişziplinarbeftrafung des BetreffenDen fompetenten, von der Sadje dienftlid) in Remutnifi ge=

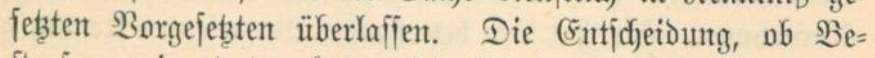
ftrafung einjutretert bat, miro fid) Darnad) ridjten, ob die Aufredterffaltung Der Buddyt umb Dromung im Şeere eint Derartiges (Einfld)reiten gegent Den Fläger erfordert und Die Eage Der gejetzlidyen $\mathfrak{B}$ eitimmuntgen ein Strafperfahren an=

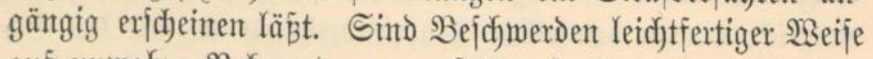
auf unwabre Befauptumgen geftiibzt, fo liegt eine, erforder=

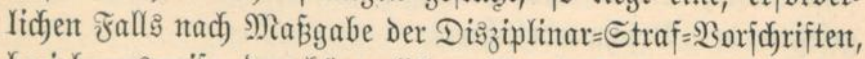
bezichungsmweije Der bürgerlidjent Strafgeję̧e zut ahndendent

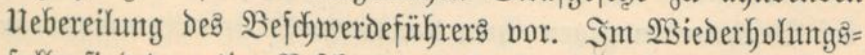
falle fint derartige Bejdymerden ebento, wie die wider Gefferes 
Biffen auf unmahre Behauptungen geitübtent an Berjonen ঠeริ Soldatenjtandes gerid)tlid) zu ahnden.

$\mathfrak{B}$ er wider befferes $\mathfrak{B}$ iffen eine auf unwahre $\mathfrak{B e b a u p =}$

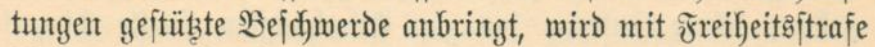
bis zul einem Sahre bejtraft.

Wer wiederbolt und Yeidjtfertig auf ummabre Bebaup= tungen gejtützte Bejdjwerdent oder wer pite Bejadyerde unter Afbeidung von Dem vorgejdriebenten Dienftwege einbringt,

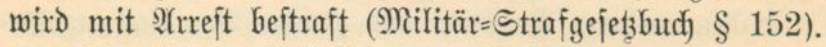

Der leb̧tere Fall fant an Berjonen des Beurlaubtent= ftandes geridytlid) oder disziplinarijd) geabndet werdent.

Dffiziere fint verpflidftet, bewor fie ifre etwaigen $\mathfrak{B} e=$ jdjwerden Der Entidjeidung Des fompetenten $\mathfrak{B o r g e j e t z t e n ~ z u t = ~}$ fïfren, in Berbandluntgen einzutretent, weldje dem zit ver= flagenden Borgeję̧tent die Sielegentyeit geben, unbemufit oder in Uebereilung zugefïgte 1 turedyt jofort abjuftellen. Dieje Berbandlungen bilden den 2 Beg Der dienjtlidjen Bermittelung und werdent, jofern dieje exfolglos bleibt, Der exite Sdyritt

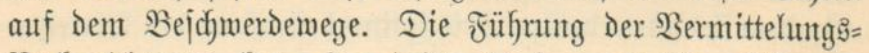
Berbandlungen hat eite Dritte $\mathfrak{F e r j o n}$ als $\mathfrak{B}$ ermittler zu übernefment. Sie hat Dem betreffenten Borgejetzten Rentntnif

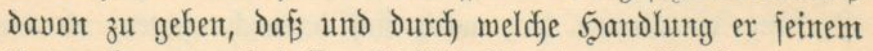

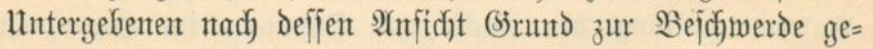

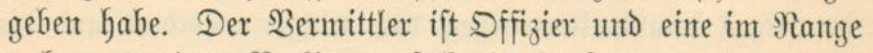
nahe unter Dem Berflagten ftehende Serjon, und jomeit als möglidy, ein zu demjelben Iruppenwerbante 2 . wie der $\mathfrak{B} e=$ jofwerdefüfrer und der Berflagte geförender direfter $20 r=$

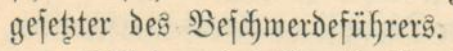

Dffiziere des Beurlaubtenftandes Gaben als Bermittler

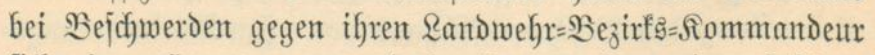
fidf) eintent 5̧auptmant, unt in Ermangelung eintes joldjent, einten älteren Dffizier deร Beurlaubtenjtandes aนร írem 
Bataillongి = Bezirfe zu wählen. Der zum :ermittler Gie= wäblte darf die llebernafyme mur Damn ablebnen, went er entweder die Bejd)werde in allen $\mathfrak{B u n f t e n}$ für vollfommen

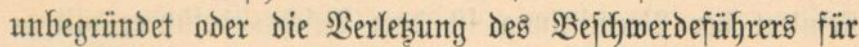
eine jo jajwere hält, dẩ er eine Bejeitigung Derjelbent im ISege der $\mathfrak{B}$ ermittelung nidyt für thunlid, eradjtet. Nebent

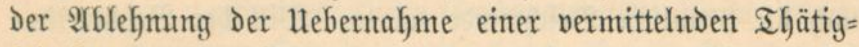
feit hat Der Betreffende im erfteren æalle von Der (sintreid)ung Der Befd)merde abzurathen, im leţteren Dem Âläger die Direfte Eingabe Der Bejadjwerde ankeimzuftellen.

Die Ibätigfeit Des Bermittlers beginnt damit, dấ er fid) Durd, Den Bejdymerdefülyrer genau über die einzelnen Bejumerdepunfte unterridyten läbt. $\quad$ Er ift beredftigt, die jdriftlidje গiederlegung diejer Funfte und des diejelben be= grïndenden Ifatbeftandeg zu fordern, und aud berpflidytet,

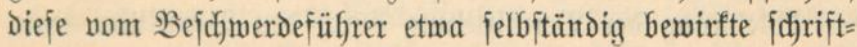
lidje formulinung der $\mathfrak{B}$ ejdywerde anzutehmen. Der $\mathfrak{B}$ er= mittler hat jelbft zu ermeffen, ob er Dem Berflagten die fdoriftlidje Darlegung zur Renntnifi vorlegen Darf, ofne den

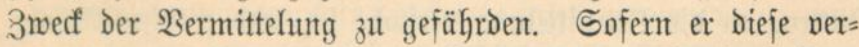
neinen muß, ift Der Einbliaf in Die Rlagejdyrift Dem $\mathfrak{B e r}=$ flagten vorzuenthalten. Âtud hat der $\mathfrak{B e r m i t t l e r}$ die $\mathfrak{B} e=$ fugnifi, Dem Bejdywerdeführer jeine $\mathfrak{Y} n$ fidjt über nidjt ge= nügento Begründung der fllage funt zu gebert. WSird bie (Eintleitung einer foldjen, burd) den $\mathfrak{B e r m i t t l e r ~ a l s ~ n i d d t ~ g e = ~}$ nïgento begründet bezeidneten Bejdywerde bentod) vom $\mathfrak{B e}=$ jd)merdefithrer begehrt, fo bringt der Sermittler bas durd) Die Berbandlungent gemonnente Material momöglid. mündlich zur Renutnifí des Berflagten, jpridft demjelben auf Befragen

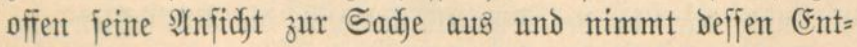
jdeidung Darïber entgegent, ob Derjelbe beabfidftigt, die $\mathfrak{B}$ er= anlaffung zur Bejumerde aufatbeben, oder diejelbe Dem 
fompetenten Borgejeţten ふur meiteren Bejđ)lubfafïung zu=

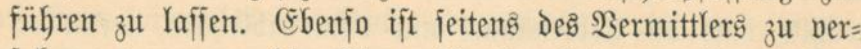
fahren, went er die Sllage für begründet erad)tet. Da Rejultat der Bermittlung ijt Dem Bejdymerdefïhrer mitzu= theilen.

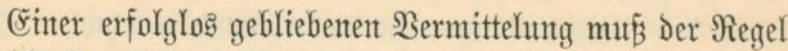

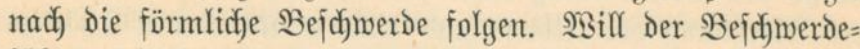
füfrer jebod), bemogen durd, die im Raufe der Berband= lungen gemonnente Eimfidft, jeine Allage zurïffieben, fo ijt dies jtatthaft, jofern nidjt Derjentige $\mathfrak{B}$ orgejebste, gegen meldjent

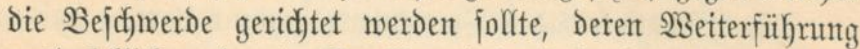

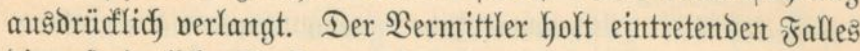

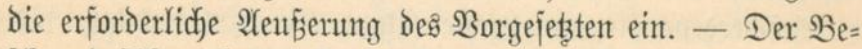

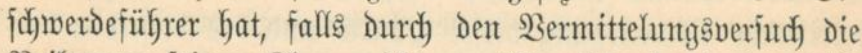
Beilegung jeiner Silage nidyt erreidyt wird, jowie im falle

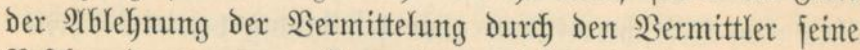
Bejofjwerde, went er fie weiter verfolgt, bei bem zur (Ent= jojeibung Derjelben fompetenten Borgejetzten münolidy oder ¡d d)riftlich jelbjt vorjutragen und gleidjzeitig Meldung zu er=

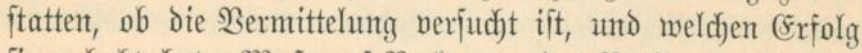

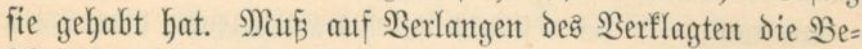
(d) fwerde nad exfolglojer Bermitteling weiter gefïlytt werdent, jo ïbernimmt Der $\mathfrak{B e r m i t t l e r ~ D e n ~} \mathfrak{B}$ ortrag. In Denjentigent

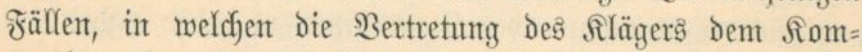
mandeur des Iruppentheil's 2 . obliegt, hat diejer die $\mathfrak{B} e=$ jojwerde bei dem enticheidenden Borgejeşten antzubringent, weldyem es in allen vorgedadyten Fällen ïberlafjen bleibt,

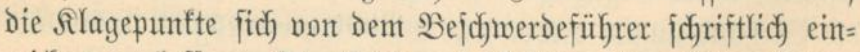
reidjen zu lafjent. Die Bejdywerdejdyrift mub rubig gebalten

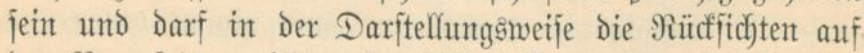

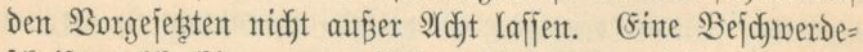

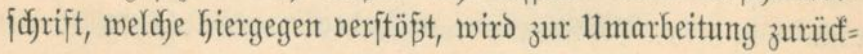




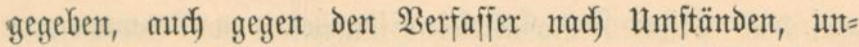

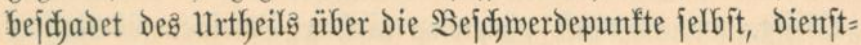
lid) eingejodritten.

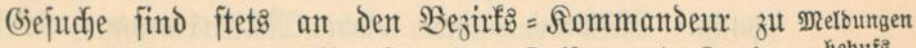

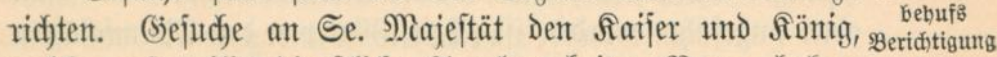

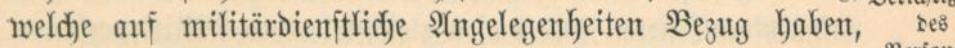

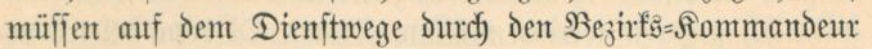
vorgelegt werdent. Ŝlnderenfalls geben fie unbeantwortet ant den fommandirenden (sieneral jurïif und der (siejud fiteller wird mit $\mathfrak{\text { frrejt }}$ bejtraft.

Yuber Den oben gentannten Meldungen bejteht für Dent Sberapothefer die Berpflidjtung, Dem Bezinf: = אommando

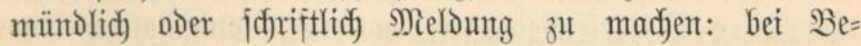
förDerung, bei $\mathfrak{B}$ eründeruntg in Der Berufg̈ftellunty und Den fonjtigen perjönlidben $\mathfrak{B e r b a ̈ l t n i f j e n , ~ j o m e i t ~ j o l d j e ~ i m ~} \mathfrak{B e r}=$ jonalbogen SAfnabme finden müjןen; dahjun gebören z. $\mathfrak{B}$.

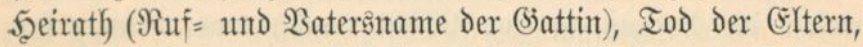

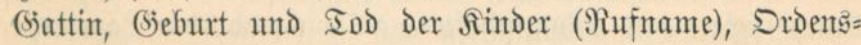
verleihututg.

Neben Den gejeblidy vorgejdyriebenen Sontrollberjamm=

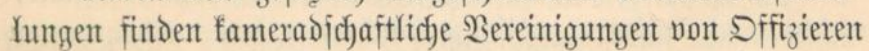
unt Dffiziergbeamten ftatt, gemöbnlidy in Form eines ge= einigungen meinjamen Miables, des jogentanten Riebesmables, weldye in Uniform, die nom $\mathfrak{B} e$ jurf: $=$ Siommando beftimmt wird, $\mathfrak{a} \mathfrak{b}=$ gehalten werden.

Die bayrijden und mürttembergijaden Dberapothefer werden in Den bezüglichen $\Re a n g=$ mnd Duartier=Rijten gefübrt, die prenfijd)en aber nidjt, obmohl andere obere Militär= \$erional= bogent

Samera idaftlidie Ber= beamte Darin zu finden find. SBarum find die prentijd)en Sberapothefer nicht ebenjo müroig, wie bie banrijdyent und württembergijd)en fiameraden, wie Bablmeifter, Sberrofärazte

Dffiziter= Rang= unto Quartier: sitite 
u. . $m$.? Reider fint Rolleftiv=Eingaben von Beamten un=

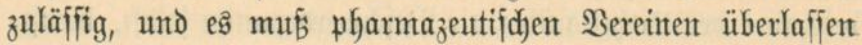
bleiben, darin $\mathfrak{B a n d e l}$ zu jadjaffert.

Nod) anderweitig erleiden bie Sberapothefer eine 3urïf= jeb̧ung. Währent andere obere Mitlitärbeamte, wie jodont oben angefüfyrt worden ift (Seite 36), von Den Mamnjd)aften und Unteroffizierent und biejen gleid)fitehenten Militärperjonent foldatijach gegrüft werden müffen, fönten Dberapothefer von Riemandem ingend meldyen (Sinti beamprudjent. Sie ftehent in diejer Beziebung aujer anderen den Bablmeiftern, Den

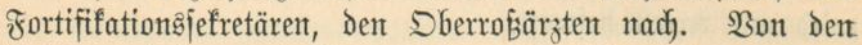

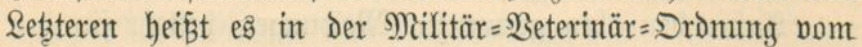
6. )iai $1886, \S 6,2$ : Die Rorps= und Dberrofärate find als obere Militärbeamte von den Mannjdaften und Unteroffizieren Durdy Inleguntg Der Şand an bie Sopf $=$ bedecfung ju grüfent. Dbere Militärbeamte find bie Dberapothefer autd), aber eş fehlt an einer Beftimmuntg,

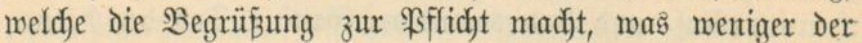
Unteroffiziere und Manmidjaften, als vielmebr ber $\mathfrak{L} a z a r e t b=$ gehülfen megen münjodenţwerth ift.

Beurlaut. bung unt

2นเะ

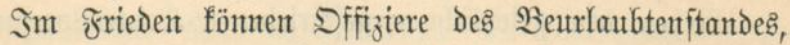
meldye nad) aujereuropäijdyen Ländern geben wollen, unter wanberung Dispenjation von den gemöhnlidjen Dienjtobliegenteitent,

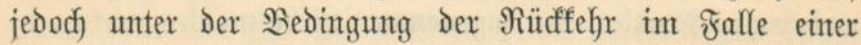

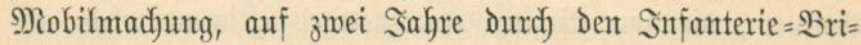
gade= Fommandeur beurlaubt werden.

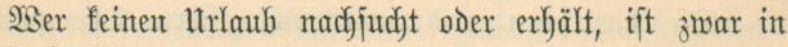

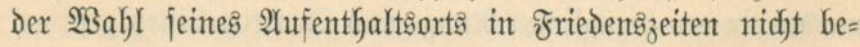

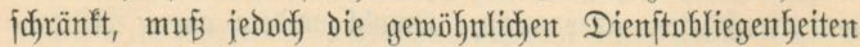
exfülllent.

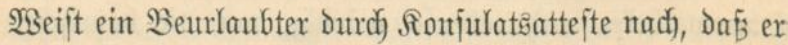
fid) in einem aubereuropäijdyen Rande eine fefte Stellung als. 
Saufmann, Siemerbetreibenter x. ermorben hat, jo fant der

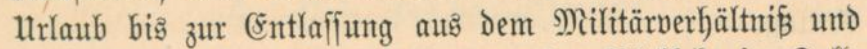

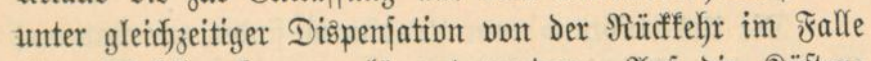

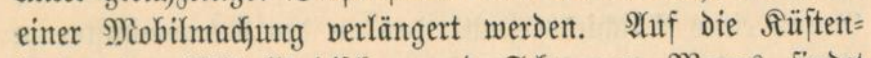

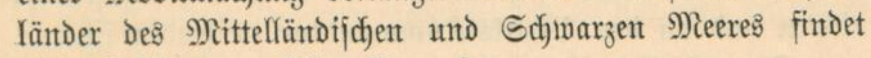
dieje Beftimmung feine $\mathfrak{A}$ (nmendung.

Bei Dffizieren und im Dffizierrange ftehenden $\mathfrak{A}$ erztent ift die Berabjodiesung nadjzujudjen.

Den Dffizieren Des Beurlaubtenjtandes darf, falls fie nid)t nadjmeijen, dấ fie in eintem anderen Butbeştaate die Staatsangehörigfeit ermorben haben - die Ẽntlaffung auร Der Staatåangehörigfeit mur mit (Šntehmigung Der Nilitär= behörde ertheilt merdent.

Derartige Ssejuche find an Das zuftändige Bejirf: = Som = manto zu ridjten.

Dffiziere Des Beurlaubtenitandes, meldje obne Errlaubnif́ aนตืmandern, merden mit Sselditrafe bis zu 3000 Narf oder

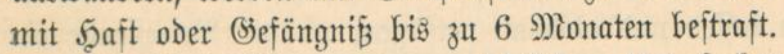

Die Şerbeifülyrung Der geridjtlidyent unterjududung ift

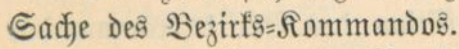

Wenn Berjonen des Beurlaubtenftandes, meldye die Ers=

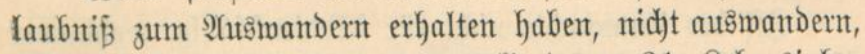
oder went ausgemanderte vor vollentotem 31. Rebentsajare mieder zurüffehren, fo ijt durch die Folizeibehörde dem

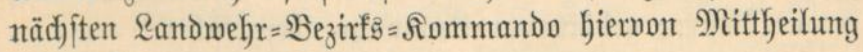
3H madjent.

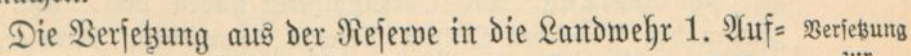
gebots erfolgt Durd) die Bezirf's= Fommandos in derjelben Weije, mie Diejenige der Mannjhaften, ment der Betreffende

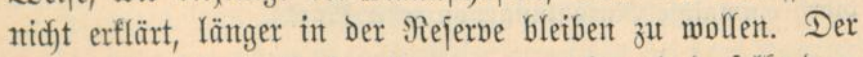
Uebertritt wird im \$erjonalbogen vermerft und Derjelbe dem Betrefienden zum Antrerfenntuifi vorgelegt. Der Hebergang 


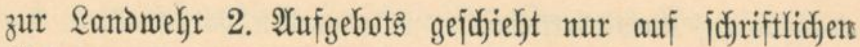
Antrag.

Irbidiet

Die Ueberfübrung jum Randfurm findet mur ftatt auf

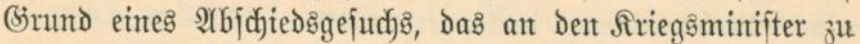
ridjten und an Den Bezirfis=fommandeur zut jenden ift.

Der $\mathfrak{A}$ bjojied ijt in folgenten Fällen möglidu):

1) Dhne $\mathfrak{Z}$ illen des Betreffentent auf $\mathfrak{A}$ ntrag ber vor= gejeţten Ђeeereăbehörden zu jeder 3eit, jowohl vor wie nach) zuriidgelegter Dienftpflidft, ment heeregbientitlidje (Siründe die Berabjojiedung erfordern.

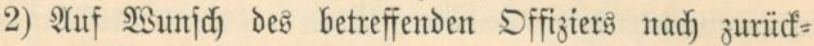
gelegter Dienftpflidy)t oder vor zurïffgelegter Dienft= pflidyt wegen Dienftunbrauds)barfeit.

(S)laubt ein Dffizier dienftunbraud)bar zu jein, jo meldet

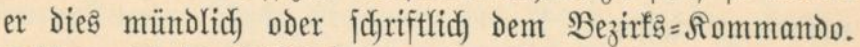

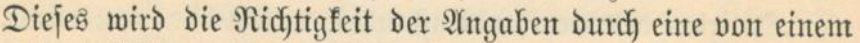

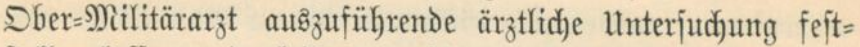
ftellen lafjent und alsbant verfügen, ob der Betreffente ein

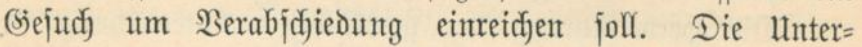

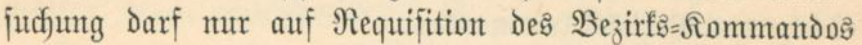
erfolgent.

Sit Der Unterjudofyte nidjt für feldbienitfähig, wohl aber für garnijondientefähig erflärt worden, fo wirb er im Siriegg= falle mur bei immobilen Iruppentheilen verwendet und, fallz er zur Rejerne gehört, zur Randwehr übergeführt.

Anmerfung. Die Dberapothefer haben bas Redjt, Mit= glieber bes Deutfichen Dffizizier = Bereins zut werben. Man erwirbt Die Mitgliebfd)aft Durd) ein 2 (ujuafyme=(Gejud), Das man an Das Direftorium Des Deutidjen Dffizizier=\$ereins in Berlin ridftet und Durch) eimmalige Einzahlung von $3 \mathrm{ebn}$ Marf. 


\section{Ter freldapothelier.}

Der frriegşbienft Der Şeeresanpotbefer wird burd) die

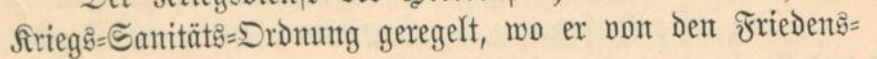
Siriegs: Sanität dienit abweidyt.

Sdjon oben ijt gejagt, daf́ ein Unterapotbefer, ber eine

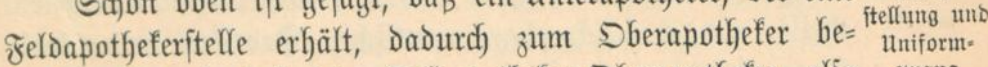
fördert wird, jo dá jeder Feldapothefer Dberapotbefer, arjo smans oberer Militärbeamter ijt. (Er wird burd, die Sorpgigenteral= ärz̧te einberufen und mú uniform tragent.

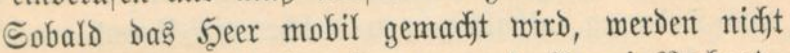

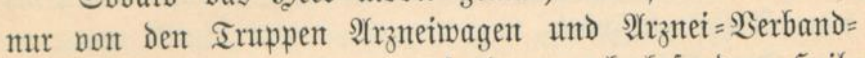

Siriegs: Eanität:̧⺀. Ein. mittel = Siäjten mitgenommen, jontoen aud ) bejondere Şeil= riatumgen unt Bflegeanftalten aufgeitellt, weldye im Frieden idjon vor= bereitet find und in Den Irain = Depots aufbewabrt merdent.

Die \$flegeanjtalten, bei weldyen Feldapotbefer angeftellt werden, finto:

a) Daริ Sanitätsิ= Detadjement;

b) ১aร ซ̌ldlazareth:

c) Daร Siriegălazareth;

Daju fommen:

๖aร $\Re$ Rejerve $=\Omega a_{j}$ areth und

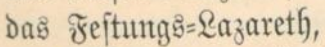

weldye Bezeidynungen mährento Des̊ Sirieges̃ die in offenen StäDten, bezüglidu Feftungen befindlidjen Friedenslazarethe und die in Folge des Sirieges neu eingeridjteten firanfen= anjtalten im Inlande fübren. 2 son Den gentanten $\mathscr{N}$ injaltent fint Daร Sanitätę=Detadjement und daร Feldlazareth bemeg= (ich) und folgen Den Bewegungen der fïmpfenden.

Fenter werden Feldapothefer angeftellt bei Dem Razareth= Rejerve=Depot in Freindesland und Dem Siüter= Depot für $\mathbb{R} \mathfrak{a}=$ 
Die erite

Beriorgung mit 5eil-uns Berbanto. mitteln

Eriał ber berbrauditen Dittel ourd) Requifition

Begriff unb Form ser

Requifition

zarethbedürfniffe ant der Sammelftation im Snllande. Diefe Eintridytuntgen find daza beftimmt, die für die Pfflege der ITranten verbraudyten Sheil=, Berband = unt andere Mittel jut erjetzen, fomeit dies nidjt an Drt und Stelle möglidy ift.

Die erite $\mathfrak{B}$ erjorgutg der Sanitäts̄anjtalten mit $\mathfrak{A r}_{2}$ nte $=$ unto Berbandomitteln gejdjieht theil's aus dent Difpenfitanitalten der Friedentslazarethe, theils aus den bürgerlidyen $2($ pothefen,

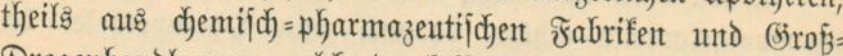

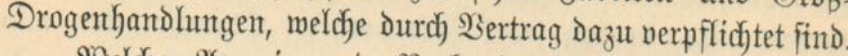

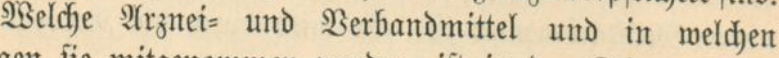
Mentgen fie mitgentommen werden, ift in Der Itriegs: Sant= täts $=$ Dromuntg enthaltent, won weldyer eit (Exemplar fid bei jeder $\mathfrak{A}$ niftalt befindet.

Der Erfałs Der verbrautden $\mathfrak{A}$ rătei $=$ unt Berbant $=$ mittel gejdjieft in Feindeslanto in erfter Rinie durdy $\Re e=$ quifition.

Unter Requifition verfteht man bie went nöthig ge= waltjame Entnabme von in perjönllichem oder behörolidjem Befiţ Gefindlidgen Giegenftände, weldde für die Bedürfniffe des Şeeres nöthig finto. Der Requirirende giebt dem Şer= gebentoen eine Bejdeiniguntg über die $\mathfrak{A}$ (rt und Menge des

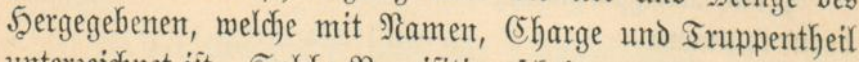

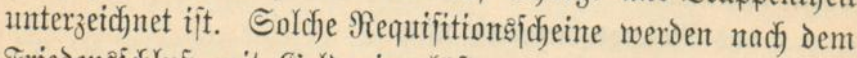

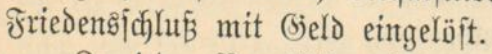

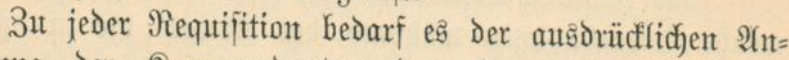
weijung der Rommandantur des betreffenden Etappen= bereidys.

Etabsen, Etappen nent mant foldye Drte in Feindeslant im

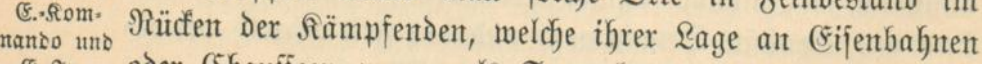
E. Fn: oder Shauffeen wegent als Sammlungs= und Sertheilung punfte dienten für die dent fechtendent Iruppen non Beit ju

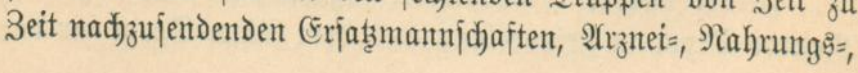




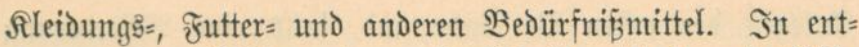

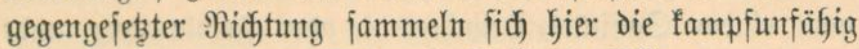
(S)emordenen, um an Dit und Stelle in Siflege gentomment

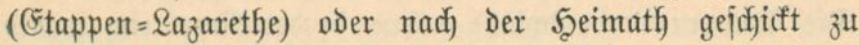
werden. Die Etappenorte erhaltent einte Befaţutg mit einem Rommandantent, weld)er einer Etappen= Snipeftion unterftellt ift. Bei Der (Etappen=Injpeftion befindet fid) ein (Etappen= Sntendant und ein Ëtappen $=$ Sieneralarat mit einem Feld $=$ apothefer.

Die Feldapothefer, weldhe im Ieţten STriege Geritten Bef̈rberung waren, werden jeţ̧ mit \$erjonenmagen gefahren. Diejelfent ftehen unter Dem Sdyubze Der (Senfer Ronvention und Dürfen apothefers eine weife Binde mit rothem Siteuz um Den linfen Sber= arm tragent.

Heber Den $\mathfrak{B}$ erbraud d) der $\mathfrak{A}$ rznei= und Berbandomittel Rednumg: werden in Derfelben $\mathfrak{S B}_{\text {eife, }}$ wie in Den Friedenslazarethen Iegutts

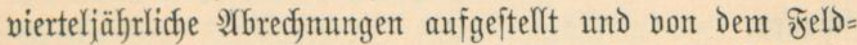
ftabs̊apothefer geprifft. Die ซrormulare werden vom $\mathfrak{L a z}_{\mathfrak{z}}$ areth)= Rejerve=Depot bezogen.

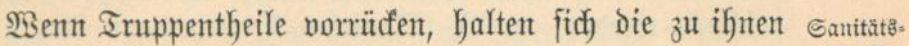

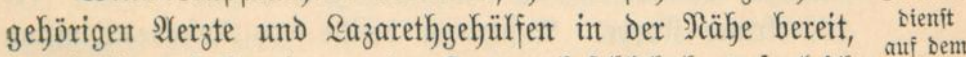
damit fie auf $\mathfrak{A}$ nordmung des Inuppenbefeh) Truppen=Berbantopläbze eintridyten föment. Ferner tretent bie

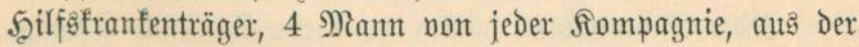
Front, legen eine rothe Binde um Den linfen Dberarm unt

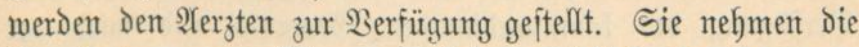
firanfentragent vom 2 Sagent unt folgen mit diejent Den Iruppen,

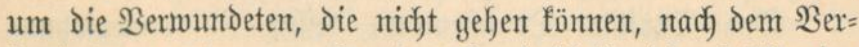

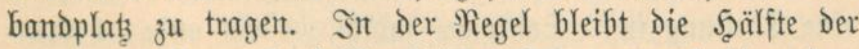
Yerzte und $\mathfrak{L a z}_{z}$ arethgehülfen bei den Truppen, und Die andere Şälfte arbeitet auf dem Berbantoplał̧. Diejer Dient zum 
Zruwpen: $\mathscr{A}$ (nlegent der exften $\mathfrak{B}$ erbände und, falls eine $\mathfrak{B}$ eförberung

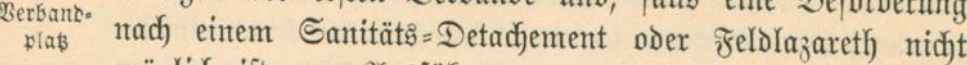

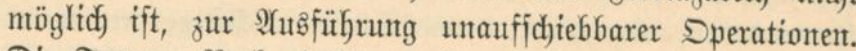
Die Iruppent=Berbanopläbze gehen in Der Regel ein, jobald ein நauptwerbanoplaţ von einem Santitäts= Detadjement er=

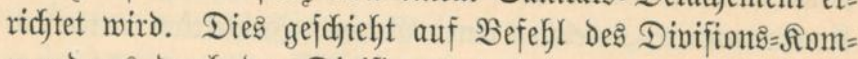
mandeurs Durd) Den Dinifitonsarart.

\section{A. Då Sanitütร̇=Detadjement.}

\section{(\$§ 34-54 und § 202 Der fir.= Sant.= Dront.)}

3wect beฒ Sanitätsి: Detacies ments

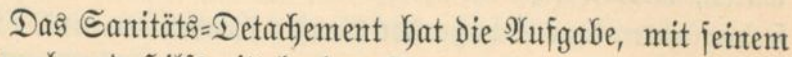
Ferjonal und Şilfşmitteln den Şauptberbanoplats anzulegen,

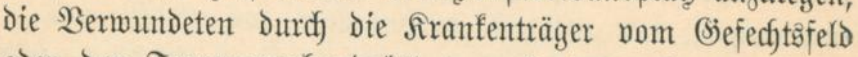
oder Dent Iruppentwerbantoplä̧̧en nad) Dem 5̧auptwerband= plats unto ipäter von bort nad) Dem Feldlazareth zut (d)affert.

2mabibl Derielben

Jedes mobile $\mathfrak{A}$ rmee $=$ Sorps hat 3 Santitäts $=$ Detadje $=$ ments, weldye Beftandtheile des Srain = Bataillonts find und mit fortlaufender $\Re$ ummer interbalb des $\mathfrak{A r m e e}=$ forps be zeidynet merden, 子. $\mathfrak{B}$. Santïütg = Detadjement $\mathfrak{N}$ r. 1 des

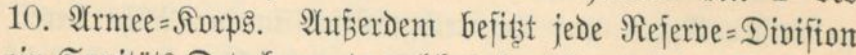

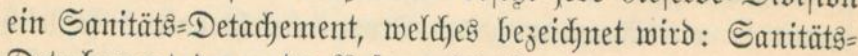
Detadjement Der . . ten Rejerve=Divifion.

Bertheilung

Jeder Infanterie $=$ Divifion wird ein Santität: $=$ Detadje $=$ ment bauend unterftellt, Das britte fteht zur untittelfaren Berfügung des fommandirenden (senterals und wirb der

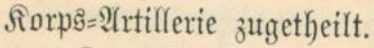

Berional

Das Santäts = Detadjement, Deffen Sujammenjebuntg

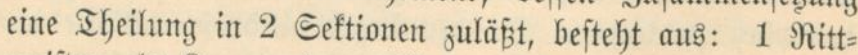
meipter als fiommandeur, Rieutenants, 1 eriten Stabsarjt, Stabs= und Nififtenjärjten, Siranfenträger, J̌elDapothefer, 
Bahlmeitter, Felowebel, lnnteroffiziceren, $2 \mathfrak{a}_{3}$ arethgehülfent, Militärftranfenwärtern unt Irainmamm

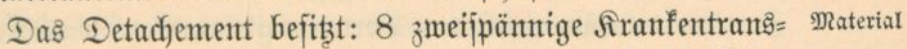
portwagen, 2 żmeijpämtige Sanitätŝmagen, weld)e die $\mathfrak{A}(\mathfrak{p o}=$

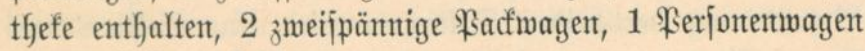
unt 1 Rebengిmittelwagen.

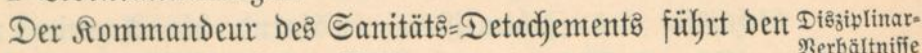
militärijden $\mathfrak{B}$ efehl über dajןllbe.

Heber die $\mathfrak{A}$ erz̧te, Den reldapothefer, bie Razaretbgefhülfent und Militärfrantentmärter hat der erfte Stabsarz̧t interhalb feines Dienftbereidjs die Dişziplinarftrafgemalt eintes nidjt Detadjirten Sompagnie= $=$ She

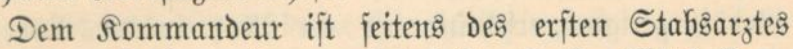

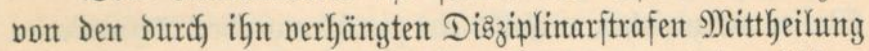
z̆t madjen, ebenjo umgefehrt Dem Reţteren vont Dent feitens Des Eriterent verfügten Dişziplintarftrafent, falls dieje Strafen

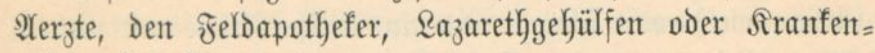
märter betreffert.

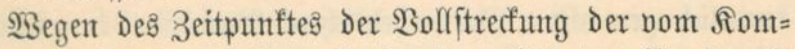
mandeur über Dą̧ leţtere Ferjonal verbängten Strafen ift zur $\mathfrak{S e r m e i d u t a ̣ ~ v o n ~ E t o ̈ r u n g e n ~ i n ~ D i e n f t b e t r i e b ~ D e r ~ e r f t e ~}$

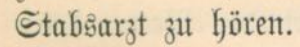

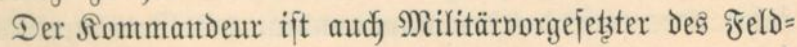
apothefers unt Des 3ahlmeifters unt hat als joldjer die $\mathfrak{b} e=$

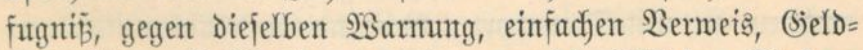
bufe bis ju 9 Mart und, wie gegen Die Dffiziere Stuben= arreft bis

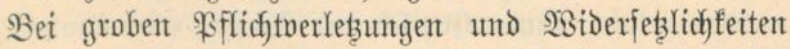

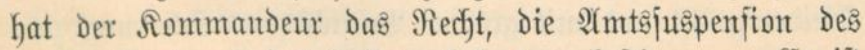

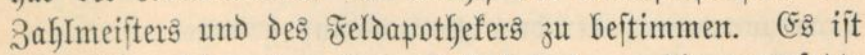
aber ïber jedent Derartigen fall jofort an die vorgeję̧te Snjtant jut beridjtert. 
BertretunB

Die dauternde $\mathfrak{B e r t r e t u n g}$ deษ Feldapothefers und $3 \mathfrak{a h l =}$ meifters ijt bei der reffortmäfigent Behürde zu beantragent.

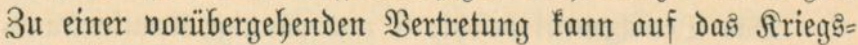
lazarethperjonal zurüfgegangen merden. Bei Der getrentent Berwentung der beiden Seftionen wird eine Seftion Dem Fremierlieutenant unterftellt und Der Felomebel verfiebt die (Siejd)äfte desె 3ahlmeifters, während die $\mathfrak{A}$ rzmeien unter äratlicher $\mathfrak{A}$ (ufficht von einem $\mathfrak{R} a$ arathethehülfen bejorgt merden.

Drt beß

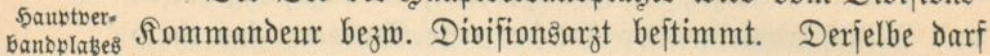

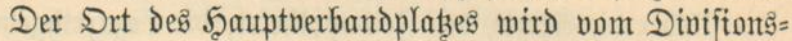

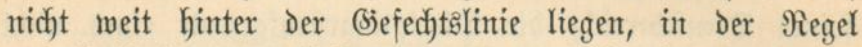
auberhalb des (Siemehrfenters. Went nidjt eit Dorf oder

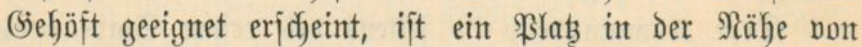
æaffer vorzuzieben. Falls ein zut djururgijdjen Dperationent

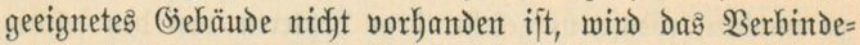
zelt aufgeid)lagent. Der Şauptverbanoplatz wird Durch eine (d) war $z=$ weip =rothe Flagge und eine weife rahne mit rothem firelz, bei eintretenter Dunfellheit Durd) einte rothe Saterne fentlid gemadyt. Bei erheblidjem Borrüđfen der Iruppen

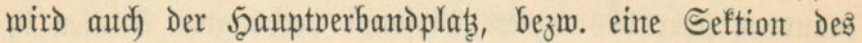
Detadjements vorgejdjobent.

Iranbport nad) bem

5aupt.

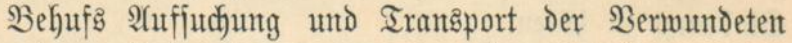
füfrt oder entjentet der fommandeur des Detadjement? die werbanowlas in zmei 3üge unter je 1 Dffizier getbeiltent firantenträger, nad) dem fie mit Irantsport=, Verband = und Sabemittel ver= jehen find, währent und nad) Dem Rampfe auf Das (5ie= fed)tşfeld. Die vorgefundenten marjadunfähigen Berwundeten merdent gelabt, ifnen SSepäcf und $\mathfrak{B a f f e n t ~ a b g e n t o m m e n t , ~ d i e ~}$ Slleiber gelöft und mit gröfter Borfidjt und Sdjomung zum Irantportmagen oder Dem Berbandplał getragent.

Die franfentransportmagen und Die für diejen 3 mecf requirirten mit Strohjojüttung verjebenen Sisagen halten 
zmijd)en der Bjefedfteftelle und Dem Berbantoplaţe, Dem SBagentalteplaze.

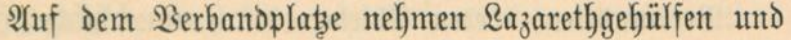

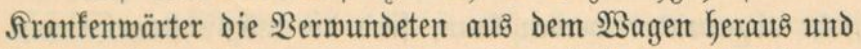
Dienit auf bem bringen fie auf die angemiejenten $2 a g e r j t e l l e n$. Die 2 Gagen verbantwat gehen, nadjoem fie gereinigt, wieder zu neuter ßerwent= buttg vor.

Auf dem Berbandplabe lenft der exfte Stabsarat Des Detadjementรื daร gejammte Sanitätšperjonal unter Der oberen Reitung und naर्d) Den Ŝnmeijungen des Divifiontsarztes. Ex vertheilt dą Perjonal für die äratlidje Behandlung, die Wafferbejd)affung, die Bereitung Der Rabe= und Stärfungg:= mittel. Reb̧teres, jowie derent Berabreidunty ift Sorge des Bahlmeifters oder der damit beauftragten Iraimperjont.

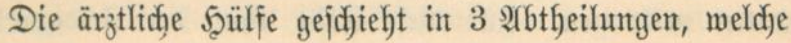
neben eintander mirfen.

Die erite, die Ëmpfangåabtheilung nimmt die $\mathfrak{B e r}=$ Die 3 ab. mundetent in Empfang, unterjuddt, legt frei und reintigt bie

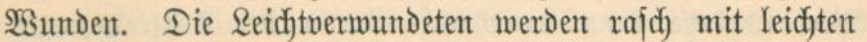
Decfverbändent verjeben uno zunädjit nad) einem, auberballb theilungen DeEิ Rerbanto = Des Săuptwerbantoplałzes gelegenten Sammelplab̧e und fpäter in die Etappenlazarethe gej djift.

Die Sdjwerverwutbeten fommen zur 2. und 3. $\mathfrak{A} \mathfrak{b}=$ theilung.

Die 2., Berbandabtheilung, legt die fodwierigeren und

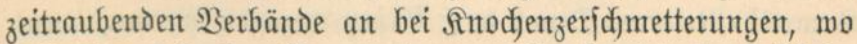
nicht eine jofortige $\mathfrak{A}$ mputation, mohl aber die jorgfältigjte

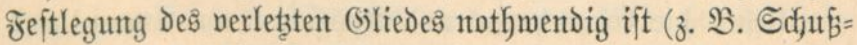
frafturent des Dberjujentels).

Die 3., Dperationsabtheilung, führt die gröfieren dyirux= gijdjen Sperationen ( nidgt aufgejdoben merdent fömen (z. B. Unterbindung 


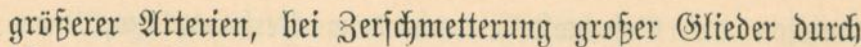
¡d)weres (šejdjüb).

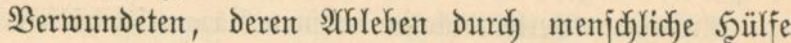
nidgt abgementet werden fant, fint bie möglidfjten (Erleid)= terungsmmittel zit gemähren.

Der Feldapothefer, weldfer unter S(uffiddyt des eritent

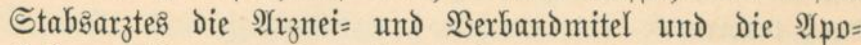
thefengeräthe vermaltet, hat mit dem 3ahlmeifter jeintent

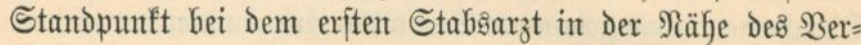

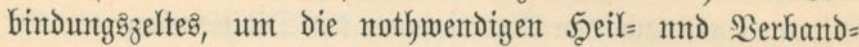

शนtıgabe

Der

5eil= unt

Berbant:

mittel

Eriabs

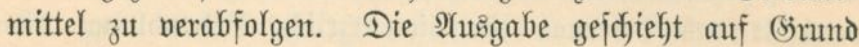

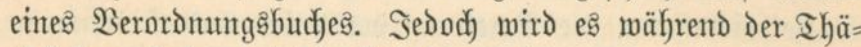

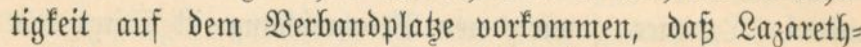

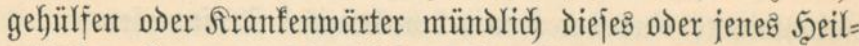
mittel verlangent. F̈̈̈r Dieje Fälle thut der Feldapothefer

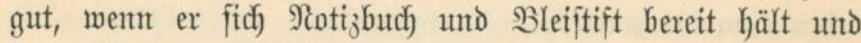

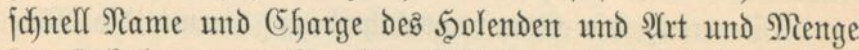

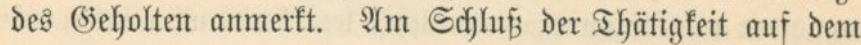

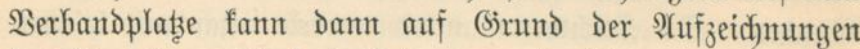

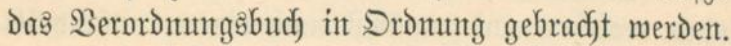

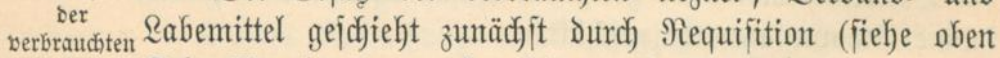
mitter Seite 72) Doder, went fo nidjts zu haben ift, wont Den näd)= ften Razarethen, weldye zut Diejent $\mathfrak{Y}$ bgakent (wie audd) ant

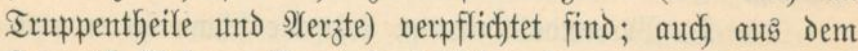

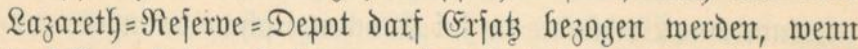
derjelbe mit dent Fafhrzetgent des Detadjements geholt wiro.

theber die Cimnafment und Atuggabent an $\mathfrak{A}$ tzztei= und Serbanomitteln und über Die : Apothefengeräthe hat der Feld =

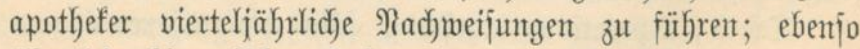
über Die djururgijden Snftrumente unt beräthe, went ifm Die Bermaltung diejer übertragen ift, anterenfalls ijt bies 


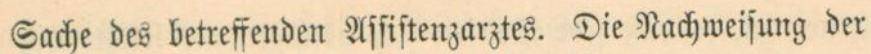
Defonomie=(Seräthe bejorgt Der Zahlmeifter.

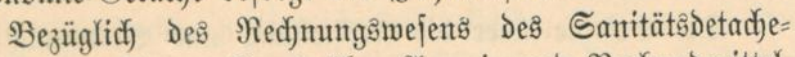

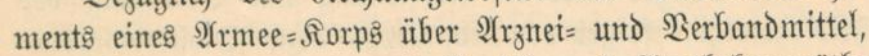

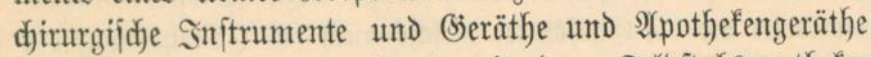

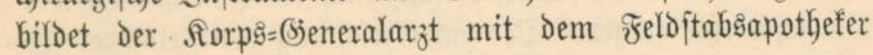

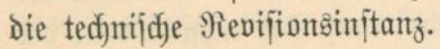

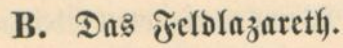

$$
\text { (\$§ 55-100.) }
$$

Das Feldolazareth ift in erfter Rinie dazł bejtimmt, die

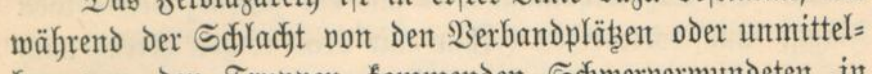
bar von Den Irnppen fommenden Sdfwervermundetent in

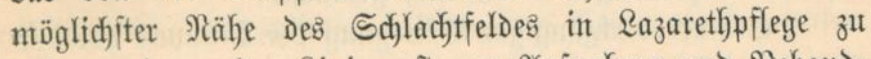
ntehment, in zweiter Sinie erft zur $\mathfrak{Y}$ (ufnafme und Beband= luntg Siranter.

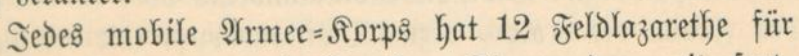
je 200 Berwundete und Siranfe. Sie werden mit fort=

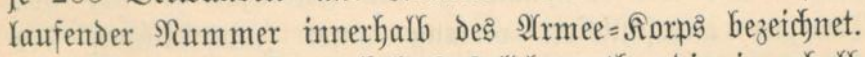

3 wedt Deอ ซelD= lazaretb!

2maabl utto umfang

\$erional

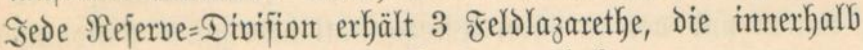
ifrer Divifion fortlanfende Nummern habert.

Das Ferjonal eines zeldlazaretha umfajt: 1 Shefangt,

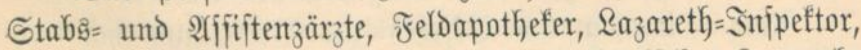

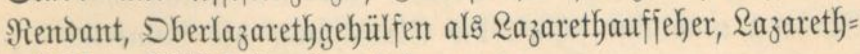
gehülffen, Mitlitärfranfenmärter $\mathfrak{x}$. a.

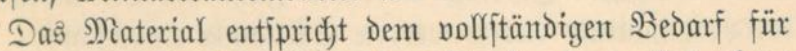
Die genannte Siranfenzahl unt wiro in 2 zmeifpämnigen Sanitätşwagen, in weldy)en fided) die I(pothefe befindet, unt 3 vieripärnigen Defonomie $=$ Mtenfilientmagent fortgejdjafft. Dazu fommt 1 zmeijpämniger Facfmagen (unt) 1 Perjonen=

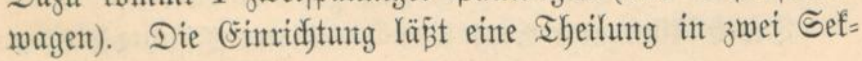


tionent zu, jo dấ jede Seftion fid) nöthigenfalls jelbjtändig auffitellen fant.

Stanbort

nut

Die Befehle über die SAffitelluntg und Bemegung der (Erridtung Feldlazarethe erfolgen von dem forps: bezm. Divifitong:= Iom mandeur an die (Shefärzte, weldhe aud্c) für Intehaltung der Marjobbefehle forgen. Der zur Erridytung der FelDlazarethe beftimmte Drt (Siebäude, (Siehöft) muE fid) möglidjit in ber

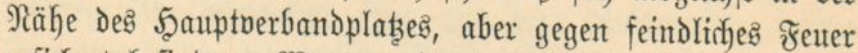
geftchert befindent. WSent geeignete (Sjebäude nidjt vorbandent find, werden 3elte zu je 12 Betten aufgeftellt, die vom $R_{j}$ areth $=$ Rejerve $=$ Depot bejogen werden und weiterfin $\mathfrak{S a}=$

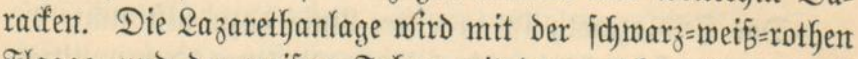
Flagge und Der meifent Fahne mit Dem rothen fireuz aud auf bie Entfernung hin fenntlid) gemadyt.

Rũđวug

Bei einter rüdfgängigen Bemegung Der Truppen hat ber (5hefargt fid) jo einzuridgten, dafis er auf etwaigen Befebl

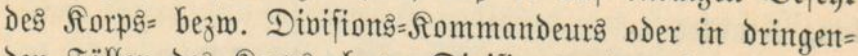

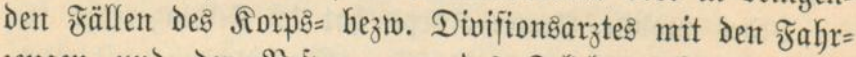
jentgent und Der Bejpantunty des Felolazarethy fidj dent Iruppen anzuljodlefien vermag unter Burüflaffung der frranten und Des zut ifrer Fflege nothmendigften Ferjontals und Materials. Die Feldapothefe dürfte mit Şinterlaffuntg Der nothwentigiten Sceilmittel mitgentommen merden.

(Einrid)tuts

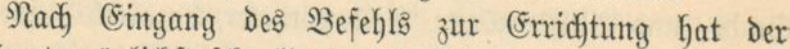

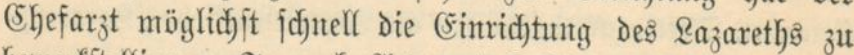
bewerfitelligent. Se nad) $\mathfrak{A}$ rt und Bahl der Reidentoent und der vorbandenten $\mathfrak{Y}\left(e_{3}\right.$ te werden Stationen eingerid)tet.

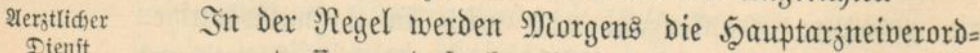
Dienit mutgent getroffen und jo berechnet, daf fie biæ zum Mittage

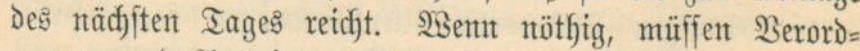
muntgen uto $\mathfrak{A r}_{\text {rzteien }}$ zu jeder Beit gemadjt werdent.

Der $\mathfrak{a}$ ifijtenzarzt trägt nadj beendigter $\mathfrak{B}$ ifite die vom. 
Stationsararte getroffenten $\mathfrak{B}$ eroromungen unter $\mathfrak{B}$ ezeidymung

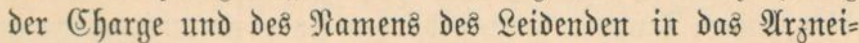

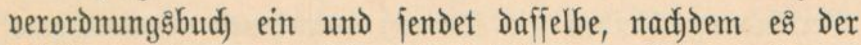
Stationsårąt gegentgezeidjnet hat, jodaun nad) der Feldapo= thefe zur Bereitung der $\mathfrak{A}$ rzznei.

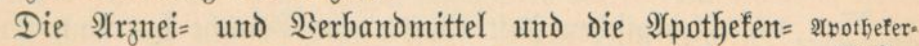
geräthe merden unter Átuffid)t Des (Shefarztes von Dem Feld= bienit apothefer verwaltet. Derfelbe hat die $\mathfrak{N u f g a b e}$, eine vor= ¡d)riftämäbige Zeldonothefe einzuridjtent, weldje des leidyteren

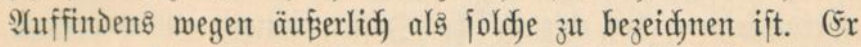
ift ferner für gute Bejdaffenheit und redhtseitigen Erjabs der

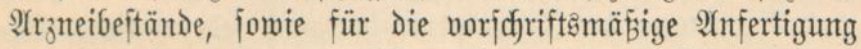

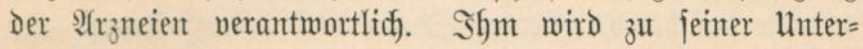
ftüţuntg für die gröberen Dienftleifturgen ein $\mathfrak{A}$ (pothefent= Gandarbeiter beigegeben, weldjer Dem geldapothefer unter= gebert iitt.

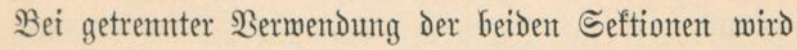
bei der Seftion ohne Feldapothefer die Anfertigung Der

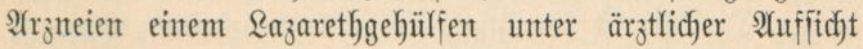
iibertragett.

Die Feldoapothefe des zeld la

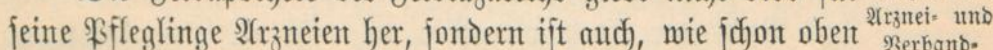
gejagt, verpfliçtet, an bie Iruppent unt Sanitäts = Detadje $=\begin{gathered}\text { Merbants } \\ \text { mitter }\end{gathered}$ ments $\mathfrak{A}$ raznei= unt Berbantomittel abzugeber.

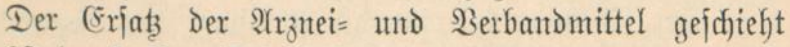
Eriak

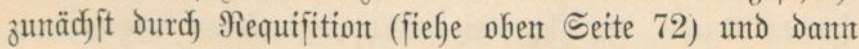

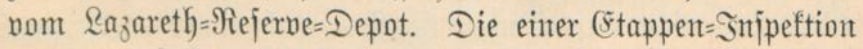

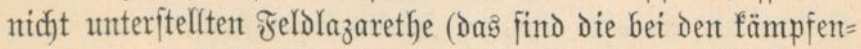

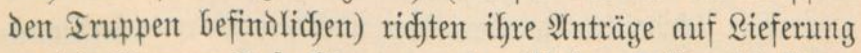

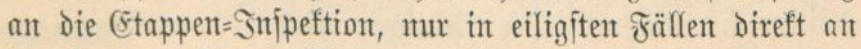

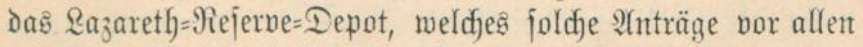
anderen abfertigen joll. Die einer Etappen=Sinjpeftion unter= 
ftelltent Feldlazarethe (joldye, meldye ju weit hinter den fämpfenden Truppen zurüfgeblieben finto, um mit ifnen it Berbindung bleiben zu fönten) entnebment ifren Bedarf Direft vom $\mathfrak{R} a z a r e t h=$ Rejerne $=$ Depot.

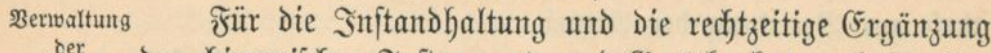

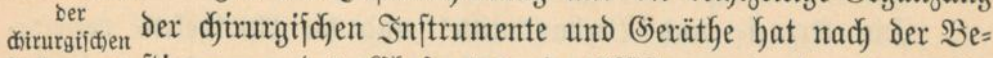

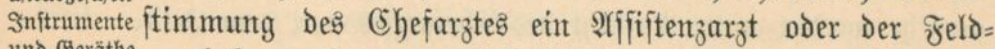
und (Beräthe apothefer ju jorgen, und bringt Der Betreffende nöthigenfall:

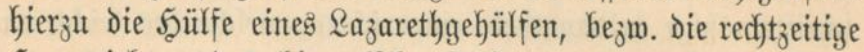
Şeranziefung Des djirurgijdjen Initrumentenmadjers desి $\mathbb{L a}=$ zareth $=$ Rejerve $=$ Depotæ in $\mathfrak{A}$ ntrag. Die jorgfältigite Reinigung und (Entgiftung der Inftrumente hat nad) jedesmaligem (Sie= brauche auf ber betreffenden Station jelbjt ftattzufinden. Die Bermaltung begreift aud die Sorge für deren Berpacfung in fidd).

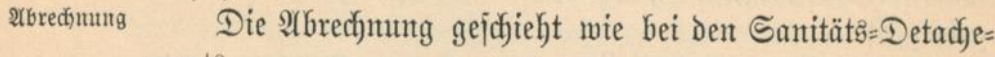
ments.

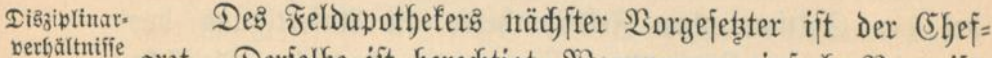

bę̧

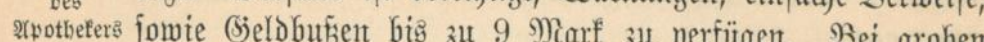
powte Seldbubett big zu 9 Marf ju verfügen. Bei groben PFflidjtwerlebungen und $\mathfrak{B}$ iderjeblichfeiten fteht dem (5hefargt

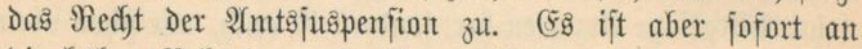
die höhere Bebörde zu beridjtent. SBeitere Diø̈ziplinarjtrafen

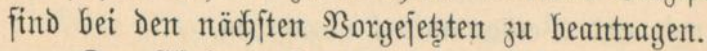

2ตเอินนที

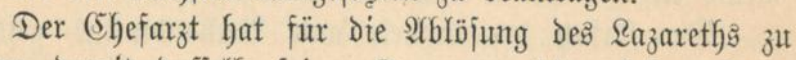
jorgen, damit daffelbe feinen Iruppen mieber folgen fant. (Siebt das nidjt auf einmal, fo find nad) einander bie zwei Seftionen, nie aber fleitere Ibeile freizumachen.

Die Ssebeilten merden der nädjiten Etappentomman= dantur überwiejent. Die Hebrigen merden burd) Bermitte= lung des Feldlazarethdireftors, bezw. Des fiorps = Sienteral= arztę und Der Sranfentransport=Siommiffion entweder in bie 
Sheimath befürdert oder von Den ftehenden ATriegallazarethen aufgentomment.

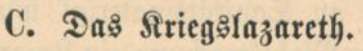 \\ (\$§ 105-108.)}

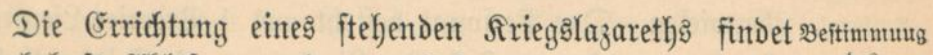

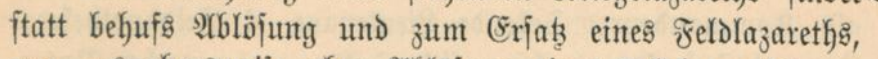

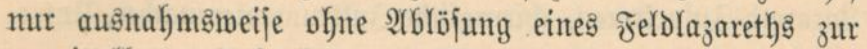
Priegs: unmittelbarent $\mathfrak{N}$ (ufnahme von $\mathfrak{F}$ flegebedinftigen. Sie erfolgt

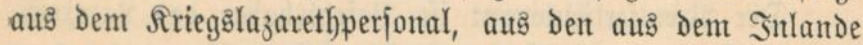
heranzuziebenden bürgerlichen গTerzten nebit sfflegeperjontal unt unter Bemutzutg Der Beftände deş $\mathfrak{L} \mathfrak{z}$ areth $=$ Rejerne= Depots. Sie haben denjelben 3meet wie Die Feld lazarethe, weldhe für ihre $\mathfrak{N}$ (ufgabe bei den fämpfendent Iruppent frei= zumadjen find.

Das̉ \$erjonal bejteht auts Sherftabäürzten, Stabsärzten, Iijititenzärzten, Feldapothefern, $\mathfrak{L a}_{3}$ areth = Snjpeftoren, $\Re e n=$ Danten, $D$ berlazareth $=$ unt $\mathfrak{L a z}_{\mathfrak{z}}$ arethgebülfen u. a. (Fis ift Der Etappen=Snipeftion unterftellt.

So lange das ganze ßerjonal beifamment ijt, ift Der Dižsiblinar.

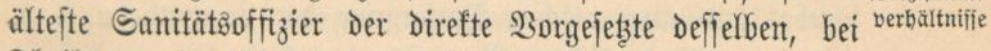
Sheilungen der jedes̊mal rangältefite.

Das Siriegs̆lazareth) hat feine eigene Rafienvermaltumg. Die Nerzte und Beamten beziehent ihre Bjebübrniffe auf Grund Der SAtweijutg Der Etappen = Sntentoantur aus Der

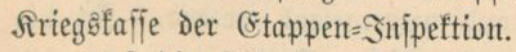

SUf die ftehenden Striegglazarethe finden alle für die Feld lazarethe gegebenten Beftimmuntgen entjpred)ente $\mathfrak{A}(\mathfrak{n}=$ wentoung. 


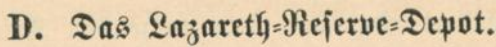

(§§ 109-121.)

Beftimmung 3ur Rejerve an Sanitätsematerial wiro jeder Ětappen= Des̊ \&ajareth: Referve: Devot:

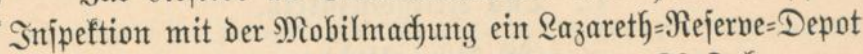

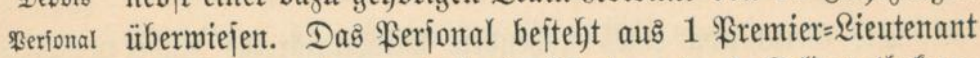
aIs Siommandour, Sefonde= Rieutenant, 4 Fel'dapothefern,

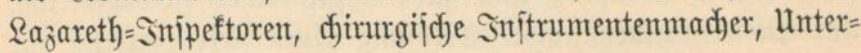
offizierent $\mathfrak{u}$ a.

Dişsiplinar-

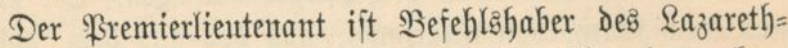
berbăltnifife Rejerve $=$ Depotż. Derjelbe ijt beredjtigt, ïber daz obere Beamtenperjontal 2 Sarmungen, einfadje Bermeije, gegent die djinurgijd)en Injtrumentenmadjer aber auberdem gelindent

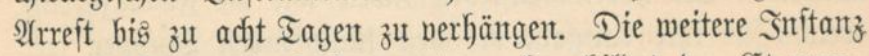

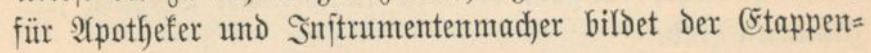
(Siseneralargt.

Berwenbutg

Die Berwendutg der $R$ ajareth $=$ Rejerne $=$ Depots erfolgt

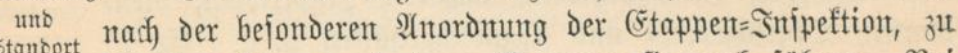
Stantoort

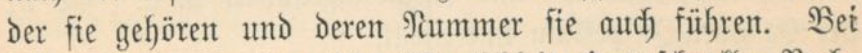

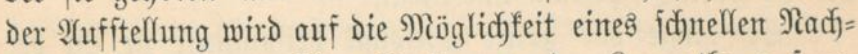

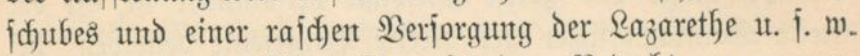
mit Den erforderlidjen Bjegenftäntoent Bedad)t gentommen. Der Standort Deffelben mird in Dent Regel Der Etappen= Gauptort fein, wohin die Beftände des Depotz mit Der Eijenbahn und Der zugeförigent Trainfolonte oder mittelit Borjpant herangezogent und niedergelegt werden. Sit bie Entfermutg des ซeloheeres von diejem Drte z̆ gró, fo wiro Das Depot noer ein Theil deffelfent vorgejdyobent. Dies ge=

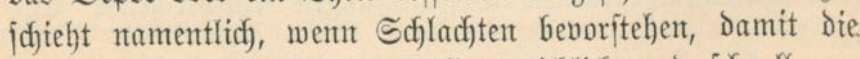
Sorräthe Dent beoürftigen Stellent reidflidf) und fidjtell $3^{\mathrm{u}=}$ gefü̈hrt werdent fömtett. 


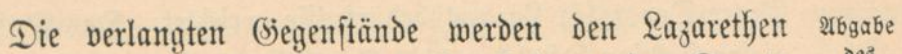

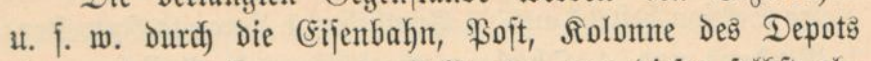
Doer andere Faforzenge jugeftellt oder von diejen jelbjt $\mathfrak{a b}=$ geholt.

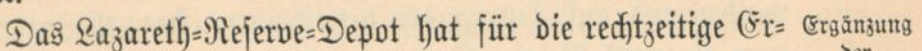
gänzung jeiner Beitände, meld)e (d)leunigit nad) itattgebabter ser YGgabe zu bemirfen iit, zu forgen; eפ bat bezüglidje $\mathfrak{A}(\mathfrak{n}=$

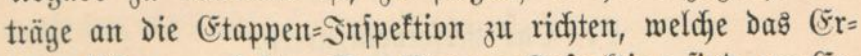

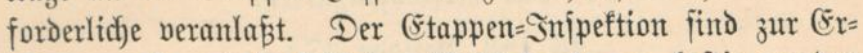
langung einer Meberfidat der Beftände in $z^{\mathfrak{H}}$ beftimmendent Beitabjudyitten Bejtandsberidyte einjureidjen.

Die Auffid)t über bie Durd) die Feldapothefer ftatt= findente $\mathfrak{B e r m a l t u n g}$ Der $\mathfrak{A}$ ranei= und Berbandmittel und Apothefengeräthe exfolgt Durd) Den Damit beauftragten Feld= $\mathfrak{l a}_{j}$ areth $=$ Direftor. Die Derpacfung bejorgen gleid)falls bie Ipothefer.

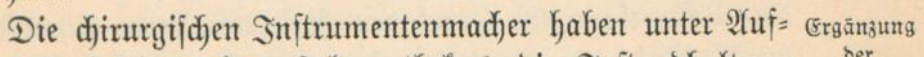

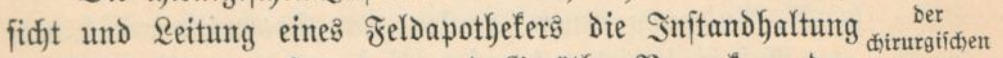
ber djirurgijidjen Snjtrumente unto (Seräthe, $\mathfrak{S}$ Serpacfung Der $=$ Snitrumente felben u. . w. zu bemirten.

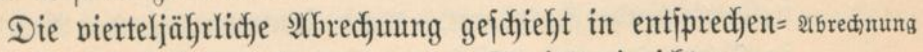

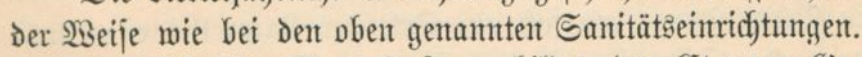
Die ted)nijd)e Mevifions $=$ Snjtanz bildet Der (Etappen $=$ (S)e $=$ neralarat.

\section{E. Då inmobile Ģïter $=$ Depot an cinter Sammelitation.}

(\$ 122.)

San den Gammelitationen, weldye dazu befimmt find,

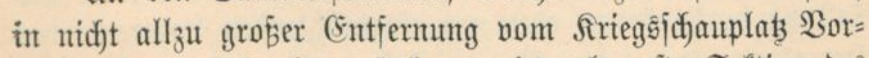
räthe aller $\mathfrak{Y}$ (rt bereit 3 h halten, wiro als erite Seftion Des

3wect De8 Depots 


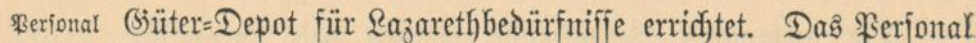
biejer Seftion bejteht aus: 1 Ipothefer, $1 \mathfrak{L} \mathfrak{a}_{z}$ areth $=$ Snjpeftor, 1 Rendant und 4 Dherlazarethgehülfen als $\mathscr{A}$ uffeher, und hat die in Dem Depot niedergelegten Biegenfitände zu ver= malten und auf $\mathfrak{A}$ trag ber betreffenden Etappen= Injpeftion Dem $2 \mathfrak{a}$ areth $=$ Iejerve $=$ Depot zuzjendent.

\section{F. Då $\Re$ ejervelazareth.}

\section{(§ 183 Beilage 47.)}

Begriff bes Reierve: Iazaretba Bernumbeten und firanfen werden im Inlande zu Den por=

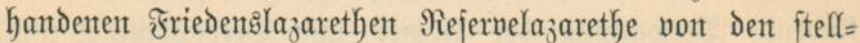
Beiontere Beftim. numben vertretenden Behörden erridjtet. Diejen Ramen fübrent vom 1. Mobilmadutgatage ab aud die Friedentalazarethe, mit

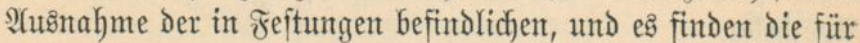
die Rejervelazaretbe gegebenen $\mathfrak{B} e$ fimmungen $\mathfrak{A}$ (nwentoung (3. B. Afnlegung neuter firanfent= und Iodtenliften u. j. w.).

Wo eit Ipothefer anz่̧นtellen ift

QYuf je 400 Sirante wird ein $\mathscr{Y}$ pothefer geredfnet. Daß mirflidye Bedürfnifi interbalb diejer Sirenze beftimmt Der

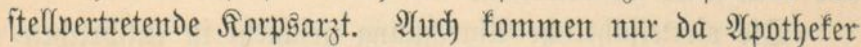
in Bermentung, wo fich am Drte des $\Omega_{3}$ areths eine aus= reidjende Dijpenfitanitalt befindet. $\mathfrak{S}_{0}$ eine foldje nidjt vorhanden ift, müffen die nöthigen $\mathfrak{N}$ (rżeien dispenfirt aus Dent Stabtapothefent entnomment werdent.

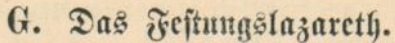

Begriff be Jeitungs: 2aarath

Bei eintretender 9)iobilmadjutg erfalten alle in der Fejtung vorfandenten ober neu einzuridytenden $\mathbb{L} a$ aretbe der Sceres̄nermaltung Den Namen Feftutgslazarethe und geltent

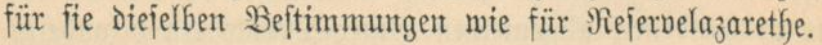


Wind eine Feitung armirt, fo geht die Reitung des ge= Beiontere fammtent Sanitätädienjtes auf Den (Sarnijonarzt über. Der=

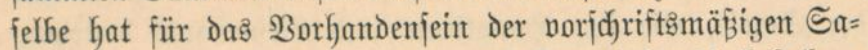

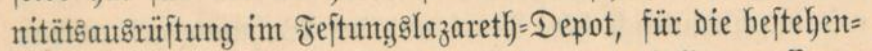
Den Razarethe und Die für Den Belagenutgĝfall getroffenten \&azaretheintidytungen bezm. Derent Şerftellung zu forgen unt bie Reitung Der Razarethe und Depotvermaltungen zu über= madjen.

\section{Dex Stuhanpothekre.}

Die Stabsanothefer bildoen gegenüber den jährlidu wedf)= felnton einjährig=freimilligen Spothefernt und Den Ipothefern Des Beurlaubtenftandes den feitftehenden Stamm der Şeeres = apothefer, Deffent Spitze Durdd) Den Dberitabsanothefer gebilidet wird. (ES fint ifrer $18 \mathrm{im}$ Dentjdjent Seere, bei jedent 2 sirfumb. Atrmeeforps eiter, der Rorpsiftabşapothefer. Derjellbe hat unter Dberauffidd)t Des Siorpsggeneralarjtes Die perjönlidjent freis Der Gtabs:

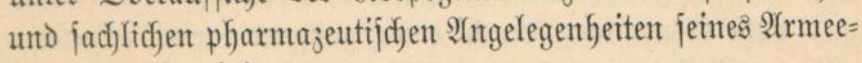
Siorps jut bearbeitert.

Die forpsftabsapothefer werdent aus Den Şeereşapo = sinberufums

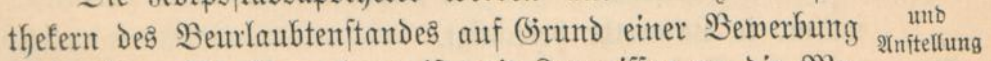
einberufent. Die Bewerbung ift mit Bentgniffent an die Mie=

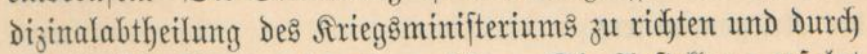

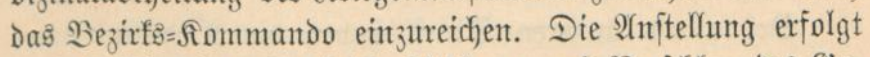

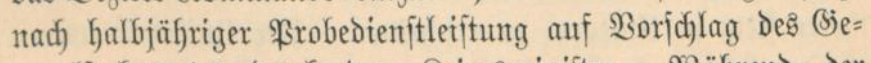

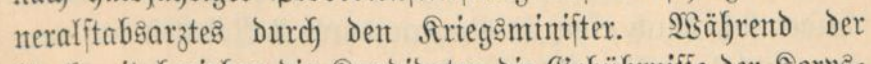
Frobejeit Geziehen die Randidaten die (3ebüfrniffie der אorps: ftabsapapothefer.

Die Sorpâtabasapothefer fint auf Rebenszzeit angeftellte, pentinisıberedytigte, bejoldete, obere Militärbeamte, Die auker

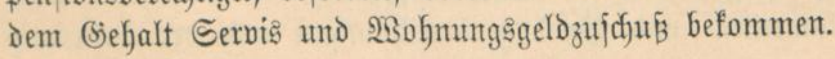


Daş (Siehalt beträgt mindeftent 1800 Marf und höd)=

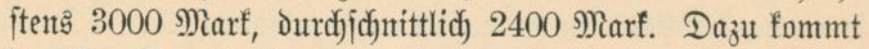
Der Servis, wie inn 5̧auptlente und Rieutentants bejiehen,

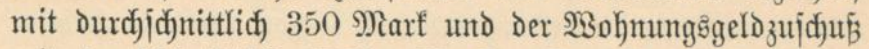

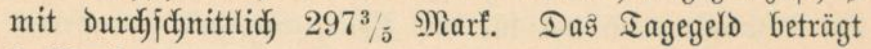
9 Miarf.

Die Berbältniffe der Rorpşitabsapotheter in Bezutg auf Den Rang, die Begrübung jeitent der Mamnjodaftent unto Unteroffiziere, die allgemeinen Strafbeftimmungen, Den $\mathfrak{B} e=$ id)merdemeg und die Dffizierranglifte finto gleidy denjentigen Der Dberapothefer.

Derere

Sutitanzen

Łtraub

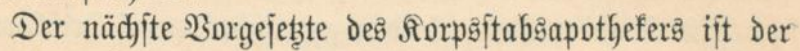
Sorpsgeneralarzt, Dem er attadjitt ijt. Bont diejem gehent z'mei Snitanzenwege: Der eine zum fommandirenden Bjenteral und Dant zum friegsminifter, und der andere zum Bjeneral= jtabsanarzt.

Itring

$1^{1} /$ Monat zu ertheilen. Eine nothmentige Bertretung wird vom (sieneralargt burd) einen geeignteten einjübrig=frei= willigen Millitärapothefer angeordnet.

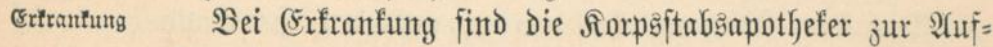
nahme in $\mathbb{R} a z^{2}$ arethe beredftigt.

Ber. $\quad 3$ werkeiratbung bedürfen diejelbent der (sientefmigung deติ Siriegsmminifterต.

uniform Bei allem äub́eren Dienjt haben die Rorpştab̧apo= thefer ftets in Uniform zu exjobeinen. Die Uniform unter=

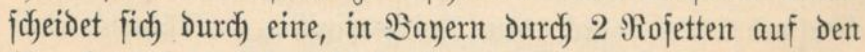

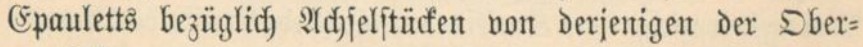
apothefer.

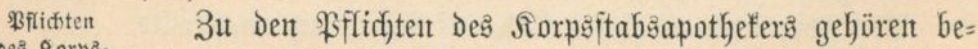

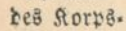
apotbefers

jonders: die Revifion der vierteljährlidłen tabellarijuden Mie= Difamenten = utd Bandagenbered)nungen und Der bazu ge= 
Görigen Riquidationen Der Drogiften, Fabrifautent und Âpo= thefer,

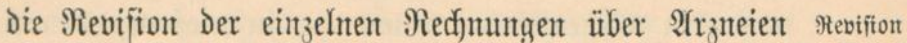

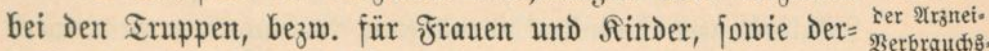
jenigen, weldje für Detadjirt jtehende Feldowebel u. j. w. von beredt: Sivilär ${ }_{j}$ ten verorònet finto.

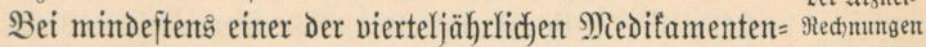

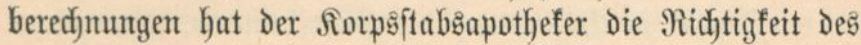

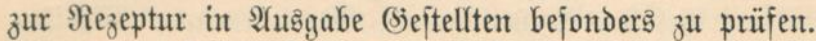

Ferner hat derjelbe die Ėmpfantgasbej deinigungen bejw.

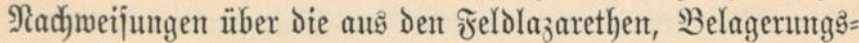
lazarethen, Irain=Depots, Sorpsąarneireferve it. a. an Die Dijpenfiranjtaltent und $\mathfrak{A r}_{3}$ neirejerve abgegebenten $\mathfrak{Y}_{r_{j}}$ neient,

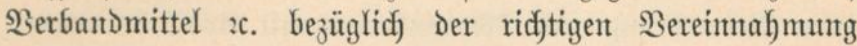
Derjelbent zat prüfent und zut bejdeinigent.

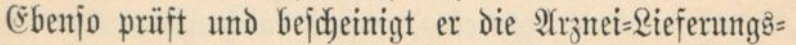
verträge für die Dijpenfiranjtalt, Die Redfmungen über Die

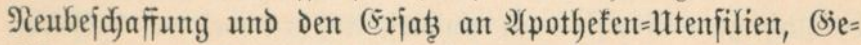

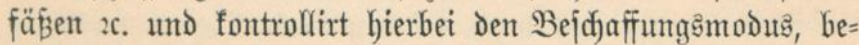
fonters aud die Ingemeffenheit der \$reije.

Studf) liegt ifm die Früfung der Riquidationen über d)emijd)e Unterjudfungen ob.

Die revidirten unto atteftirten Red)nungent werdent Dem תorpsigeneralarat zur Fefititellung vorgelegt.

In Betrefi Der \$erjonalien Der Militärapothefer feines

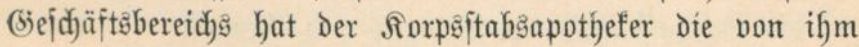
zuvor geprüften Bettgniffe bei $\mathfrak{A}$ (ntmelountg der einjährig=frei= willigen Millitärapothefer Dem Rorpsgeneralar ${ }_{3}$ t vorzulegen, Arzmei= Sieferung 刃erträge Die Rorrejpondent 2 z. Gezüglid) Der Saftellumg und Entlaffutg, fowie die Riftent über Die angemeldeten jowohl als aud über

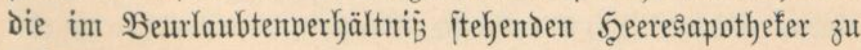
fübrent.

Riftelts fübrung ber Seeress: apotheter 


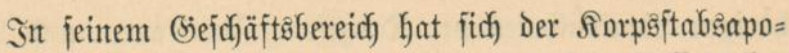
thefer über die beften $\mathfrak{B}$ ejuggiquellen aller fein Refifort be= treffendent Biegenftä̈toe gentau ju informiren, damit den Eofalinitanzen hierüber die näheren sseijungent ertheilt wer= Dent fömtrett.

Revifitin

ber

2ranei-5inriditungen

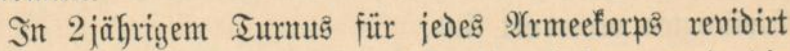
derjelbe die Dijpenfiranitalten, die $\mathfrak{A r}_{3}$ neirejerve unt die Irain=Depots in Bejug auf die Medifamente, Spothefent=

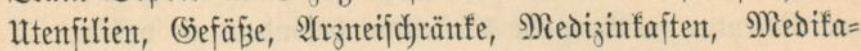

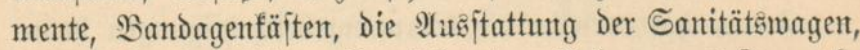
jowie Die bejüglidjen Sorräthe Der Belageruntg $=2 a_{3}$ areth $=$ Depots.

Die Irain $=$ Depots und diejenigent Irntppentheile, in

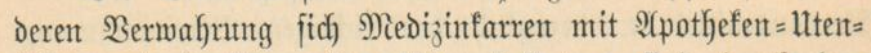
filien und jonftigent Requifiten befinden, fint dutrd dent

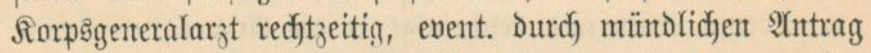
bei Der Meldutg beim Sarnifon= Sommantbanten von ber

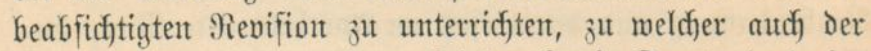
betreffende Iruppentarat bejw. Gei den Irain=Depots Derjenige Dhermilitürarż mit heranjusiehen ift, Der Gei Der Bejd)affuturg Der Getreffenten Requifitent fïr daffellhe fontutrirt.

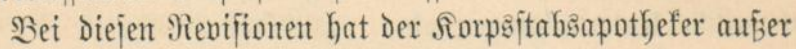
Der Frififuntg des Soll= und Sitbeftandes und der allgemeinent

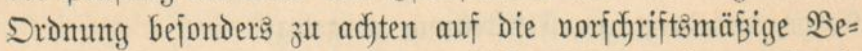
fidaffentheit ber Medifamente, auf ifre $\mathfrak{Y}$ (ufbewahnutg, fowie auf Die Bejd)affentheit Der 2 saagent unt (semid)te.

Nur erwiejenermafent untrautdybare Medifamente finto jofort ju bejeitigert, forrft hat fidj) Der Stabsapotheter jeder

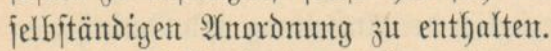

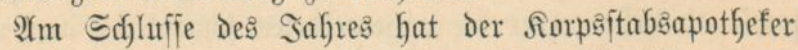
feinem Benteralarjt über jeine Berwaltung Beridgt jut er= ftatten und Daran Sorjajläge jur Bejeitiguty der etwa be= 


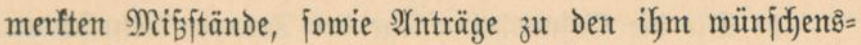
merth jodjeinenden Berbefijerungent zu fnüpfen.

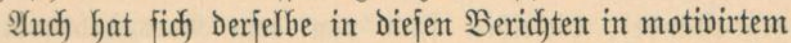

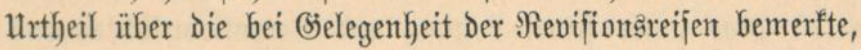

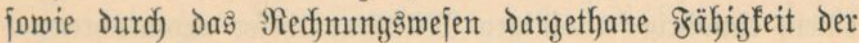

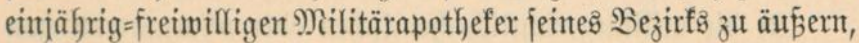
namentlid) für ihre eventuelle $\mathfrak{B e r m e n d u n g ~ a l s ~ f t e l l v e r t r e t e n t o ~}$

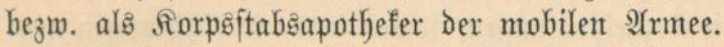

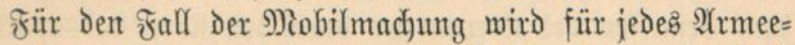
forps dem ftellvertretenden \$rovinzial=(Sieneralarat ein ftell= nertretentoer Rorpsitabsapothefer zugetheilt, Der nad) ent= ipredjenden Beftimmungen, wie fie für Den Frieden vor= Stell. vertreteri ber Siorts: apothefer gejdyrieben find, für die Bejdaffung Der das pharmazentijdde ซad) betreffenden (Siegentäntoe für das mobile Sceer, die

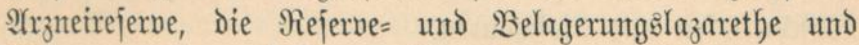
Belagerungglazareth= Depots Sorge zut tragen hat.

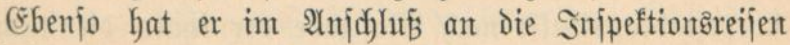
Des ftellwertretenton Frovinjial = Sienteralargtes die Dijpenfir=

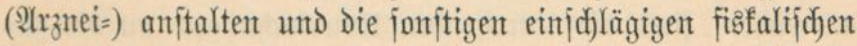

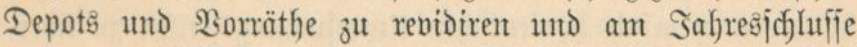
bezm. am Sdjluf jeiner dienftlidjen Ibätigfeit jeinem näd)= ften Borgejesten, Dem (Sienteralarat, einen bejonderen Beridjt itber diejelbe einzureidjen.

Im Ulebrigen geltent für die ftellvertretenden Siorpsapo= thefer diejelben Beftimmungen, wie für den aftiven forps:

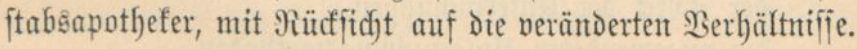

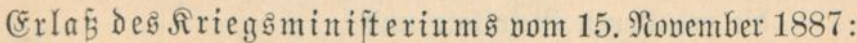

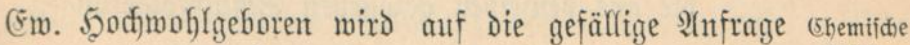
ergebenjt ermidert, dấ ju Den Dienftlidyen Dbliegentheiten Der forpsitabsapothefer Die Afusfüfrung folgender Unter= unter. futdungen fudjungen gebört: 
1) Die bie Biejundheits̊pflege der Truppen betreffendent

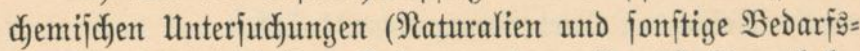
gegenftände); Diejelben find von dem fiorpsittabs̊apothefer jelbjt oder unter feiner Reitung und $\mathfrak{B}$ erantwortlidjfeit von

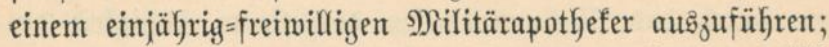

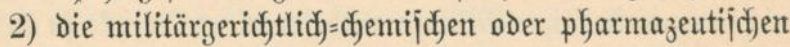
unterjudfuntugen;

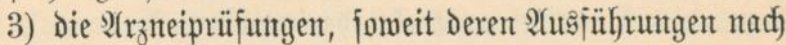

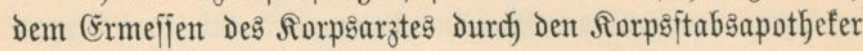
erforderlidid jojeint;

4) Diejenigen djemijanen und mifroffopijden unter=

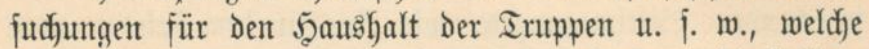

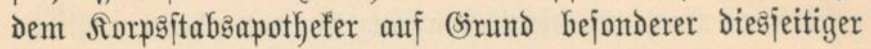
Berfügungen übertragen merden, wie bie Unterjud)ung vont

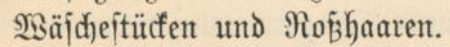

Die vorfteheno bezeidjneten Unterfudjungen habent die

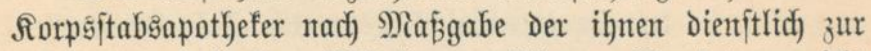

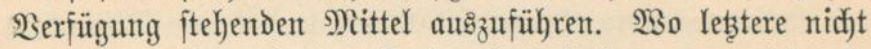
ausireid)en, find bie betreffenden unterjudungen an bie ju= ftändigen d)emij weijen, eventuell entipredjente $\mathfrak{A n t r a ̈ g e ~ h i e r h e r ~ z u ~ r i d y t e r . ~}$

Die von Dem Rorpsftabsapothefer auf Grumb Der auts= geführten Unterjudfungen zul erftattenden (ऊ)utad)ten fint jo $\mathfrak{a} \mathfrak{b}_{z} u \mathfrak{f} a$ ffen, Dá̧ bei einer etwaigen Sorlage hier

Der Zmedf Der Unterjutdungen,

bie angewendeten unterfuddungsimethodent,

die erhaltenen Rejultate unt

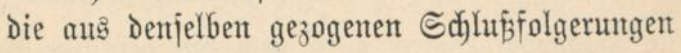

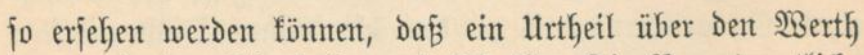
ber ermittelten Thatjadjen möglid) ift. Die Serantmortlid)=

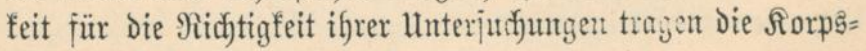


ftabşapothefer allein und haben fie demjulfolge die betreffen= Den (sittadyten allein zit unterzeid)nett.

Eine 3ujammentellung der im Raufe des Getreffenden

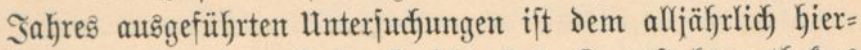

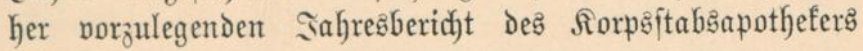
beiz̧ufügen.

Sirieggeminifiterium. Ned.=A(bth).

\section{Bar (D)erfabsapothrker.}

Einen Dberftabsapothefer giebt eร mur in Freufent und fier audd nur einen einzigent. Derjelbe gehört $j^{\mathrm{u}}$ Der $\mathfrak{M}_{i}=$ Wirfunถ freiß

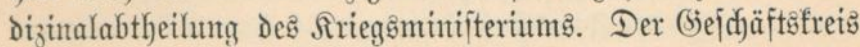
Der Nedizinalabtheiluntg umfaít das gefammte Şeeres: janitätsomejert. Daju gehört unter anderen die Serjorgung

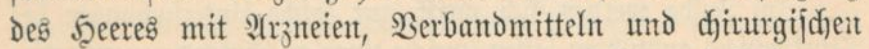

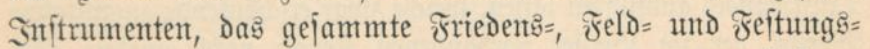

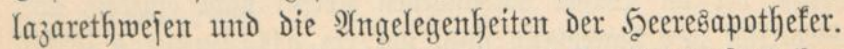

Die genantent 3meige des Seeres:=Santitätsmejents bear= beitet Der Dberftabsapothefer zur $\mathfrak{B}$ orlage für Dent (jieneral=

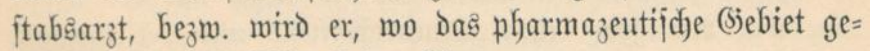
Bflid)ten deฐ Dberitabs. ftreift wird, als Mitarbeiter hingugezogent.

Der Dherftabsanothefer gehört jut Dent Sivillbeamten der ઉebübruiffe

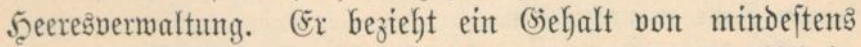
3000 Marf und hödjitens 5400 Marf, im Durddjidnitt

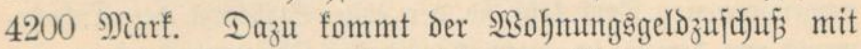
900 Marf. Das Tagegeld beträgt 12 Marf.

Die Nangoromutg für (Sivilbeamte vom 7. Februar 1817

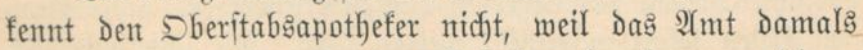
nod) nidjt bejtand. Sit Dent (Sebaltsinadjweifungent wird er Den Ied)nifern und Referenten zutgezäblt. 


\section{$\mathfrak{d} \mathfrak{n} \mathfrak{j} \mathfrak{a} \mathfrak{i} \mathfrak{g}$.}

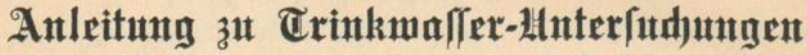 int fidite.}

(Nađ) Der Siriegs=San.:Dronung v. 10. Sant. 1878.)

\section{Grundiäze.}

Ĵm Felde werben fich meijt mur joldfe Itnterjuchungen beక Trintwajjers ausfübren Iafjen, welche zwar exjojöpfende quantitative Werthe ber im Wajfer vorhandenen Siörper nidgt ergeben, aber erforberlid) und hinreichend find, um ein allgemeines Utrtheil über bie Brauthbarfeit oder $\mathfrak{U n b r a u c t b a r f e i t ~ D e f f e l b e n ~ z u ~ b e g r u ̈ n d e n . ~}$

Die Ilnterjucthungen werden in Der Regel mur bei länger Dauernder Bemubcung einer Trinfwafferftelle und bei längeren Santonnirungen u. f. w. ftattfinden, find Dann aber, wenn irgento

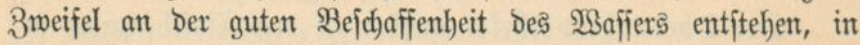
feinem Falle zu unterlajןen.

Die Unterjutchungen merden bei Den Sanitäts = Detachemenţ vorgenommen. Dort läpjt fie Der erite Stabsarzt Des Detaches ments von Dem Feldapotbefer, Defien Dbliegenbeiten beim Sa=

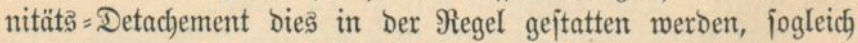
auşü̈hren, Damit Das Ergebnip Dem Ueberjender Der \$robe Durch Den Boten fofort mitgetheilt merben fann. Die Unterjutbungen erforbern ifrer Natur nach im IIfgemeinen einen Zeitaufmand biş ž etwa einer Stunde. 


\section{Reagentien un๖ Geräthe.}

Bei jedem Sanitäts=Detachement befindet fïch ein Reagentien= fajten zur $\mathfrak{B a f j e r u n t e r j u d f u n g ~ m i t ~ n a d j j t e h e n d e m ~ \Im n t h a l t : ~}$ Эnbalt:

a) Reagentien in Flajchen mit (Slaştöpjel von $100 \mathrm{ccm}$

1) Saliumpermanganatlöjung $(0,316 \mathrm{~g}$ im Liter $=1 / 100$ normal). Da bieje \&öjung in ifrer 3 ujammenjeb̧ung nad) längerer

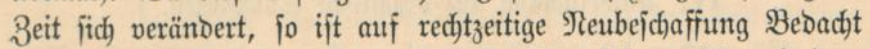
zu nebmen. Daffelbe gilt von Der Draljäurelöjung (3) uno Dem

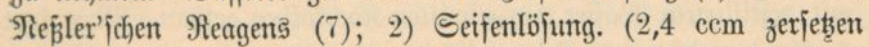
$8,8 \mathrm{mg}$ (alciumcarbonat); 3) Draljäurelöjung $(0,63 \mathrm{~g}$ im $2 i t e r$, $1 / 100$ normal); 4) Sialiumdfloriolöjung $(0,03 \mathrm{~g}$ (Ehlor im \&iter).

In Flajdjen von $50 \mathrm{ccm}$ Inhalt:

5) Sd)wefeljäure von 1,840 jpez. (Sem. (jalpeterjäurefrei); 6) Sal=

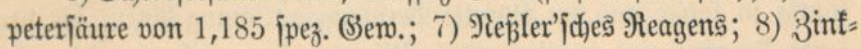
jobiojtärfelöjung; 9) Silbernitratlöjung $1: 20$; 10) Ammonium= c)loriolöjung $(0,004$ Immoniaf im Riter); 11) Raliumnitritlöjung $(0,0024$ im ¿iter); 12) Brucinlöjung (1:800 bejtill. Wßajier); 13) Salpeterlöjung $(0,04 \mathrm{~g}$ im Qiter).

b) Bseräthe:

14) ein Rochfolben von $150 \mathrm{ccm}$ Snhalt; 15) ein (SIa gefäp̄ (Stehcnlinder mit (Slaşłtöpjel) von $75 \mathrm{ccm}$ Snhalt mit Marfe bei $40 \mathrm{ccm} ; 16$ ) eine Iropfbürette (Fூndrotimeter) zur Scärtebeftimmung; 17) eine Iropfbürette von $10 \mathrm{ccm}$ Jnhalt;

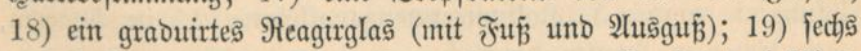

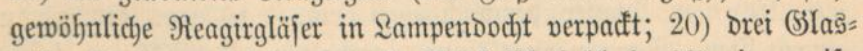
ftäbe (einer zugejpint); 21) ein Stüct \$latinblect);22) eine weipe

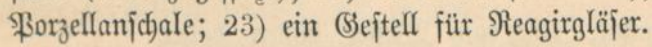

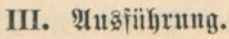

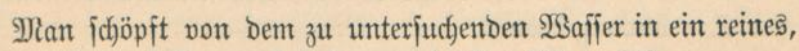

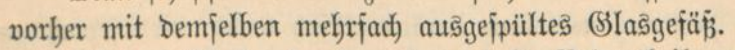

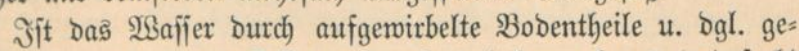
trïbt, fo muip jid) dajfelbe erit gejebct haben, ehe es unterjucht wirb; Filtriren bejolleunigt die silärung. 
Seat jith Das Irintwajfer nidft bereits burd) Die gewöbnliche Prüfung mit Den Sinnesorganen als unbrauchbar ermiejen, jo folgt bie chemijace Unterjuchung.

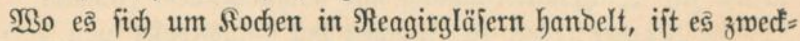

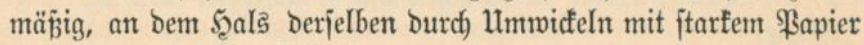
eine Seanbbabe herzujtellen.

\section{Itnteriudung auf organiide Subitanyen. ')}

Es werben $50 \mathrm{ccm}$ Wafjer abgemefien und in ben jorgfältig mit bejtillirtem $\mathfrak{W a j j e r}$ ausgejpülten Rocbtolben gethan. Wian jeşt $3 \mathrm{ccm}$ verðünnter Sd)wefelfäure $(1 / 2: 2)$ und vermitteljt Der Bürette $5 \mathrm{ccm}$ Raliumpermanganatlöjung hinz̧u. Allsbarm fodft man 10 Minuten lang, gię̧t nach) bem Rochen $5 \mathrm{ccm}$ Draljäure hinz̧u, fchüttelt bỉ zur Entfärbung und Rlärung, tröpfelt vor= fichtig jo lange aut Der Bürette Fermanganatlöjung hinzu, bỉ ber leb̧te Iropfen eine menigitens 5 Mimuten lang itehente, idfwache Rotbfärbung bewirft.

Die Summe ber verbraudbten cem \$ermanganatlöjung wentiger Der angemandten ecm Draljäureröjung ergiebt Die Menge \$ermanganat, weldye zur Drybation ber organijchen Subjtanzen erforberlich war.

Beippiel. Âtrgemanot fino: $\mathfrak{W a f j e r} 50 \mathrm{ccm}$, binzugefügt Sialiumpermanganatlöjung $5 \mathrm{ccm}$, Draljäurelöjung $5 \mathrm{ccm}$, nad) Dem Siochen Fialiumpermanganatlöjung $2,5 \mathrm{ccm}$. Demnach ver= braudbt: Saliumpermanganatlöjung $=5+2,5=7,5$, Draljäure= löjung $5 \mathrm{ccm}=5$, Saliumpermanganatlöjung 2,5 in $50 \mathrm{ccm}$, auf $100 \mathrm{ccm}$ Wajfer Demuad) $=5 \mathrm{ccm} .10 \mathrm{ccm}$ Der genau eingeftellten \$ermanganatlöjung entbalten $0,00316 \mathrm{~g}$ fejtę Saliumpermanganat, $100 \mathrm{ccm}$ Wafjer haben baher $\frac{5 \cdot 0,00316}{10}$ $=0,00158$, oder 100,000 I heile 1,58 Theile Sialiumpermanganat zur Drybation gebrautht.

Die Sermanganatlöjung verändert jich) Ieidjt, wirb jebod) $D a=$

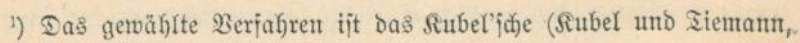
pag. 104-106). 
Durch) nicht voll(jtändig unbrauc(b)bar; man hat nur burch einen mit Der bejtänoigeren Draljäurelöjung anzujtellenden Berjuct) ifren Sebalt an unzerjeb̧tem Raliumpermanganat von Neuem zu er= mitteln. $3 \mathfrak{u}$ Dem Ende erbib̨t man $45 \mathrm{ccm}$ Wajjer mit $5 \mathrm{ccm}$ ber Draljäurelöjung biß z̧um Sieben, jeşt ßermanganatlöjung

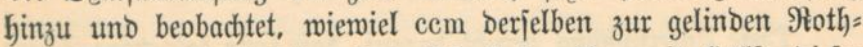
färbung ber Flüjfïgfeit erforberlich find. Utm mit Şülfe biejer ¿öjung von etwaß̉ verändertem Behalt sie Theile feíten Salium= permanganats zu ermitteln, weldye zur Drubation Der organij(j)en Subjtanzen in 100,000 Theilen Wajjer erforberlich find, zieht man von ber Sejammtmenge Der bei Dem Berjuthe binzugejeţten ccm \$ermanganatlöjung Die zur Drnbation von $5 \mathrm{ccm}$ Der Dral= fäurelöjung erforberlidjen ccm \$ermanganatlöjung ab und mul= tiplizirt bie Differenz an ccm mit $2 \cdot \frac{0,00316}{\mathrm{x}}$, mobei x bie ccm \$ermanganatlöjung bezeidgnet, weldbe $10 \mathrm{ccm}$ Draljäurelöjung ent= iprechen.

\section{Interịud)ung aแ̣ Galpeterīüure. ")}

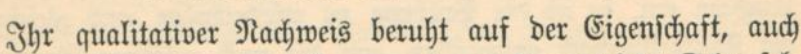
bei grof̧er Berbünnung in Segenmart von fonzentrirter Schmejel=

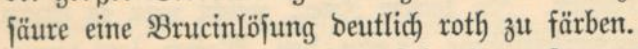

Man führt die \$rüfung folgendermaß̧en aus: 3 Tropfen besె zu prüfenden $\mathfrak{W a f f e r s ~ b r i n g t ~ m a n ~ i n ~ e i n ~ m e i f ̧ e s ~ \$ o r z e l l a n j i c h a ̈ l = ~}$

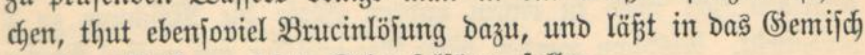
tropfenweije fonzentritte S(d)wefeljäure fallen.

J̃it Salpeterjäure in erbeblicherem Map̉e vorbanden, fo wiro bie Flüifitgfeit Deutlid) roth gefürbt.

3ur Sontrolle verjeţt man 3 Iropfen ber Salpeterlöjung in gleicher $\mathfrak{W e i j e ~ m i t ~} \mathfrak{B r u c i n l o ̈ j u n g ~ u n d ~ f o n z e n t r i r t e r ~ S c h m e f e l j a ̈ u r e . ~}$

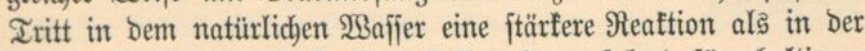
Berjudb) flüjfigfeit auf, fo ijt bajfelbe als zu jalpeterjäurebaltig zu bentanden. Die fonzentrirte Sdywefeljäure ijt vor jedem $\mathfrak{B}$ erjuct)

1) Das vorgejudriebene $\mathfrak{B e r f a h y r e n ~ i j t ~ g e n a u ~ b a s ~ R e i d j a r b t ' i d j e . ~}$ 


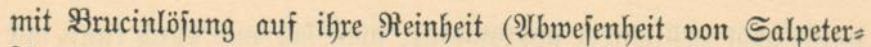
fäure) zu prïfen. Erforberlichenfalls ijt bie vorbandene Salpeter= füure Durch) (Errwärmen $z^{\mathfrak{u}}$ entfernen.

\section{Unteriudung auf jalpetrige Säure.}

Dieje Unterjud)ung beruht barauf, bá̉ freie falpetrige Säure

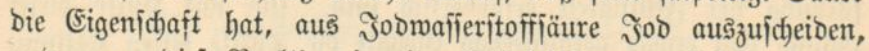
und, wenn bieje Reattion in einer Stärfelöjung vor fich geht, bieje

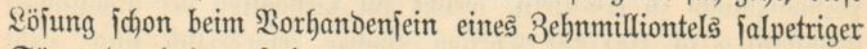
Säure Durch das freimerdende รod zu bläuen. Man vermeide

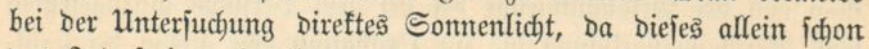
Das Эod frei machen fann.

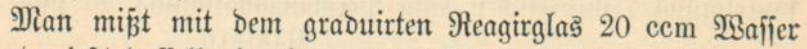

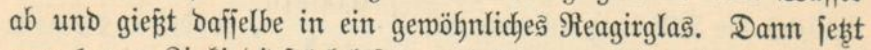
man $1 \mathrm{ccm}$ 3infjobiojtärfelöjung und $1 / 2 \mathrm{ccm}$ veroünnter Sdjwefel= fäure binzu, joüüttelt um und beobachtet Die Farbenveränderung. Bildet fich ein bunfles Blau, fo ijt me hr falpetrige Säure vor: handen, als juläifïg. Tritt bie blaue Färbung nur fđamad) und Iangiam ein, jo ijt bie \$rüfung zu wiederbolen und gleidyzeitig ein Sontrollveriuch) anzuiftellen, um bie nod) zuläfifige (Srenze Der Bläuung $z_{\mathfrak{u}}$ beftimmen. $3 \mathfrak{u}$ biejem 3 wed mijobt man in einem

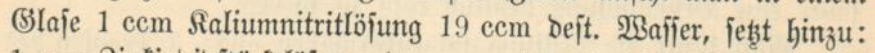
$1 \mathrm{ccm}$ Zinfjobiojtärfelöjung, $1 / 2 \mathrm{ccm}$ verdünnte Schmefeliäure. $3 u$ Derjelben Beit wiro in einem andern SIaje bie Nija)ung zu 2

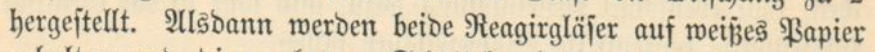
gehalten und bie nach Dem Schütteln eintretende Farbenverände= rung beobachtet. Sit ber in ber Rontrollflüffigfeit entitandene Farbenton heller als in ber zu unterjuchenden Flüiffitgfeit, jo ijt

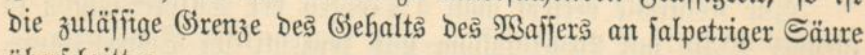
überichritten.

\section{Interjuduแn aนf $\mathfrak{A m m o n i a f . ~}$}

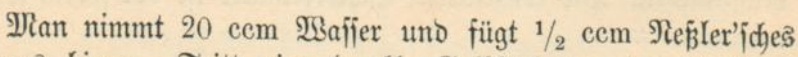
Reagens bingu. Tritt eine Dunfle Gelbfärbung oder Rötbung 
oder gar ein rother Nieberichlag ein, fo ift $\mathfrak{A m m o n i a f}$ in zu reids= licker Menge vorbanden.

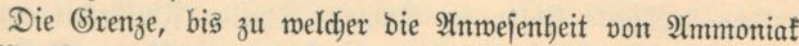
zuläffitg ifft, ermittelt man folgendermaj̃en: man bemahrt bie \$robe auf, erzeugt fich in einem zweiten (SIlaje mit $1 \mathrm{ccm}$ ber in $\Re$ r. 10 Der Reagentien erwähnten Flüffitgfeit, welche mit $19 \mathrm{ccm}$ Dejt.

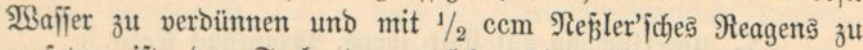
verieben ift, Den Farbenton, melcher die gröfïte noch zuläifige Nenge $\mathfrak{2}$ mmoniaf anzeigt. Sit Der Farbenton ber zu unter= fudjenden Flüffïgfeit Dunfler als berjenige Der Sontrollflüifïgfeit, fo ijt bie zuläfifige (Srenze überjofritten. ${ }^{1}$ )

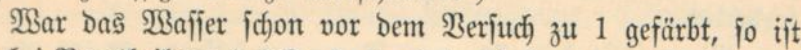
Dies bei Beurtheilung Des Farbentons in Rect)mung zu jtellen und barf in biejem Falle nur bei Entitehung eines rothen Farbentons die S'egenmart von 2 (mmmoniaf angenommen werden.

Jit das झुaffer ftarf falf= bezw. magnefiabaltig, fo wiro

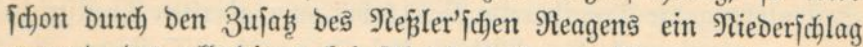
erzeugt, Der allerbings bei গtbmejenbeit von $\mathfrak{A m m o n i a f}$ und in farblojem $\mathfrak{B a f j e r}$ weip ijt.

Sind mur Spuren von 2Ammoniaf vorbanden und ift baร

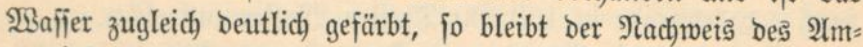

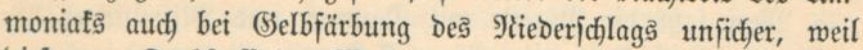

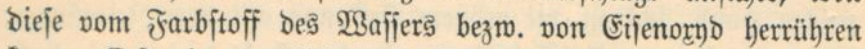

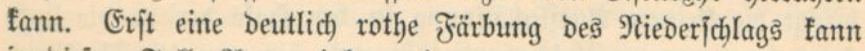
in biejem Falle Itmmoniaf anzeigen.

\section{Itnterjudumg anj (6hlor.}

$20 \mathrm{ccm}$ ßafjer merben mit Salpeterjäure angejäuert uno mit etrwa 10 Iropfen Silbernitratlöjung verjeşt. Entjteht feine Fäl=

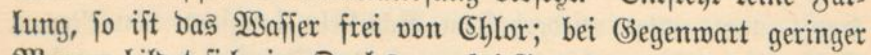
Mengen bil'Det fïch eine Dpalešcenz, bei (Segenwart größ̄erer Mengen eine beutlicje weipe Trübung bezw. Albịbeioung weiper Flodfen.

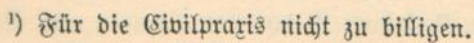


Bur Schäbung, ob ber (Srenzmerth erreidft ift ober nicht, bient bie unter 4 ber Reagentien angegebene Sialiumdbloriolöjung, welche 3 Theile હhlor in 100,000 Theilen Flüfirgteit enthält. $20 \mathrm{ccm}$ biejer \&öjung werben wie bas zu prüfende Waffer be= handelt und bie entitandenen Trübungen mit einander verglidgen.

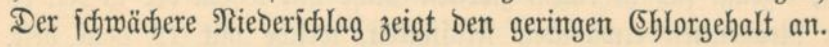

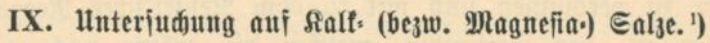

Beim Mangel an Beit genügt bie Bejtimmung ber $(S)=$ jammtbärte, jonjt ijt audb) mit Rüdjidbt auf bie Sdjmefel= fäureunterjudyung bie Bejtimmung ber bleibenden 5 ärte er= forberlich.

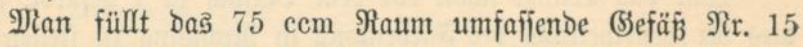
ber (Seräthe bis zur Marfe $(40 \mathrm{ccm})$ und ftellt es verftöpjelt bei Seite.

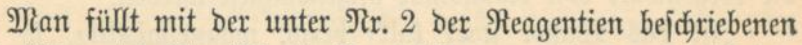
Seifenlöjung bie Iropfbürette $\mathfrak{N}$ ir. 16 bis zum oberjten Theiljtric, indem man einen fpişigen (Ślaştab in bie größ̧ere Deffinung ein= jentt und an bemjelben bie Seifenlöjung langjam heruntergleiten läb̄t (ber Innenraum ber Bürette ift jo grabuirt, baj jeder Theil= (tric) 1 ફ̧ärtegrabe in 100,000 Theilen $\mathfrak{W a j f e r ~ e n t i p r i c h t ) . ~}$

Man fap̧t bie Bürette mit Daumen uno Mittelfinger ber

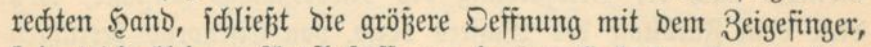

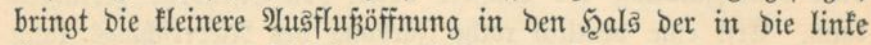

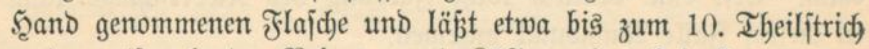
unter entiprectjender Neigung uno \&üftung Desె Beigefingers von ber Seifenlöjung einfliejen. Mian verftöpjelt mummebr bie Flajdje wieber und fdüuttelt ftarf mit auf= und abrwärtsgebenden $\mathfrak{B e}=$ wegungen.

3eigt fich nach Dem S(fjütteln fein feinblajiger S(j)aum, jo tropft man unter wieberboltem Schütteln langam von Neuem Seifenlöjung hinzu, biş burd) Den leb̧ten Iropfen ber gemünj(d)te zarte, 5 Minuten lang ftehende Schaum erzeugt wirb.

J) Das vorgeidriebene $\mathfrak{B e r f a h r e n ~ i j t ~ b a s ~ v o n ~ B o u t r o n ~ u n b ~ B o u b e t . ~}$ 
Die 3abl, weldbe bem Stand ber Seifenlöjung bei jentrechter Scaltung ber Bürette entipriçt, zeigt unmittelbar bie franzöjijichen

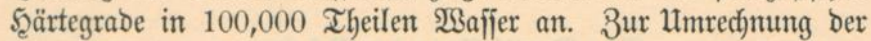
franzöpijidyen in beutiche Şärtegrabe multiplizirt man bie 3ahl ber eriteren mit 0,56 .

Sollten mebr als 22 Srade Seifenlöĭung biş zur Schaum= biloung erforberlich jein, jo muß ber ßerjud wieberholt werben, weil fich) über 22 binaus Feblerquellen ergeben. Man verbünnt

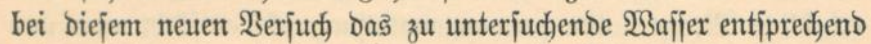
und multiplizirt mit Dem ßerbünnungsfoëffizienten (z. $\mathfrak{B}$. ange= wandt find $10 \mathrm{ccm}$ Wajjer, zu $40 \mathrm{ccm}$ mit bejtillirtem $\mathfrak{\text { Bafjer }}$ veroünnt, verbraucht find 10 Srabe Seifenlöjung, Sejammtbärte $=10 \cdot 4=40$ ).

Die Beftimmung Der bleibenden 5 ärte hat injofern eine gewiffie Bebeutung für bie Beurtheilung bes Wafjers, als mitteljt

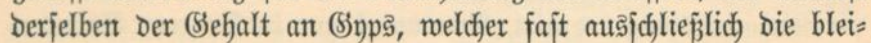
bende Scärte bebingt, und Der (Sehalt an Sd)mefeljäure, meldje

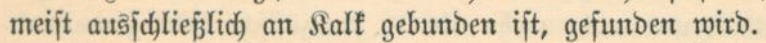

Behufs ber Ulnterjuct)ung füllt man $100 \mathrm{ccm}$ Wafjer in ben

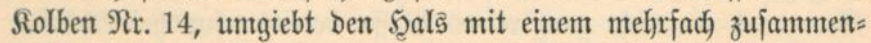
gefalteten \$apierítreifen, erfaß̧t Den überitehenoen Flügel befijelben und fodjt $1 / 2$ Stunde Iang über ber Spirituslampe. Das ver= Dampfende $\mathfrak{W a f f e r ~ m u p ~ D a b e i ~ v o n ~ 3 e i t ~ z u ~ B e i t ~ b u r d ) ~ D e j t i l l i r t e ß ~}$ Waffer exjeb̨t merben. Nach bem Rochen filtrirt man jobnell, läp̈t erfalten, füllt zu $100 \mathrm{ccm}$ auf und beftimmt in $40 \mathrm{ccm}$ bie ફ̧ärte wie oben.

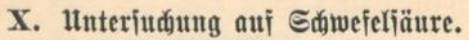

Die Beftimmung ber bleibenden ફ̧ärte fann für bie Sçäłung ber vorbandenen Menge Schwefeljäure, ba eine quantitative (5e= midjtsanalyje im felbe nicht auşü̈hrbar ijt, benubzt merden. Man wiro feine allzu grop̄en F̌bler begehen, menn man bie gejammte Sdjmefeljäure als an Ralf gebunden annimmt unb bemtgemäp auf 


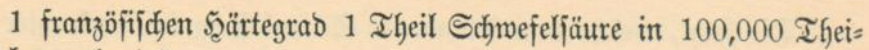
Ien rechnet. ${ }^{1}$ )

\section{Beurtheilung Der Rejultate.}

Ein Wajier, meldhes jonjt gute Eigenidhaften hat und auf IImmoniaf, Salpeter $=$ und jalpetrige Säure j(fwact) reagirt, fann nod) als braudbbar bezeidfnet merben.

Irïbe $\mathfrak{W a j f e r ~ f i n t o ~ f t e t ̦ ~ v e r b a ̈ d f t i g , ~ n a m e n t l i c ̧ ) , ~ w e n n ~ e i n e ~}$ erhebliche Menge אaliumpermanganatlöjung rajoch entfärbt wiro, uno bie Reaftionen auf $\mathfrak{A}$ mmoniaf, falpetrige und Salpeterjäure beutlid, hervortreten. Derartige Waffer Dürfen jebenfalls nur gereinigt oder filtritt genofjen werben.

श्Ils rein und gejund wirb nach ber gemöhnlichen 2 Innahme

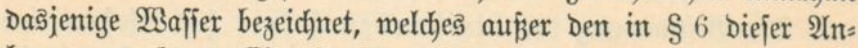
lage angegebenen (Figenjidaften nicht mehr als 1 I heil Salium: permanganatlöjung reduzirt, Immmoniaf nur in Spuren, Salpeter= fäure mur in Den angegebenen (Srenzen, falpetrige Säure aber gar nicht entbält und nicht mebr als 36. franzöjifiche Şärtegrade hat. Dagegen ift nicht mit Sicherheit allgemein bejtimmbar, von welcher

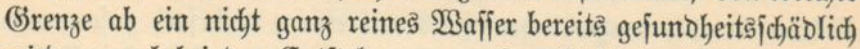
wirb, zumal bei ber Entitehung von Siranfheiten veridjiedene $1 \mathrm{tr}=$ jachen einmirfen fönnen.

1) Der zuläfifige Grentziverth Der Sdimefelfäure in Dem Irinfwaijer $(0,05$ bis 0,06 im siter) ift nididt angegebent. 


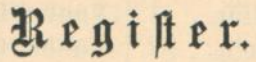

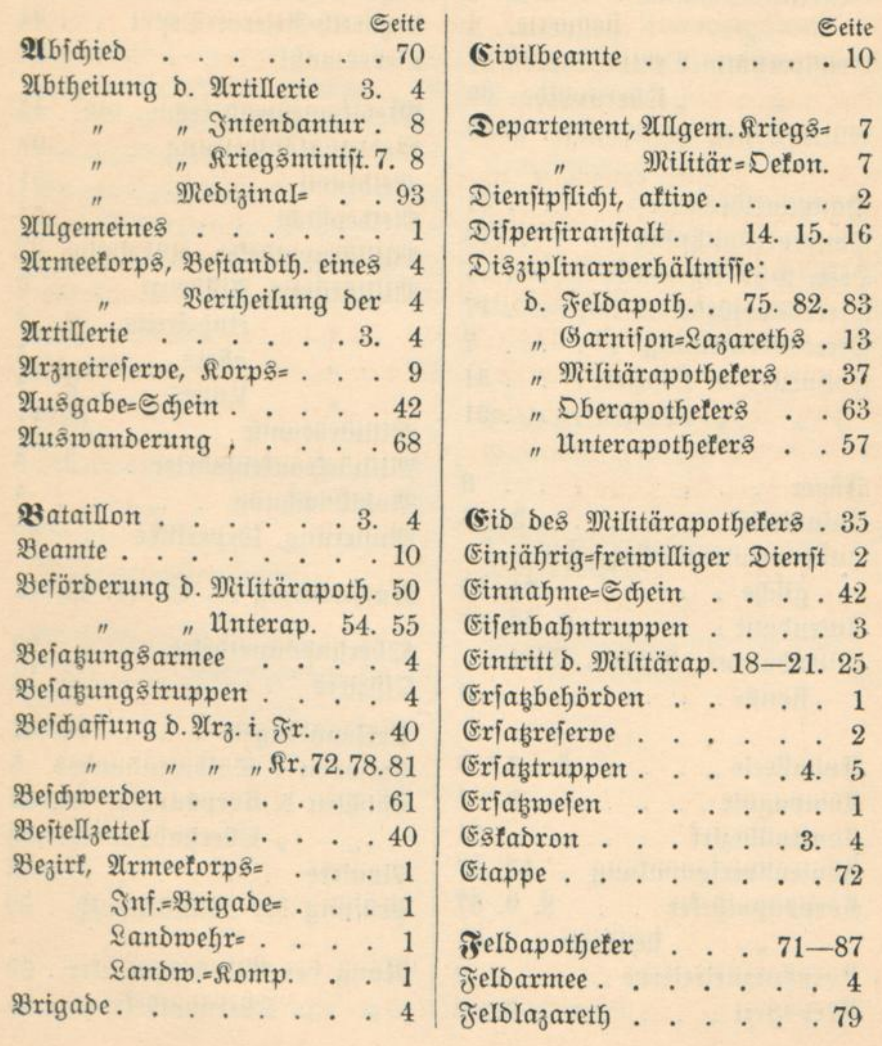




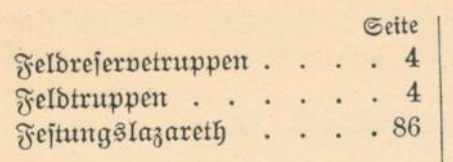

(Garnifonlazareth) . . . 13 Gebieţeintfeiluntg, militär. . 1 Gebührnifie ठ. Stabsapoth. . 87 "Dberjtabsap.. 93 Generaltommanoo . . . 4. 8 jtellvertr. 4

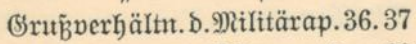
" "Dberapoth. . 68 Siuter=Depot, inmobiles. . 85

Sauptmeldeamt . . . . . 51 ङauptverbantoplak . . . 76 5eer, Das eigentlidje . . . 1 5eereşapothefer . . . . . 17 5eeresperwaltung . . . . 7 5ouneutrs ธ. Dffiziere. . . 31 $"$ " ïbrigen . . . 31

รäger . . . . . . . . 3 ฐnfanterie . . . . . . 3. 5 Эnitrumentenmad)er, d)irur $=$ gifide. . . . 84.85 ฐntendant . . . . 8. 36.37 Эntenbantux, Rorpss=, Divi= fions: 8

Savallerie. . . 3. 4. 5 Rompagnie . . . . . 3. 4 Rontrollbezirt . . . . . . 51 Rontrollveriammliung . 52.57 §orpşapotheter . . 8. 9.87 ftellvertr. . . 91 Rorpsarzneirejerve. . . 9 Rorpsarzt...... 8.9
Seite

Rriegsılazareth) . . . . 83 Rriegsిminijterium . . . 7.8 Sriegs 3 ujtanঠ . . . . . 4

Qanojturmpflidyt. . . . . 2

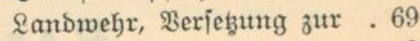
Sandwebrbienjtpflid)t . . . 2 \&anbwefritamunrolle . . . 51 \&a arethgehülfe . . . . 6 $\mathcal{L} \mathfrak{a}_{3}$ areth $=$ Rejerve $=$ Depot . . 84 ¿iebesmahl . . . . 67

Medifamentenbered)n., tał . . 45 Mesizinalabtyeilumg . . . 93 Melbeamt . . . . . . . 51 Meldepflidft . . . . . . 51 Militärapothełer, eint..Freim. 32

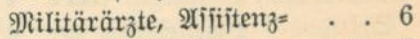

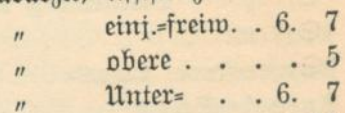
Militärbeamte . . . 10. 11 Mitlitärfranfenwärter . . . 5. 6 Mobilmadjung . . . . . 4 Mutjterung, förperfid)e . . 26 Radjweifung 40. 43. 44. 45.48 Dberitabsapothefer . . . 93 Dffizizere . . . . . 5. 12 \$erionalbogen . . . 55. 67 \$erjonen D. Soldatenitandes 5 \$flidften D. Rorpsapoth. 88. 89 " "Dberítabsapoth. 93 \$ioniere . . . . . . . 3 \$rïfung ber Militärapoth. . 50

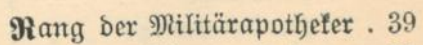
"Dberapotbeter. . 55 
Eeite

Rangoromung für Miltiär= beamte . . . 10.11 Red)mung

Redjnungslegung . . . . . 33 Regiment . . . . . . 4 Requifition . . . . . . 72

Rejervedienjtpflidft . . . . 2 Rejerve $=$ Divifition . . . . 4 Rejerve $=\left\{\mathfrak{a}_{3}\right.$ areth . . . 86

๔anitäts=Detact)ement. . . .74 Sanitätø๖bienjt imt Frieben .13 $"$ im Sriege 73. 74. 77. 81 Sanitätsłorps . . . . . 5 Santiätsof offizizere . . 5. 6. 12 Sanitätß̊perjonen . . . . 5 Sdjriftwerfehr mit $\mathfrak{B}$ ehörben 22 Seftionen ๖. Senteraltonm. . 8 Soldaterifand . . . . 5 Stabsapothefer . . . . . 87

Train . . . . . . . 3

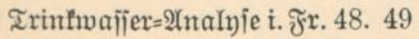
Iruppentfeile . . . . . 3 Iruppenverbantoplakg . . . 74 utniform ๖. 2tuditeure. $\quad 36.37$ " " Jnten๖anten 36. 37

\section{Seite}

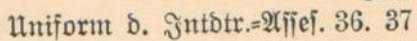
" = Rätbe 36.37 " " Лorps= u. Ober= roß̉ärz̧te 36.37 Militärapoth. . . 32 Dberapothefer . 56 "Sanitätsoffiziziere 5 " Sanitätsperion. 5 "Stabsapotheter. 88 "Unterapotheter. 51

" " Zahlmeiter 36.37 Unterapothefer . . . . . 51 Unterofifiziere . . . . . . 5 Unteriutudungen, chemifid)e .91 " Des Trintwaīers im Frieden 94-102

Unterrid)t Der $\mathfrak{M}$ ilitiärapoth. 50

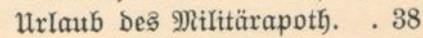
" "Stabsapoth. . 88

Berbanbmittelrejerve . . . 9

Berwaltunģ̧Dienjt . . . . 13

$\mathfrak{3 a f f e n g a t t u n g e n ~ . ~ . ~ . ~ . ~} 3$

\$Befrpflidft. . . . . . . 1

3urü̈đfitellung hinter Die leķte

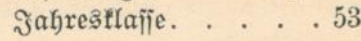




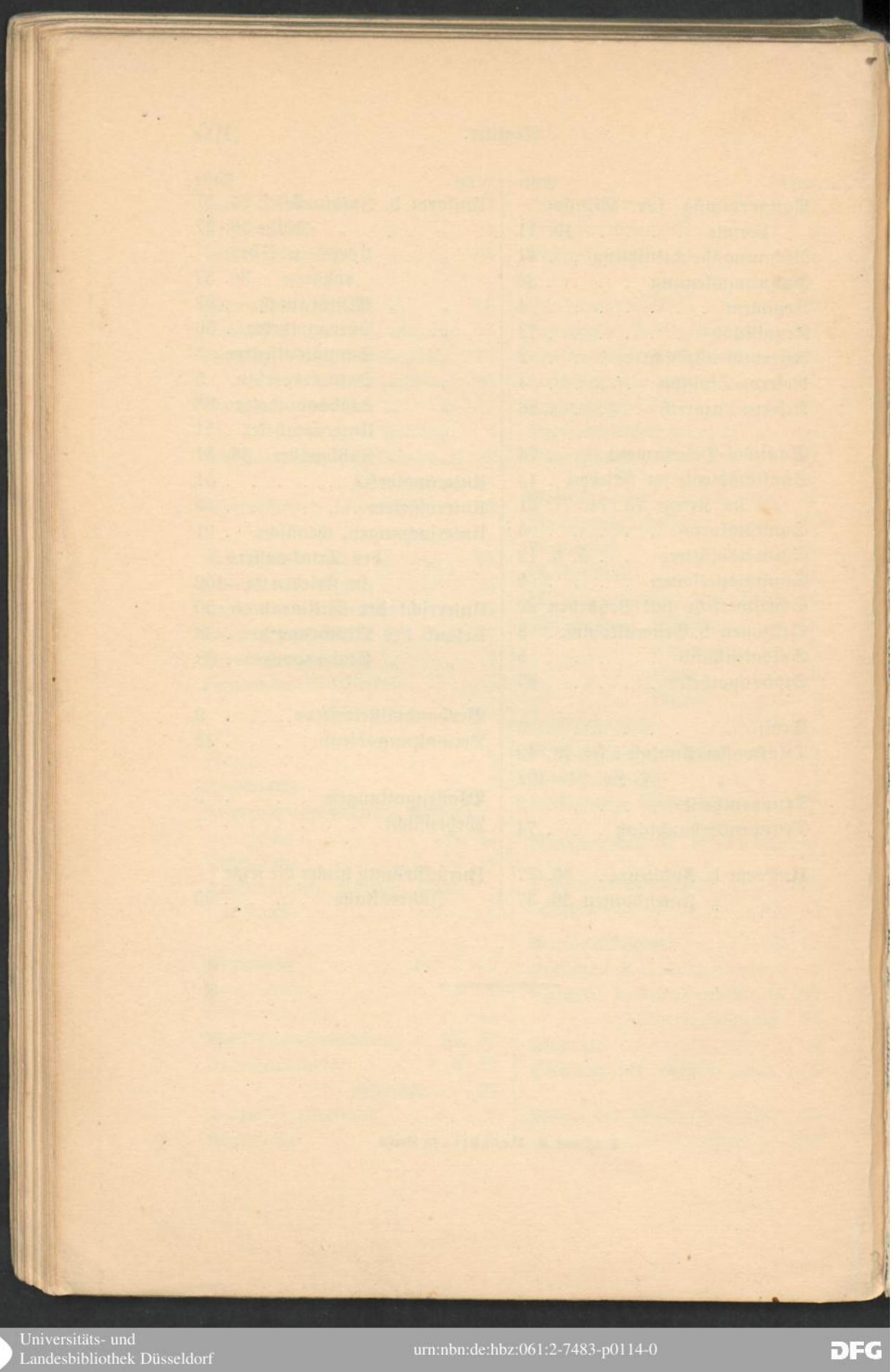


Berlag von Sulius Springer in Berlin.

\title{
Die Apotheken-Gesetzgebung
}

\author{
des
}

đeutschen Reiches und der Einzelstaaten

anf der Grundlage der allgemeinen politischen, Handels- und Gewerbegesetzgebung dargestellt.

Herausgegeben und mit ausfuhrlichen Erläuterungen versehen von

Dr. H. Böttger.

Redakteur der Pharmaceutischen Zeitung.

2 Bïnde,

I. Bd.: Reichsgesetzgebung. - II. Bd.: Landesgesetzgebung.

C. Preis jedes Bandes M. 7,-; gebunden M. 8,-.

$\therefore \quad$ Die gesetzlichen Bestimmungen

uber die $\rightarrow$ :

Rechte und Pflichteh der Deutschen Apotheker-Gehülfen.

Zusammengestellt

voin

Dr. H. Böttger,

Redakteur der Pharmaceutischen Zeitunk.

Preis kart. M. 1,40 .

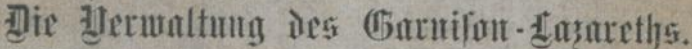

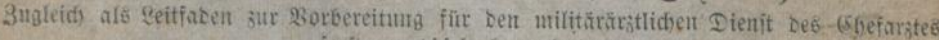
ibfeuatiods bearbeftet

voit

Di. (5. Flas'sar,

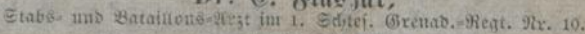

fint. Areis a: 1.60.

\section{Pharmaceutischer Kalender.}

Herausgegeben von

- Dr. H. Böttger und Dr. B. Fischer. I. Theil: Tages-, Notiz-, Blüthen-, Sammel-, Arbeits-Kaleuder, nebst Regeln und
Hiilfsmittehn firr praktische Pharmacie.

II. Theil: Pharmaceutisches Jahrbuch.

I: Theil geb. in Leimwand.

II. Theil geheftet.

Preis zusammen M. 3,-.
I. Theil geb. in Leder.

II. Theil geheftet.

Preis zusammen M. 3,50 .

3u bejieben oura jede Budbandung. 